\title{
The effect of projections on dimension in the Heisenberg group
}

\author{
Zoltán M. Balogh, Estibalitz Durand-Cartagena, Katrin Fässler, \\ Pertti Mattila and Jeremy T. Tyson
}

\begin{abstract}
We prove analogs of classical almost sure dimension theorems for Euclidean projection mappings in the first Heisenberg group, equipped with a sub-Riemannian metric.
\end{abstract}

\section{Introduction}

In this paper, we study projection mappings from the Heisenberg group onto horizontal lines and complementary vertical planes. In particular we consider the effect of such mappings on the Hausdorff dimensions and Hausdorff measure of subsets of the Heisenberg group considered with respect to a sub-Riemannian metric.

Our results are analogs, in sub-Riemannian geometry, for classical theorems of Marstrand [16]. We shall employ potential theoretic methods first used in this context by Kaufman in [13] and later generalized in [17]. There have been many studies of Marstrand type projection results. For example, a general Fourier analytic machinery for projection-type theorems was developed by Peres and Schlag in [21]. See also the survey [19] for an overview of the subject.

This paper represents part of an extensive program aimed at extending geometric measure theory beyond the Euclidean setting, the origins of which date back to Gromov's groundbreaking treatise [11].

The Heisenberg group $\mathbb{H}$ is the unique analytic nilpotent Lie group whose background manifold is $\mathbb{R}^{3}$ and whose Lie algebra $\mathfrak{h}$ admits a vector space decomposition $\mathfrak{h}=\mathfrak{v}_{1} \oplus \mathfrak{v}_{2}$, where $\mathfrak{v}_{1}$ has dimension two, $\mathfrak{v}_{2}$ has dimension one, and the Lie bracket identities $\left[\mathfrak{v}_{1}, \mathfrak{v}_{1}\right]=\mathfrak{v}_{2}$ and $\left[\mathfrak{h}, \mathfrak{v}_{2}\right]=0$ hold.

We identify $\mathbb{H}$ with $\mathbb{C} \times \mathbb{R}=\mathbb{R}^{3}$ through exponential coordinates. Points in $\mathbb{H}$ are denoted $p=(z, t)$. We work throughout this paper with the following convention for the group law:

$$
(z, t) *(\zeta, \tau)=(z+\zeta, t+\tau+2 \operatorname{Im}(z \bar{\zeta})) .
$$

Mathematics Subject Classification (2010): 28A78, 28A80, 43A80.

Keywords: Heisenberg group, projection theorems, Hausdorff dimension, energy integrals. 
Our results are formulated with respect to a sub-Riemannian structure on the Heisenberg group. We will work primarily with the well known Heisenberg metric on $\mathbb{H}$ (also known as the Korányi metric). This is the left invariant metric given by

$$
d_{\mathbb{H}}(p, q)=\left\|q^{-1} * p\right\|_{\mathbb{H}},
$$

where $\|\cdot\|_{\mathbb{H}}$ is the gauge norm defined by

$$
\|p\|_{\mathbb{H}}=\left(|z|^{4}+t^{2}\right)^{1 / 4} .
$$

Note that $d_{\mathbb{H}}$ is bi-Lipschitz equivalent to the Carnot-Carathéodory metric on $\mathbb{H}$ which can be defined using horizontal curves. An absolutely continuous curve $\gamma: I \rightarrow \mathbb{H} \simeq \mathbb{R}^{3}$ on an interval $I$ in $\mathbb{R}$ is called horizontal if

$$
\dot{\gamma}(s) \in H_{\gamma(s)} \mathbb{H} \quad \text { for almost every } s \in I,
$$

where $H_{p} \mathbb{H}=\operatorname{span}\left\{X_{p}, Y_{p}\right\}$ with $X=\partial_{x}+2 y \partial_{t}$ and $Y=\partial_{y}-2 x \partial_{t}$.

All results which we shall obtain regarding Hausdorff dimensions of subsets of $\mathbb{H}$ are unchanged under bi-Lipschitz change of the metric. The advantage of working with the metric $d_{\mathbb{H}}$, rather than using the Carnot-Carathéodory metric, is its simple and explicit form.

There is also a one-parameter family of nonisotropic dilation mappings $\left(\delta_{r}\right)_{r>0}$ given by

$$
\delta_{r}(z, t)=\left(r z, r^{2} t\right)
$$

We recall that the Hausdorff dimension of the metric space $\left(\mathbb{H}, d_{\mathbb{H}}\right)$ is equal to 4 . In fact, $\left(\mathbb{H}, d_{\mathbb{H}}\right)$ is an Ahlfors 4-regular metric space.

The Heisenberg group $\mathbb{H}$ has the structure of an $\mathbb{R}$ bundle over the plane $\mathbb{R}^{2}$. We write $\pi: \mathbb{H} \rightarrow \mathbb{R}^{2}$ for the mapping

$$
\pi(z, t)=z
$$

and note that $\pi$ is 1 -Lipschitz as a map from $\left(\mathbb{H}, d_{\mathbb{H}}\right)$ to $\left(\mathbb{R}^{2}, d_{E}\right)$. Here and throughout this paper, $d_{E}$ denotes the Euclidean metric on any Euclidean space.

A subgroup $G$ of $\mathbb{H}$ is called a homogeneous subgroup if it is invariant under the dilation semigroup $\left(\delta_{r}\right)_{r>0}$, i.e.,

$$
p \in G, r>0 \quad \Longrightarrow \quad \delta_{r}(p) \in G .
$$

Observe that, under the aforementioned identification of $\mathbb{H}$ with $\mathbb{R}^{3}$, homogeneous subgroups of $\mathbb{H}$ are vector subspaces of $\mathbb{R}^{3}$. For fixed $\theta \in[0, \pi)$, let $\mathbb{V}_{\theta}$ be the one-dimensional subspace of $\mathbb{R}^{3}$ spanned by the vector $\left(e^{\mathbf{i} \theta}, 0\right)$. Then $\mathbb{V}_{\theta}$ is a homogeneous subgroup of $\mathbb{H}$. Let $\mathbb{W}_{\theta}$ be the Euclidean orthogonal complement of $\mathbb{V}_{\theta}$, i.e., the two-dimensional subspace of $\mathbb{R}^{3}$ spanned by the vectors $\left(\mathbf{i} e^{\mathbf{i} \theta}, 0\right)$ and $(0,1)$. Then $\mathbb{W}_{\theta}$ is also a homogeneous subgroup of $\mathbb{H}$. We will identify $\mathbb{V}_{\theta}$ with $\mathbb{R}$ via the global chart

$$
\left(r e^{\mathrm{i} \theta}, 0\right) \stackrel{\varphi \mathbb{V}_{\theta}}{\longmapsto} r
$$

and we will identify $\mathbb{W}_{\theta}$ with $\mathbb{R}^{2}$ via the global chart

$$
\left(a \mathbf{i} e^{\mathbf{i} \theta}, t\right) \stackrel{\varphi_{\mathbb{W}}}{\longmapsto}(a, t) .
$$


In this paper, we call the homogeneous subgroups $\mathbb{V}_{\theta}, \theta \in[0, \pi)$, horizontal subgroups and we call the subgroups $\mathbb{W}_{\theta}, \theta \in[0, \pi)$, vertical subgroups. Both types of subgroups are abelian subgroups of $\mathbb{H}$, in addition, vertical subgroups are normal subgroups of $\mathbb{H}$. Note also that the restriction of $d_{\mathbb{H}}$ to a horizontal subgroup $\mathbb{V}_{\theta}$ coincides with the restriction of the Euclidean metric of $\mathbb{R}^{3}$ to $\mathbb{V}_{\theta}$. We therefore may speak about metric properties of the horizontal subgroups $\mathbb{V}_{\theta}$ without reference to the metric. On the other hand, the restriction of $d_{\mathbb{H}}$ to a vertical subgroup $\mathbb{W}_{\theta}$ is given by

$$
d_{\mathbb{H}}\left(\varphi_{\mathbb{W}_{\theta}}^{-1}(a, t), \varphi_{\mathbb{W}_{\theta}}^{-1}\left(a^{\prime}, t^{\prime}\right)\right)=\left(\left(a-a^{\prime}\right)^{4}+\left(t-t^{\prime}\right)^{2}\right)^{1 / 4}
$$

and is comparable to the parabolic (heat) metric $\left|a-a^{\prime}\right|+\left|t-t^{\prime}\right|^{1 / 2}$ on $\mathbb{R}^{2}$.

For each parameter $\theta$, the pair $\mathbb{V}_{\theta}$ and $\mathbb{W}_{\theta}$ induces a semidirect group splitting $\mathbb{H}=\mathbb{W}_{\theta} * \mathbb{V}_{\theta}$. For $p \in \mathbb{H}$, we write

$$
p=p_{\mathbb{W}_{\theta}} * p_{\mathbb{V}_{\theta}},
$$

where $p_{\mathbb{W}_{\theta}} \in \mathbb{W}_{\theta}$ and $p_{\mathbb{V}_{\theta}} \in \mathbb{V}_{\theta}$. In this way, we define the horizontal projection $p_{\mathbb{V}_{\theta}}: \mathbb{H} \rightarrow \mathbb{V}_{\theta}$ and vertical projection $p_{\mathbb{W}_{\theta}}: \mathbb{H} \rightarrow \mathbb{W}_{\theta}$ by the formulas

$$
p_{\mathbb{V}_{\theta}}(p)=p_{\mathbb{V}_{\theta}}
$$

and

$$
p_{\mathbb{W}_{\theta}}(p)=p_{\mathbb{W}_{\theta}}
$$

Explicit expressions for these mappings appear in (2.6) and (2.7). The semidirect splitting of $\mathbb{H}$ (and more general Carnot groups) into horizontal and vertical subgroups has played a key role in recent developments concerning intrinsic subRiemannian submanifold geometry and sub-Riemannian geometric measure theory, see for example [10], [9], [15], and [20].

The mappings $p_{\mathbb{V}_{\theta}}$ and $p_{\mathbb{W}_{\theta}}$ have rather different characters. The horizontal projection maps $p_{\mathbb{V}_{\theta}}$ are linear projection maps with respect to the underlying Euclidean structure on $\mathbb{R}^{3}$. Moreover, they are also Lipschitz maps (with Lipschitz constant 1) and homogeneous group homomorphisms of $\mathbb{H}$. On the other hand, the vertical projection mappings $p_{\mathbb{W}_{\theta}}$ are neither linear, nor (Euclidean) projections, nor group homomorphisms. These facts highlight the difficulty of working with the vertical projection mappings in the Heisenberg group. Nevertheless, we will ultimately be able to derive estimates for the effect of vertical projection on the Hausdorff dimensions of sets.

We denote by dim the Hausdorff dimension in a general metric space, and by $\mathcal{H}^{s}, s>0$, the corresponding family of Hausdorff measures. By $\mathcal{H}_{\delta}^{s}, \delta>0$, we denote the Hausdorff premeasures in dimension $s$. We will work with these notions for both the Heisenberg and Euclidean metrics $d_{\mathbb{H}}$ and $d_{E}$ on $\mathbb{H} \simeq \mathbb{R}^{3}$, so we will take care to specify the metric with which we are working, writing $\mathcal{H}_{\mathbb{H}}^{s}, \mathcal{H}_{E}^{s}$ and $\operatorname{dim}_{\mathbb{H}}, \operatorname{dim}_{E}$. Similarly we will denote by $B_{E}(p, r)$, respectively $B_{\mathbb{H}}(p, r)$, the ball of radius $r$ and center $p$ in the metric space $\left(\mathbb{R}^{3}, d_{E}\right)$, respectively $\left(\mathbb{H}, d_{\mathbb{H}}\right)$. We emphasize that we always consider closed balls in this paper. 
Our main theorems provide universal and almost sure estimates for the (Heisenberg) dimensions of horizontal and vertical projections of Borel subsets of $\mathbb{H}$. By a universal estimate we mean an inequality relating either $\operatorname{dim}_{\mathbb{H}} p_{\mathbb{V}_{\theta}}(A)$ or $\operatorname{dim}_{\mathbb{H}} p_{\mathbb{W}_{\theta}}(A)$ to $\operatorname{dim}_{\mathbb{H}} A$ which is valid for all sets $A$ and all angles $\theta$. By an almost sure estimate we mean an inequality relating these quantities which is valid for all sets $A$ and for $\mathcal{L}^{1}$-almost every angle $\theta$. Henceforth all measure theoretic statements involving the angle $\theta$ will be made with respect to the Lebesgue measure $\mathcal{L}^{1}$.

Let $A \subset \mathbb{H}$ be a Borel set. Since the horizontal projection maps are Lipschitz and the horizontal subspaces are one-dimensional, the estimate

$$
\operatorname{dim} p_{\mathbb{V}_{\theta}}(A) \leq \min \left\{1, \operatorname{dim}_{\mathbb{H}} A\right\}
$$

holds for all $\theta$. Note that the dimension of $p_{\mathbb{V}_{\theta}}(A)$ with respect to the Heisenberg metric is the same as with respect to the Euclidean distance. We first state which universal and almost sure lower bounds hold for the dimensions of horizontal projections.

Theorem 1.1 (Universal lower bounds for horizontal projections). Let $A$ be a Borel set in $\mathbb{H}$. Then

$$
\operatorname{dim} p_{\mathbb{V}_{\theta}}(A) \geq \max \left\{0, \operatorname{dim}_{\mathbb{H}} A-3\right\} \quad \text { for all } \theta .
$$

The estimate in (1.6) is sharp.

Theorem 1.2 (Almost sure lower bounds for horizontal projections). Let $A \subset \mathbb{H}$ be a Borel set. Then

$$
\operatorname{dim} p_{\mathbb{V}_{\theta}}(A) \geq \max \left\{0, \min \left\{\operatorname{dim}_{\mathbb{H}} A-2,1\right\}\right\} \quad \text { for a.e. } \theta .
$$

If $\operatorname{dim}_{\mathbb{H}} A>3$, then $\mathcal{H}^{1}\left(p_{\mathbb{V}_{\theta}}(A)\right)>0$ for a.e. $\theta$. The estimate in (1.7) is sharp.

Figure 1 illustrates the sets of universal and almost sure dimension pairs for horizontal projections on $\mathbb{H}$.
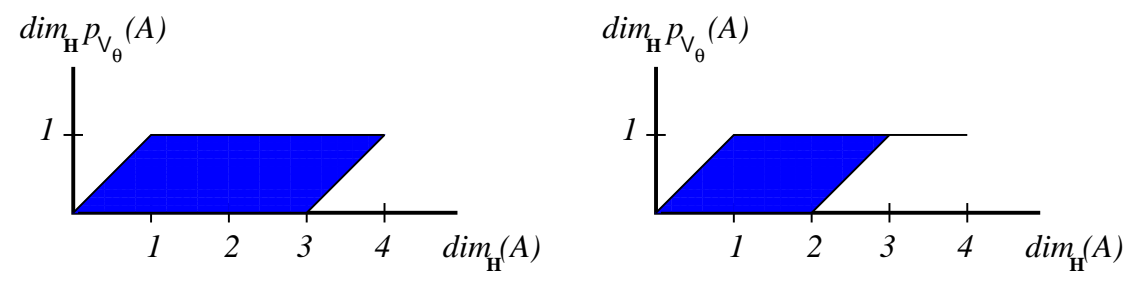

FiguRE 1. (a) Universal dimension pairs for horizontal projections; (b) almost sure dimension pairs for horizontal projections

The proof of Theorem 1.2 is rather straightforward. It uses simple estimates for the dimension of the projection $\pi(A)$ combined with classical almost sure dimension theorems for Euclidean projections. The sharpness parts of Theorems 1.2 and 1.1 are contained in Proposition 3.2. 
The state of our knowledge regarding the effect of the vertical projections on Hausdorff dimension is less advanced. However, we are able to obtain some results. Namely, we can show the following theorems. Note that the Hausdorff dimension of the vertical subgroups $\mathbb{W}_{\theta}$ with respect to the Heisenberg metric $d_{\mathbb{H}}$ on $\mathbb{H}$ is equal to 3 .

Theorem 1.3 (Universal upper and lower bounds for vertical projections). Let $A \subset \mathbb{H}$ be a Borel set. Then

$$
\operatorname{dim}_{\mathbb{H}} p_{\mathbb{W}_{\theta}}(A) \leq \min \left\{2 \operatorname{dim}_{\mathbb{H}} A, \frac{1}{2}\left(\operatorname{dim}_{\mathbb{H}} A+3\right), 3\right\} \quad \text { for all } \theta,
$$

and

$$
\operatorname{dim}_{\mathbb{H}} p_{\mathbb{W}_{\theta}}(A) \geq \max \left\{0, \frac{1}{2}\left(\operatorname{dim}_{\mathbb{H}} A-1\right), 2 \operatorname{dim}_{\mathbb{H}} A-5\right\} \quad \text { for all } \theta .
$$

The universal estimates in (1.8) and (1.9) are sharp.

Theorem 1.4 (Almost sure lower bounds for vertical projections). Let $A$ be a Borel set in $\mathbb{H}$. If $\operatorname{dim}_{\mathbb{H}} A \leq 1$, then

$$
\operatorname{dim}_{\mathbb{H}} p_{\mathbb{W}_{\theta}}(A) \geq \operatorname{dim}_{\mathbb{H}} A \text { for a.e. } \theta .
$$

Consequently, for any $A$,

$$
\operatorname{dim}_{\mathbb{H}} p_{\mathbb{W}_{\theta}}(A) \geq \max \left\{\min \left\{\operatorname{dim}_{\mathbb{H}} A, 1\right\}, 2 \operatorname{dim}_{\mathbb{H}} A-5\right\} \quad \text { for a.e. } \theta .
$$

The estimate (1.11) is sharp when $\operatorname{dim}_{\mathbb{H}} A \leq 1$.
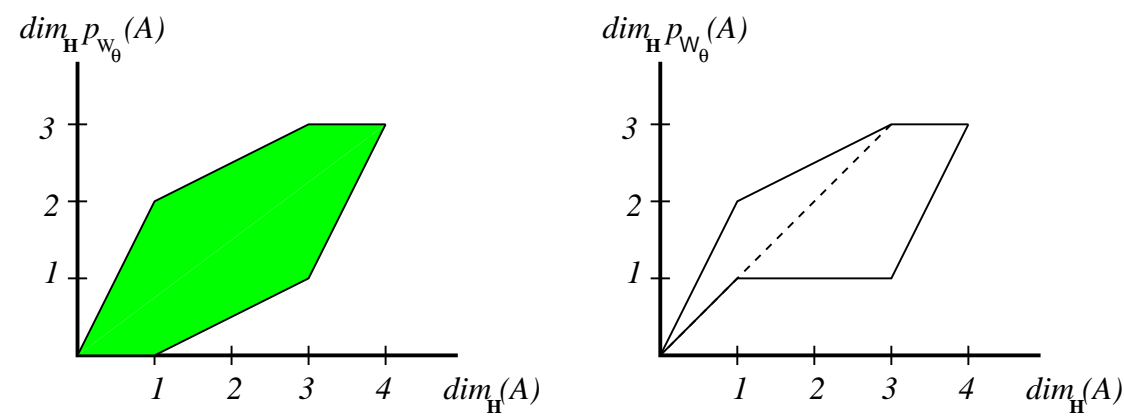

FiguRE 2. (a) Universal dimension pairs for vertical projections; (b) almost sure dimension pairs for vertical projections (including conjectured sharp lower bound)

The sharpness statement of Theorem 1.3 is discussed in Proposition 4.10. The upper bound (1.8) is also sharp as an almost sure statement, see Proposition 5.3. Examples which prove the sharpness of the lower bound (1.11) in Theorem 1.4 in the case when $\operatorname{dim}_{\mathbb{H}} A \leq 1$ are given by subsets of the $t$-axis. We do not know whether the lower bound (1.11) is sharp in the case when $1<\operatorname{dim}_{\mathbb{H}} A<4$ but we suspect not. We formulate the following:

Conjecture 1.5. For all $A \subset \mathbb{H}$, $\operatorname{dim}_{\mathbb{H}} p_{\mathbb{W}_{\theta}}(A) \geq \min \left\{\operatorname{dim}_{\mathbb{H}} A, 3\right\}$ for a.e. $\theta$. If $\operatorname{dim}_{\mathbb{H}} A>3$, then $\mathcal{H}_{\mathbb{H}}^{3}\left(p_{\mathbb{W}_{\theta}}(A)\right)>0$ for a.e. $\theta$. 
Proposition 6.1 and Theorem 6.3 provide partial evidence in support of Conjecture 1.5.

Figure 2 illustrates the sets of universal and almost sure dimension pairs for vertical projections on $\mathbb{H}$ (including the conjectured sharp lower bound).

The lower bounds in Theorem 1.4 can be improved in case the set $A$ is a subset of either a horizontal plane or a vertical plane. See Section 7 for details.

We would like to emphasize an important difference between Theorems 1.2 and 1.4 and their Euclidean predecessor, see Theorem 2.3 below. Namely, for any Borel set $A \subset \mathbb{R}^{n}$, the almost sure dimension of the image $P_{V}(A)$ under a Euclidean projection on an $m$-dimensional subspace $V$ can be computed exactly as a function of $\operatorname{dim}_{E} A$ and $m$. No similar formula holds in the Heisenberg setting, at least for arbitrary Borel sets. Indeed, the best result which can be obtained is a pair of (distinct) upper and lower bounds for the Heisenberg dimensions of the projections. We give a variety of examples to demonstrate the sharpness of our estimates. Finally, let us remark that we do obtain an exact formula for the $\mathcal{L}^{1}$-almost sure dimension of the horizontal projection in the low codimensional case $\operatorname{dim}_{\mathbb{H}} A>3$. Conjecturally, a similar exact formula holds for the vertical projections under the same assumption on $\operatorname{dim}_{\mathbb{H}} A$.

We conclude this introduction with an outline of the paper. In Section 2 we recall preliminary information concerning almost sure dimension theorems in Euclidean space and the dimension comparison principle in the Heisenberg group. Section 3 treats the case of the horizontal projection mappings and contains the proof of Theorems 1.1 and 1.2. Section 4 contains the proof of the universal dimension bounds for the vertical projection mappings, Theorem 1.3. The main results of the paper concerning the almost sure dimension theorem for vertical projections, Theorem 1.4 and the related examples, are presented in Section 5. Since our results on almost sure dimensions of vertical projections are rather incomplete, we will discuss several classes of examples where we have a better understanding of the behavior of the dimension of the projections. The first such class consists of sets with a certain degree of regularity. This is discussed in Section 6. In Section 7 we sharpen the analysis of the vertical projections, obtaining improved dimension estimates for projections of subsets of horizontal or vertical planes. Section 8 contains remarks and open questions motivated by this study.

Acknowledgements. Research for this paper was initiated while EDC and JTT were guests in the Mathematics Institute of the University of Bern in Fall 2009, and completed while PM was a guest of the same institute in Fall 2010. The hospitality of the institute is gratefully appreciated.

\section{Review of background material}

\subsection{Dimension and Euclidean projections}

Theorems 1.2 and 1.4 are adaptations to the Heisenberg setting of classical almost sure dimension theorems for Euclidean projections, proved by Marstrand in the plane [16] and generalized in [17]. We briefly recall the Euclidean theorems. 
Definition 2.1. Let $m$ and $n$ be integers with $0<m<n$. The Grassmanian $G(n, m)$ is the space of all $m$-dimensional linear subspaces of $\mathbb{R}^{n}$.

It is possible to introduce a natural measure $\gamma_{n, m}$ on $G(n, m)$. In the case $m=1$ this measure is fairly simple to describe. In fact, the Grassmanian $G(n, 1)$ coincides with the real projective space $P_{\mathbb{R}}^{n-1}$, and the measure in question is the pushforward of the surface measure from $\mathbb{S}^{n-1}$ under the canonical quotient map $\mathbb{S}^{n-1} \rightarrow P_{\mathbb{R}}^{n-1}$. For instance, $G(2,1)$ can be identified with $P_{\mathbb{R}}^{1}$, or even more explicitly with the interval $\left[0, \pi\right.$ ) (by identifying a line through the origin in $\mathbb{R}^{2}$ with the angle $\theta \in[0, \pi)$ which it makes with the positive $x$-axis). Under the latter identification, the measure in question is just $\mathrm{d} \theta$. Via the canonical identification of the Grassmanians $G(n, m)$ and $G(n, n-m)$, we could also describe the natural measure on $G(n, n-1)$ quite explicitly. However, for $2 \leq m \leq n-2$ the story is more complicated. We refer to Chapter 3 of [18] for the construction of the measure $\gamma_{n, m}$ on $G(n, m)$. It can be checked that $\gamma_{n, m}$ is equivariant with respect to the usual action of the orthogonal group $O(n)$ on $G(n, m)$.

Remark 2.2. The measure $\gamma_{n, m}$ can be constructed in another manner. The Grassmanian $G(n, m)$ is a smooth manifold of dimension $m(n-m)$, and is also a metric space when equipped with the function $d(V, W)=\left\|P_{V}-P_{W}\right\|$. Here $P_{V}: \mathbb{R}^{n} \rightarrow V$ denotes orthogonal projection from $\mathbb{R}^{n}$ onto a subspace $V$, and $\|\cdot\|$ denotes the operator norm. Up to a multiplicative constant, the measure $\gamma_{n, m}$ coincides with the Hausdorff measure $\mathcal{H}^{m(n-m)}$ on the metric space $(G(n, m), d)$. This follows easily from the fact that both of the measures in question are $O(n)$ equivariant, and hence uniformly distributed. See Definition 3.3 and Theorem 3.4 in [18] for additional details.

Theorem 2.3 (Euclidean Projection Theorem). Let $m$ and $n$ be integers with $0<$ $m<n$ and let $A \subset \mathbb{R}^{n}$ be a Borel set. If $\operatorname{dim}_{E} A \leq m$, then $\operatorname{dim}_{E} P_{V}(A)=\operatorname{dim}_{E} A$ for $\gamma_{n, m}$-a.e. $V \in G(n, m)$. If $\operatorname{dim}_{E} A>m$, then $\mathcal{H}^{m}\left(P_{V}(A)\right)>0$ for $\gamma_{n, m}$-a.e. $V \in G(n, m)$. In particular,

$$
\operatorname{dim}_{E} P_{V}(A)=\min \left\{\operatorname{dim}_{E} A, m\right\} \quad \text { for } \gamma_{n, m} \text {-a.e. } V .
$$

A Suslin set is the continuous image of a Borel set. Theorem 2.3 extends to Suslin sets.

Frostman's lemma is a standard tool used in the proof of lower bounds for Hausdorff dimension. We denote by $\mathcal{M}(A)$ the collection of positive, finite Borel regular measures supported on a set $A$ of a metric space $X$.

Theorem 2.4 (Frostman's lemma). Let A be a Borel (Suslin) subset of a complete metric space $(X, d)$. Suppose that there exist $s>0, \mu \in \mathcal{M}(A)$, and $r_{0} \in(0, \infty]$ so that the inequality

$$
\mu(B(x, r)) \leq r^{s}
$$

holds for all $x \in A$ and $0<r<r_{0}$. Then $\mathcal{H}^{s}(A)>0$. In particular, $\operatorname{dim} A \geq s$.

Conversely, if $\mathcal{H}^{s}(A)>0$ then there exists a measure $\mu \in \mathcal{M}(A)$ so that $(2.2)$ holds for all $x \in A$ and $r>0$. 
See, e.g., Proposition 4.2 in [8], [12], or Theorem 8.17 in [18].

We say that $\mu$ satisfies an upper mass bound on $A$ with exponent $s$ if (2.2) holds for all $x \in A$ and $0<r<r_{0}$.

Next, we state the energy version of Frostman's lemma. This follows easily from Theorem 2.4; see Chapter 8 of [18].

Definition 2.5. Let $(X, d)$ be a metric space and let $\mu \in \mathcal{M}(X)$. For $s>0$, the $s$-energy of $\mu$ is

$$
I_{s}(\mu)=\int_{X} \int_{X} d(x, y)^{-s} \mathrm{~d} \mu(x) \mathrm{d} \mu(y) .
$$

Theorem 2.6 (Energy version of Frostman's lemma). Let A be a Borel (Suslin) subset of a complete metric space $(X, d)$ and let $s>0$ be such that there exists $\mu \in \mathcal{M}(A)$ with $I_{s}(\mu)<\infty$. Then $\operatorname{dim} A \geq s$. Conversely, if $A$ is a Borel (Suslin) subset of a complete metric space $(X, d)$ and $s<\operatorname{dim} A$, then there exists $\mu \in \mathcal{M}(A)$ with $I_{s}(\mu)<\infty$.

\subsection{Dimension comparison principle}

We will make use of the recent solution to the dimension comparison problem in $\mathbb{H}$. This problem, originally posed by Gromov in Section 0.6.C of [11], asks for sharp estimates relating the Euclidean and Heisenberg measures and dimensions of subsets of $\mathbb{H}$. A nearly complete answer was given by Balogh, Rickly and SerraCassano [4]; the story was completed by Balogh and Tyson [5], who gave examples demonstrating the sharpness of the lower bound. We state the final result, in its sharp form.

Theorem 2.7 (Dimension comparison in the Heisenberg group). Let $A \subset \mathbb{H}$ be a set with $\operatorname{dim}_{E} A=\alpha \in[0,3]$ and $\operatorname{dim}_{\mathbb{H}} A=\beta \in[0,4]$. Then

$$
\max \{\alpha, 2 \alpha-2\}=: \beta_{-}(\alpha) \leq \beta \leq \beta_{+}(\alpha):=\min \{2 \alpha, \alpha+1\} .
$$

Moreover, for any pair $(\alpha, \beta) \in[0,3] \times[0,4]$ satisfying $\beta_{-}(\alpha) \leq \beta \leq \beta_{+}(\alpha)$, there is a compact set $A_{\alpha, \beta} \subset \mathbb{H}$ with $\operatorname{dim}_{E} A_{\alpha, \beta}=\alpha$ and $\operatorname{dim}_{\mathbb{H}} A_{\alpha, \beta}=\beta$.

Theorem 2.7 was generalized to arbitrary Carnot groups by Balogh, Tyson and Warhurst [6].

From now on, we refer to the estimates in (2.3) as the dimension comparison principle for the Heisenberg group $\mathbb{H}$.

We will also use the dimension comparison principle in vertical subgroups of $\mathbb{H}$. Due to the special form (1.4) of the restriction of the Korányi metric to such subspaces, we obtain stronger dimension comparison estimates therein. To wit, we have

Theorem 2.8 (Dimension comparison in vertical subgroups of the Heisenberg group). Let $A \subset \mathbb{W}_{\theta}$ be a set contained in some vertical subgroup $\mathbb{W}_{\theta} \subset \mathbb{H}$, with $\operatorname{dim}_{E} A=\alpha \in[0,2]$ and $\operatorname{dim}_{\mathbb{H}} A=\beta \in[0,3]$. Then

$$
\max \{\alpha, 2 \alpha-1\}=: \beta_{-}^{\mathbb{W}}(\alpha) \leq \beta \leq \beta_{+}^{\mathbb{W}}(\alpha):=\min \{2 \alpha, \alpha+1\} .
$$

Theorem 2.8 can be proved by adapting the arguments from [6]. 


\subsection{Explicit formulas for horizontal and vertical projections}

We present explicit formulas for the projection mappings $p_{\mathbb{V}_{\theta}}$ and $p_{\mathbb{W}_{\theta}}$, and for the distance between points in $\mathbb{H}$ and the corresponding distance between their projections. Such formulas will be useful in the proofs of Theorems 1.2 and 1.4.

Let $\theta \in[0, \pi)$ and let $p=(z, t) \in \mathbb{H}$. We recall that the projections $p_{\mathbb{V}_{\theta}}$ and $p_{\mathbb{W}_{\theta}}$ are determined by the identity

$$
p=p_{\mathbb{W}_{\theta}} * p_{\mathbb{V}_{\theta}} .
$$

The horizontal projection $p_{\mathbb{V}_{\theta}}$ coincides with the Euclidean orthogonal projection $P_{\mathbb{V}_{\theta}}: \mathbb{R}^{3} \rightarrow \mathbb{V}_{\theta}$ and is given by

$$
p_{\mathbb{V}_{\theta}}(z, t)=p_{\mathbb{V}_{\theta}}=\left(\operatorname{Re}\left(e^{-\mathbf{i} \theta} z\right) e^{\mathbf{i} \theta}, 0\right) .
$$

The vertical projection $p_{\mathbb{W}_{\theta}}$ can then be determined via (2.5) and is given by

$$
p_{\mathbb{W}_{\theta}}(z, t)=p_{\mathbb{W}_{\theta}}=\left(\operatorname{Im}\left(e^{-\mathbf{i} \theta} z\right) \mathbf{i} e^{\mathbf{i} \theta}, t-\operatorname{Im}\left(e^{-2 \mathbf{i} \theta} z^{2}\right)\right) .
$$

Denote by $p=(z, t)$ and $q=(\zeta, \tau)$ two points in $\mathbb{H}$. Observing that

$$
\operatorname{Im}((z-\zeta)(\overline{z+\zeta}))=2 \operatorname{Im}(z \bar{\zeta})
$$

and using the formula (1.1) for the group law in $\mathbb{H}$, we record the following expression for the distance between $p$ and $q$ :

$$
d_{\mathbb{H}}^{4}(p, q)=\left\|q^{-1} * p\right\|_{\mathbb{H}}^{4}=|z-\zeta|^{4}+\left(t-\tau+\left|z^{2}-\zeta^{2}\right| \sin \left(\varphi_{1}-\varphi_{2}\right)\right)^{2} .
$$

Here we write $\varphi_{1}=\arg (z-\zeta)$ and $\varphi_{2}=\arg (z+\zeta)$.

Similarly, the distance between $p_{\mathbb{V}_{\theta}}(p)$ and $p_{\mathbb{V}_{\theta}}(q)$ can be expressed in the form

$$
d_{\mathbb{H}}\left(p_{\mathbb{V}_{\theta}}(p), p_{\mathbb{V}_{\theta}}(q)\right)=\left\|p_{\mathbb{V}_{\theta}}(q)^{-1} * p_{\mathbb{V}_{\theta}}(p)\right\|_{\mathbb{H}}=|z-\zeta|\left|\cos \left(\varphi_{1}-\theta\right)\right| .
$$

Finally, the distance between $p_{\mathbb{W}_{\theta}}(p)$ and $p_{\mathbb{W}_{\theta}}(q)$ can be expressed in the form

$$
\begin{aligned}
& d_{\mathbb{H}}^{4}\left(p_{\mathbb{W}_{\theta}}(p) p_{\mathbb{W}_{\theta}}(q)\right)=\left\|p_{\mathbb{W}_{\theta}}(q)^{-1} * p_{\mathbb{W}_{\theta}}(p)\right\|_{\mathbb{H}}^{4} \\
& =|z-\zeta|^{4} \sin ^{4}\left(\varphi_{1}-\theta\right)+\left(t-\tau-\left|z^{2}-\zeta^{2}\right| \sin \left(\varphi_{2}+\varphi_{1}-2 \theta\right)\right)^{2} .
\end{aligned}
$$

Note that the vertical projections $p_{\mathbb{W}_{\theta}}: \mathbb{H} \rightarrow \mathbb{W}_{\theta}$ are locally $\frac{1}{2}$-Hölder continuous with respect to the Heisenberg metric. This is an easy computation involving the explicit formula for the projection.

\section{Projections onto horizontal subspaces}

In this section, we discuss the effect of horizontal projections on Borel sets in the Heisenberg group.

We begin with a lemma on the relationship between the Heisenberg dimension of a set in $\mathbb{H}$ and the Euclidean dimension of its planar projection.

Lemma 3.1. For any set $A \subset \mathbb{H}$, we have $\operatorname{dim}_{E} \pi(A) \geq \operatorname{dim}_{\mathbb{H}} A-2$. 
Proof. We may assume without loss of generality that $A$ is bounded. In fact, let us assume that $|t| \leq 1$ for all points $p=(z, t) \in A$.

Let $s>\operatorname{dim}_{E} \pi(A)$, let $\varepsilon>0$, and cover the set $\pi(A)$ with a family of Euclidean balls $\left\{B_{E}\left(z_{i}, r_{i}\right)\right\}_{i}$ so that $\sum_{i} r_{i}^{s}<\varepsilon$. Since $\pi:\left(\mathbb{H}, d_{\mathbb{H}}\right) \rightarrow\left(\mathbb{R}^{2}, d_{E}\right)$ is 1-Lipschitz, the fiber $\pi^{-1}\left(B_{E}(z, r)\right)$ contains the ball $B_{\mathbb{H}}((z, t), r)$ for any $t \in \mathbb{R}$. We can choose an absolute constant $C_{0}>0$ and $N_{i} \leq C_{0} r_{i}^{-2}$ values $t_{i j}$ so that the family $\left\{B_{\mathbb{H}}\left(\left(z_{i}, t_{i j}\right), C_{0} r_{i}\right)\right\}_{j}$ covers the set $B_{E}\left(z_{i}, r_{i}\right) \times[-1,1]$. Then $\left\{B_{\mathbb{H}}\left(\left(z_{i}, t_{i j}\right), C_{0} r_{i}\right)\right\}_{i, j}$ covers the set $A$. Denoting by $\operatorname{rad}(B)$ the radius of a ball $B$, we compute

$$
\sum_{i, j} \operatorname{rad}\left(B_{\mathbb{H}}\left(\left(z_{i}, t_{i j}\right), C_{0} r_{i}\right)\right)^{s+2}=\sum_{i} N_{i}\left(C_{0} r_{i}\right)^{s+2} \leq C_{0}^{s+3} \sum_{i} r_{i}^{s} \leq C_{0}^{s+3} \varepsilon .
$$

Letting $\varepsilon \rightarrow 0$ gives $\mathcal{H}_{\mathbb{H}}^{s+2}(A)=0$ so $\operatorname{dim}_{\mathbb{H}} A \leq s+2$. Letting $s \rightarrow \operatorname{dim}_{E} \pi(A)$ completes the proof.

Proof of Theorems 1.1 and 1.2. Let $A \subset \mathbb{H}$ satisfy $\operatorname{dim}_{\mathbb{H}} A>2$. By Lemma 3.1, $\operatorname{dim}_{E} \pi(A) \geq \operatorname{dim}_{\mathbb{H}} A-2$. Let us identify the one-dimensional subspace of $\mathbb{R}^{2}$ spanned by the vector $e^{\mathbf{i} \theta}$ with the corresponding one-dimensional subspace $\mathbb{V}_{\theta} \subset \mathbb{H}$. This allows us to consider the Euclidean projection map $P_{\mathbb{V}_{\theta}}$ as a (1-Lipschitz) map from $\mathbb{R}^{2}$ to $\mathbb{V}_{\theta}$.

Applying the Euclidean Projection Theorem 2.3 to $\pi(A)$ (note that $\pi(A)$ is a Suslin set) and noting that $p_{\mathbb{V}_{\theta}}=P_{\mathbb{V}_{\theta}} \circ \pi$, we find

$$
\operatorname{dim} p_{\mathbb{V}_{\theta}}(A)=\operatorname{dim}_{E} P_{\mathbb{V}_{\theta}}(\pi(A)) \geq \min \left\{1, \operatorname{dim}_{E} \pi(A)\right\} \geq \min \left\{1, \operatorname{dim}_{\mathbb{H}} A-2\right\}
$$

for a.e. $\theta$. This proves (1.7). In the case when $\operatorname{dim}_{\mathbb{H}} A>3$, we use the second part of Theorem 2.3 to arrive at the desired conclusion $\mathcal{H}^{1}\left(p_{\mathbb{V}_{\theta}}(A)\right)>0$ for a.e. $\theta$. Finally, for any $\theta$, we have

$$
\operatorname{dim}_{\mathbb{H}} p_{\mathbb{V}_{\theta}} A=\operatorname{dim}_{E} P_{\mathbb{V}_{\theta}}(\pi(A)) \geq \operatorname{dim}_{E} \pi(A)-1 \geq \operatorname{dim}_{\mathbb{H}} A-3 .
$$

The proof is complete.

Both the universal bounds and the almost sure bounds for dimension distortion by horizontal projections are sharp. We collect relevant examples demonstrating this in the following proposition.

Proposition 3.2. In each of the following statements, the set $A$ is a compact subset of $\mathbb{H}$ :

(a) For all $0 \leq \beta \leq 1$ there exists $A$ so that

$$
\operatorname{dim}_{\mathbb{H}} A=\beta \quad \text { and } \quad \operatorname{dim} p_{\mathbb{V}_{\theta}}(A)=\beta \text { for all } \theta .
$$

(b) For all $1 \leq \beta \leq 4$ there exists $A$ so that

$$
\operatorname{dim}_{\mathbb{H}} A=\beta \quad \text { and } \quad \operatorname{dim} p_{\mathbb{V}_{\theta}}(A)=1 \text { for all } \theta .
$$

(c) For all $0 \leq \beta \leq 3$ there exists $A$ so that

$$
\operatorname{dim}_{\mathbb{H}} A=\beta \quad \text { and } \quad \operatorname{dim} p_{\mathbb{V}_{0}}(A)=0 .
$$

If $0 \leq \beta \leq 2$ we can choose $A$ so that $\operatorname{dim} p_{\mathbb{V}_{\theta}}(A)=0$ for all $\theta$. 
(d) For all $3 \leq \beta \leq 4$ there exists $A$ so that

$$
\operatorname{dim}_{\mathbb{H}} A=\beta \quad \text { and } \quad \operatorname{dim} p_{\mathbb{V}_{0}}(A)=\beta-3 .
$$

(e) For all $2 \leq \beta \leq 3$ there exists $A$ so that

$$
\operatorname{dim}_{\mathbb{H}} A=\beta \quad \text { and } \quad \operatorname{dim} p_{\mathbb{V}_{\theta}}(A)=\beta-2 \text { for all } \theta .
$$

Recall that a Borel set $E$ is called an s-set, $s \geq 0$, if $0<\mathcal{H}^{s}(E)<\infty$. A bounded metric space $(X, d)$ is said to be Ahlfors regular of dimension $s \geq 0$ if there exists a measure $\mu \in \mathcal{M}(X)$ and a constant $C \geq 1$ so that

$$
C^{-1} r^{s} \leq \mu(B(x, r)) \leq C r^{s}
$$

for all $x \in X$ and $0<r<\operatorname{diam} X$. If $(X, d)$ is Ahlfors regular of dimension $s$, then $\operatorname{dim} X=s$ and $\mu$ is comparable to the Hausdorff measure $\mathcal{H}^{s}$.

Proof. For part (a), let $A_{0} \subset \mathbb{V}_{0}$ and $A_{\pi / 2} \subset \mathbb{V}_{\pi / 2}$ be compact $\beta$-sets. The set $A=A_{0} \cup A_{\pi / 2}$ verifies the stated conditions.

To show part (b) it suffices to construct a compact set $A$ with $\operatorname{dim}_{\mathbb{H}} A=\beta$ and such that $\pi(A)$ is a planar set which projects onto a one-dimensional subset of $\mathbb{V}_{\theta}$ for every $\theta$. We consider two cases. First, assume that $1 \leq \beta \leq 3$. Let $S \subset[0,1]$ be a compact $(\beta-1) / 2$-set and let $A=A_{0} \cup A_{\pi / 2}$, where $A_{0}=\{(x, t): 0 \leq x \leq 1$, $t \in S\}$ and $A_{\pi / 2}=\{(\mathbf{i} y, t): 0 \leq y \leq 1, t \in S\}$. Since the restriction of $d_{\mathbb{H}}$ to any vertical subgroup is comparable with the heat metric, $\operatorname{dim}_{\mathbb{H}} A=1+2 \operatorname{dim} S=\beta$. The set $\pi(A)$ is the union of two line segments which form a right angle at the origin. For $\theta=0$ and $\theta=\pi / 2$ one of the two segments is projected to a single point, but the projection of the entire set $\pi(A)$ on $\mathbb{V}_{\theta}$ is one-dimensional for every direction $\theta$ as desired. Next, assume that $3<\beta \leq 4$. In this case, take the set $A$ to be the union of any compact set of Heisenberg Hausdorff dimension $\beta$ with the set $\{(z, 0):|z| \leq 1\}$. This completes the proof for part (b).

We now turn to the proof of part (c). For the first claim, any compact $\beta$-set $A \subset \mathbb{W}_{0}$ suffices. In case $0 \leq \beta \leq 2$ we can choose this compact set $A$ to be a subset of the $t$-axis, in which case $p_{\mathbb{V}_{\theta}}(A)=\{(0,0)\}$ for all $\theta$.

Next we consider part (d). We may assume that $3<\beta<4$. Let $S \subset \mathbb{R}$ be a compact set which is Ahlfors regular of dimension $(\beta-3)$, let

$$
B_{0}=\{(\mathbf{i} y, t): 0 \leq y \leq 1,0 \leq t \leq 1\}
$$

and let

$$
A=\left\{p_{0} *(x, 0): p_{0} \in B_{0}, x \in S\right\} .
$$

Then $p_{\mathbb{V}_{0}}(A)=\{(x, 0): x \in S\}$ has dimension $\beta-3$. It suffices to prove that $\operatorname{dim}_{\mathbb{H}} A \geq \beta$. We will use Theorem 2.4. It suffices to show that the measure

$$
\mu(E):=\int_{S} \mathcal{H}_{\mathbb{H}}^{3}\left(\left\{p_{0} *(x, 0): p_{0} \in B_{0}\right\} \cap E\right) d \mathcal{H}_{E}^{\beta-3}(x)
$$

has the upper mass bound (2.2) on $A$ with exponent $\beta$. Let $B_{\mathbb{H}}(p, r)$ be a ball in $\left(\mathbb{H}, d_{\mathbb{H}}\right)$ centered at $p=p_{0} *\left(x_{0}, 0\right) \in A$ with radius $r$.

For $x \in S$, denote by $B_{x}$ the set of points of the form $q *(x, 0), q \in B_{0}$. 
Lemma 3.3. If $B_{\mathbb{H}}(p, r) \cap B_{x} \neq \emptyset$, then $\left|x-x_{0}\right| \leq r$ and

$$
\mathcal{H}_{\mathbb{H}}^{3}\left(B_{\mathbb{H}}(p, r) \cap B_{x}\right) \leq C r^{3}
$$

for a constant $C$ independent of $p, r$ and $x$.

Assuming the lemma we complete the proof in this case:

$$
\mu\left(B_{\mathbb{H}}(p, r)\right) \leq C r^{3} \cdot \mathcal{H}_{E}^{\beta-3}\left(\left[x_{0}-r, x_{0}+r\right] \cap A\right) \leq C^{\prime} r^{\beta} .
$$

Hence $\mu$ satisfies the upper mass bound (2.2) on $A$ with exponent $\beta$. By Theorem 2.4, $\operatorname{dim}_{\mathbb{H}} A \geq \beta$.

It remains to prove the lemma. Suppose that $B_{\mathbb{H}}(p, r) \cap B_{x} \neq \emptyset$. Then $B_{\mathbb{H}}(p, r) \subset B_{\mathbb{H}}(q, 2 r)$ for some $q \in B_{\mathbb{H}}(p, r) \cap B_{x}$ and

$$
\mathcal{H}_{\mathbb{H}}^{3}\left(B_{\mathbb{H}}(p, r) \cap B_{x}\right) \leq \mathcal{H}_{\mathbb{H}}^{3}\left(B_{\mathbb{H}}(q, 2 r) \cap B_{x}\right) \leq C r^{3},
$$

since $B_{x}$ lies in a vertical plane in $\mathbb{H}$. Furthermore, if $q=(\mathbf{i} y, t) *(x, 0)$ then $\left|x-x_{0}\right| \leq r$. This completes the proof of Lemma 3.3 and hence completes the construction for part (d).

Finally, we consider part (e). It suffices to construct a compact set $A \subset \mathbb{H}$ with $\operatorname{dim}_{\mathbb{H}} A=\beta$ such that $\pi(A)$ is a $(\beta-2)$-dimensional set in the plane whose dimension is preserved under $P_{\mathbb{V}_{\theta}}$ for every $\theta$. Let $S \subset \mathbb{R}$ be a compact $(\beta-2)$-set and let $A=A_{0} \cup A_{\pi / 2}$, where $A_{0}=\{(x, t): x \in S, 0 \leq t \leq 1\}$ and $A_{\pi / 2}=$ $\{(\mathbf{i} y, t): y \in S, 0 \leq t \leq 1\}$. Since the restriction of $d_{\mathbb{H}}$ to any vertical subgroup is comparable with the heat metric, we have that $\operatorname{dim}_{\mathbb{H}} A=\operatorname{dim} S+2=\beta$. Moreover, $\operatorname{dim}_{E} \pi(A)=\operatorname{dim}_{E} S=\beta-2$. The dimension of the set $\pi\left(A_{0}\right)$ is preserved under $P_{V_{\theta}}$ except for $\theta=\pi / 2$, in which case $\pi\left(A_{0}\right)$ is projected to a single point. An analogous statement holds for $\pi\left(A_{\pi / 2}\right)$ and the exceptional direction $\theta=0$. Altogether, it follows for all $\theta$ that $\operatorname{dim} p_{\mathbb{V}_{\theta}}(A)=\operatorname{dim} P_{\mathbb{V}_{\theta}}(\pi(A))=\beta-2$.

The proof of Proposition 3.2 is complete.

\section{Universal bounds for vertical projections}

In this section, we start to discuss the effect of vertical projections on the dimensions of Borel sets in the Heisenberg group. Our purpose is to prove Theorem 1.3.

We recall that the projection map $p_{\mathbb{W}_{\theta}}$ from $\mathbb{H}$ to the vertical subgroup $\mathbb{W}_{\theta}$ is given by

$$
p_{\mathbb{W}_{\theta}}(z, t)=\left(\operatorname{Im}\left(e^{-\mathbf{i} \theta} z\right) \mathbf{i} e^{\mathbf{i} \theta}, t-\operatorname{Im}\left(e^{-2 \mathbf{i} \theta} z^{2}\right)\right) .
$$

In contrast with the Euclidean case, this map is not Lipschitz continuous and hence does not a priori decrease dimension. Indeed, there are cases when this map increases dimension. Yet, there is still a certain control on the upper dimension bound coming from the local $\frac{1}{2}$-Hölder continuity of $p_{\mathbb{W}_{\theta}}$ with respect to $d_{\mathbb{H}}$. Thus, for an arbitrary subset $A$ of $\mathbb{H}$ and for all $\theta$, we have

$$
\operatorname{dim}_{\mathbb{H}} p_{\mathbb{W}_{\theta}}(A) \leq 2 \operatorname{dim}_{\mathbb{H}} A
$$


Example 4.1. Let $A=\{(1+\mathbf{i}) s, 0): s \in[0,1]\} \subset \mathbb{H}$ be a one-dimensional horizontal line segment. The image of $A$ under the projection $p_{\mathbb{W}_{0}}$ is the graph of a parabola contained in the vertical subspace $\mathbb{W}_{0}$. Thus $p_{\mathbb{W}_{0}}(A)$ is a non-horizontal smooth curve and so has Hausdorff dimension equal to two. This shows that for one-dimensional sets the upper bound (4.2) cannot be improved.

The proof of Theorem 1.3 is given in a series of propositions. Our first statement indicates the universal upper bounds which hold for the dimensions of vertical projections. Within a certain dimension range, the trivial upper bound given in (4.2) can be improved.

Proposition 4.2. Let $A \subset \mathbb{H}$ be any Borel set. Then, for every $\theta$,

$$
\operatorname{dim}_{\mathbb{H}} p_{\mathbb{W}_{\theta}}(A) \leq \min \left\{2 \operatorname{dim}_{\mathbb{H}} A, \frac{1}{2}\left(\operatorname{dim}_{\mathbb{H}} A+3\right), 3\right\}
$$

The cases $3<\operatorname{dim}_{\mathbb{H}} A \leq 4$ and $0 \leq \operatorname{dim}_{\mathbb{H}} A<1$ are trivial. The latter follows from the local $\frac{1}{2}$-Hölder continuity of $p_{\mathbb{W}_{\theta}}$. We will focus on the remaining case $1 \leq \operatorname{dim}_{\mathbb{H}} A \leq 3$. The proof in this situation is more involved and uses a covering argument.

Proposition 4.3. Let $A$ be a Borel subset of $\mathbb{H}$ with $\operatorname{dim}_{\mathbb{H}} A \in[1,3]$. For all $\theta \in[0, \pi)$ we have

$$
\operatorname{dim}_{\mathbb{H}} p_{\mathbb{W}_{\theta}}(A) \leq \frac{1}{2}\left(\operatorname{dim}_{\mathbb{H}} A+3\right) .
$$

The proof of this proposition is based on two preliminary results. Lemma 4.5, which describes the images of Heisenberg balls under vertical projections, and Lemma 4.6, which explains how this set can be covered efficiently by balls in the vertical plane. This allows us to find good covers for $p_{\mathbb{W}_{\theta}}(A)$, which then yields the desired upper bound for the Hausdorff dimension.

If not otherwise mentioned, we will in the following always identify the vertical plane $\mathbb{W}_{\theta}$ with $\mathbb{R}^{2}$ as described in (1.3). A point $p=\left(\alpha \mathbf{i} e^{\mathbf{i} \theta}, \tau\right)$ in $\mathbb{W}_{\theta}$ will be written in coordinates as $(\alpha, \tau)$.

Let $0<r<1$ and $x_{0} \in \mathbb{R}$. First, we describe the vertical projection in the direction $\theta \in[0, \pi)$ of a ball $B_{\mathbb{H}}\left(p_{0}, r\right)$ with center $p_{0}=\left(x_{0}, 0\right)$ on the $x$-axis. We prove that there is a "core curve" $\gamma_{\theta}^{x_{0}, r}$ such that the image of the ball under $p_{\mathbb{W}_{\theta}}$ lies in a small Euclidean neighborhood of the projected curve. For the following steps of the proof it will be essential to control the size of this neighborhood independently of the direction $\theta$. This can be achieved if one uses a different curve depending on whether $\theta$ is close to $\pi / 2$, or it is close to 0 or $\pi$.

Definition 4.4. The core curve $\gamma_{\theta}^{x_{0}, r}$ related to $x_{0} \in \mathbb{R}$ and $0<r<1$ is the subset of $\mathbb{H}$ given by

$$
\gamma_{\theta}^{x_{0}, r}:= \begin{cases}\left\{\left(x_{0}+\mathbf{i} y,-2 x_{0} y\right): y \in[-2 r, 2 r]\right\}, & \text { if } \theta \in\left[0, \frac{\pi}{4}\right] \cup\left[\frac{3 \pi}{4}, \pi\right), \\ \left\{\left(x_{0}+x, 0\right): x \in[-2 r, 2 r]\right\}, & \text { if } \theta \in\left(\frac{\pi}{4}, \frac{3 \pi}{4}\right) .\end{cases}
$$


A direct computation shows that for each $\theta \in[0, \pi), x_{0} \in \mathbb{R}$, and $0<r<1$, the image under $p_{\mathbb{W}_{\theta}}$ of the corresponding core curve $\gamma_{\theta}^{x_{0}, r}$ is the graph of a linear or quadratic function $f_{\theta}^{x_{0}, r}$ over an interval $I_{\theta}^{x_{0}, r}$.

Lemma 4.5. For all $\theta \in[0, \pi), p_{0}=\left(x_{0}, 0\right)$ with $x_{0} \in \mathbb{R}$, and $0<r<1$, we have

$$
p_{\mathbb{W}_{\theta}}\left(B_{\mathbb{H}}\left(p_{0}, r\right)\right) \subseteq N_{E}\left(p_{\mathbb{W}_{\theta}}\left(\gamma_{\theta}^{x_{0}, r}\right), 5 r^{2}\right),
$$

where the expression on the right denotes the Euclidean $5 r^{2}$-neighborhood of the set $p_{\mathbb{W}_{\theta}}\left(\gamma_{\theta}^{x_{0}, r}\right)$.

More precisely,

$$
\begin{aligned}
p_{\mathbb{W}_{\theta}}\left(B_{\mathbb{H}}\left(p_{0}, r\right)\right) & \\
& \subseteq\left\{(\alpha, \tau) \in \mathbb{R}^{2}: \alpha \in I_{\theta}^{x_{0}, r}, f_{\theta}^{x_{0}, r}(\alpha)-5 r^{2} \leq \tau \leq f_{\theta}^{x_{0}, r}(\alpha)+5 r^{2}\right\} .
\end{aligned}
$$

Proof of Lemma 4.5. We discuss the proof for the case $\theta \in(0, \pi / 4]$. The other cases can be treated similarly, using the appropriate core curve.

For an arbitrary point $\left(x^{\prime}+\mathbf{i} y^{\prime}, t^{\prime}\right)$ in the ball $B_{\mathbb{H}}\left(p_{0}, r\right)$, one finds

$$
\left|x^{\prime}-x_{0}\right| \leq r, \quad\left|y^{\prime}\right| \leq r \quad \text { and } \quad\left|t^{\prime}+2 x_{0} y^{\prime}\right| \leq r^{2}
$$

The projection is given by

$$
\begin{aligned}
p_{\mathbb{W}_{\theta}}\left(x^{\prime}+\mathbf{i} y^{\prime}, t^{\prime}\right) & =\left(-x^{\prime} \sin \theta+y^{\prime} \cos \theta, t^{\prime}+\left(x^{\prime 2}-y^{\prime 2}\right) \sin 2 \theta-2 x^{\prime} y^{\prime} \cos 2 \theta\right) \\
& =:\left(\alpha^{\prime}, \tau^{\prime}\right) .
\end{aligned}
$$

For points on the core curve, $\left(x_{0}+\mathbf{i} y,-2 x_{0} y\right) \in \gamma_{\theta}^{x_{0}, r}$, we have

$$
\begin{aligned}
p_{\mathbb{W}_{\theta}}\left(x_{0}+\mathbf{i} y-2 x_{0} y\right) & =\left(-x_{0} \sin \theta+y \cos \theta,-2 x_{0} y+\left(x_{0}^{2}-y^{2}\right) \sin 2 \theta-2 x_{0} y \cos 2 \theta\right) \\
& =:(\alpha, \tau) .
\end{aligned}
$$

Thus, as a subset of $\mathbb{R}^{2}$, the set $p_{\mathbb{W}_{\theta}}\left(\gamma_{\theta}^{x_{0}, r}\right)$ coincides with the graph of the function

$$
f_{\theta}^{x_{0}, r}(\alpha)=-2 \tan \theta\left(\alpha+\frac{x_{0}}{\sin \theta}\right)^{2}+x_{0}^{2}\left(\frac{2}{\sin \theta \cos \theta}-2 \tan \theta\right)
$$

over the interval

$$
I_{\theta}^{x_{0}, r}=\left[-x_{0} \sin \theta-2 r \cos \theta,-x_{0} \sin \theta+2 r \cos \theta\right] .
$$

The goal is now to find a point in $p_{\mathbb{W}_{\theta}}\left(\gamma_{\theta}^{x_{0}, r}\right)$ which lies close to the point $p_{\mathbb{W}_{\theta}}\left(x^{\prime}+\mathbf{i} y^{\prime}, t^{\prime}\right)$. To this end, let

$$
y:=y^{\prime}-\left(x^{\prime}-x_{0}\right) \tan \theta
$$

and note that

$$
|y| \leq\left|y^{\prime}\right|+\left|x^{\prime}-x_{0}\right||\tan \theta| \leq(1+|\tan \theta|) r \leq 2 r .
$$

It follows $\left(x_{0}+\mathbf{i} y,-2 x_{0} y\right) \in \gamma_{\theta}^{x_{0}, r}$. We claim that the Euclidean distance between the points $p_{\mathbb{W}_{\theta}}\left(x_{0}+\mathbf{i} y,-2 x_{0} y\right)$ and $p_{\mathbb{W}_{\theta}}\left(x^{\prime}+\mathbf{i} y^{\prime}, t^{\prime}\right)$ is at most $5 r^{2}$. 
First, we observe

$$
\begin{aligned}
\left|\alpha-\alpha^{\prime}\right| & =\left|\left(x^{\prime}-x_{0}\right) \sin \theta+\left(y-y^{\prime}\right) \cos \theta\right| \\
& =\left|\left(x^{\prime}-x_{0}\right) \sin \theta-\left(x^{\prime}-x_{0}\right) \tan \theta \cos \theta\right|=0
\end{aligned}
$$

for $y$ as in (4.8).

Second, we compute

$$
\left|\tau-\tau^{\prime}\right|=\left|-2 x_{0} y-t^{\prime}+\left(x_{0}^{2}-y^{2}-x^{2}+y^{\prime 2}\right) \sin 2 \theta-2 \cos 2 \theta\left(x_{0} y-x^{\prime} y^{\prime}\right)\right| .
$$

Inserting $y$ from (4.8) and using trigonometric relations yields

$$
\begin{aligned}
\left|\tau-\tau^{\prime}\right|= & \mid-2 x_{0} y^{\prime}-t^{\prime}+2 x_{0}\left(x^{\prime}-x_{0}\right) \tan \theta \\
& +2 \sin \theta \cos \theta\left(x_{0}^{2}+2 y^{\prime}\left(x^{\prime}-x_{0}\right) \tan \theta-\left(x^{\prime}-x_{0}\right)^{2} \tan ^{2} \theta-x^{2}\right) \\
& -2\left(\cos ^{2} \theta-\sin ^{2} \theta\right)\left(x_{0} y^{\prime}-x_{0}\left(x^{\prime}-x_{0}\right) \tan \theta-x^{\prime} y^{\prime}\right) \mid \\
= & \mid-\left(t^{\prime}+2 x_{0} y^{\prime}\right)+2 x_{0}\left(x^{\prime}-x_{0}\right) \sin \theta \cos \theta+2 x^{\prime}\left(x_{0}-x^{\prime}\right) \sin \theta \cos \theta \\
& -2 y^{\prime}\left(x_{0}-x^{\prime}\right)-2 \frac{\sin ^{3} \theta}{\cos \theta}\left(x^{\prime}-x_{0}\right)^{2} \mid \\
= & \left|-\left(t^{\prime}+2 x_{0} y^{\prime}\right)-2\left(x_{0}-x^{\prime}\right)^{2} \sin \theta \cos \theta-2 y^{\prime}\left(x_{0}-x^{\prime}\right)-2 \frac{\sin ^{3} \theta}{\cos \theta}\left(x^{\prime}-x_{0}\right)^{2}\right| \\
= & \left|-\left(t^{\prime}+2 x_{0} y^{\prime}\right)-2\left(x_{0}-x^{\prime}\right)^{2} \tan \theta-2 y^{\prime}\left(x_{0}-x^{\prime}\right)\right| .
\end{aligned}
$$

Hence, from (4.6) it follows that

$$
\begin{aligned}
\left|\tau-\tau^{\prime}\right| & \leq\left|t^{\prime}+2 x_{0} y\right|+2|\tan \theta|\left|x_{0}-x^{\prime}\right|^{2}+2\left|y^{\prime}\right|\left|x_{0}-x^{\prime}\right| \\
& \leq(1+2|\tan \theta|+2) r^{2} .
\end{aligned}
$$

For $\theta \in(0, \pi / 4]$ this yields $\sqrt{\left(\alpha-\alpha^{\prime}\right)^{2}+\left(\tau-\tau^{\prime}\right)^{2}} \leq 5 r^{2}$ which concludes the proof in this case. The proof for $\theta \in[3 \pi / 4, \pi)$ is very similar. We employ again the formula (4.7). For $\theta=0$ we have to consider a linear function instead of the quadratic function (4.7). The case $\theta \in(\pi / 4,3 \pi / 4)$ can be treated similarly, starting from a core curve of the second type.

Lemma 4.6. Let $\theta \in[0, \pi)$ and $R>0$. There exist constants $c_{1}>0$ and $c_{2}=$ $c_{2}(R)>0$ such that for all $0<r<1, z_{0}=\left|z_{0}\right| e^{\mathbf{i} \theta_{0}} \in \mathbb{C}$ with $\left|z_{0}\right| \leq R$ and $t_{0} \in \mathbb{R}$, the set

$$
p_{\mathbb{W}_{\theta}}\left(B_{\mathbb{H}}\left(\left(z_{0}, t_{0}\right), r\right)\right)
$$

can be covered by $M$ balls $B_{\mathbb{W}_{\theta}}\left(p_{j}, c_{1} r^{2}\right):=B_{\mathbb{H}}\left(p_{j}, c_{1} r^{2}\right) \cap \mathbb{W}_{\theta}, j \in\{1, \ldots, M\}$, with $M \leq c_{2} / r^{3}$.

Proof. Since the restriction of the Heisenberg metric to the vertical plane $\mathbb{W}_{\theta}$ is comparable to the parabolic heat metric on $\mathbb{R}^{2}$, there exists a constant $c_{1}>0$ such that

$$
R\left(p, r^{2}\right):=\left\{(\alpha, \tau) \in \mathbb{R}^{2}:\left|\alpha-\alpha^{\prime}\right| \leq r^{2},\left|\tau-\tau^{\prime}\right| \leq r^{4}\right\} \subseteq B_{\mathbb{W}_{\theta}}\left(p, c_{1} r^{2}\right)
$$

for all $p=\left(\alpha^{\prime}, \tau^{\prime}\right) \in \mathbb{W}_{\theta}$ and $r \geq 0$. It is therefore enough to construct a cover by rectangles $R\left(p_{j}, r^{2}\right), j \in\{1, \ldots, M\}$. 
Moreover, it suffices to prove the result for balls centered on the $x$-axis, i.e., for balls $B_{\mathbb{H}}\left(\left(z_{0}, t_{0}\right), r\right)$ with $z_{0}=x_{0} \in \mathbb{R}$ and $t_{0}=0$. Indeed, an arbitrary ball $B_{\mathbb{H}}\left(\left(z_{0}, t_{0}\right), r\right)$ can be obtained from $B_{\mathbb{H}}\left(\left(\left|z_{0}\right|, 0\right), r\right)$ by a (Euclidean) vertical translation to height $t_{0}$ and a rotation about the $t$-axis with rotation angle $\theta_{0}$. Then, as a subset of $\mathbb{R}^{2}$, the image $p_{\mathbb{W}_{\theta}}\left(B_{\mathbb{H}}\left(\left(z_{0}, t_{0}\right), r\right)\right)$ coincides with a vertical translation of $p_{\mathbb{W}_{\theta-\theta_{0}}}\left(B_{\mathbb{H}}\left(\left(\left|z_{0}\right|, 0\right), r\right)\right)$.

Let us consider a ball with radius $r<1$, centered at a point $p_{0}=\left(x_{0}, 0\right)$ with $x_{0} \in \mathbb{R},\left|x_{0}\right|<R$. The goal is to efficiently cover the set

$$
S_{\theta}\left(x_{0}, r\right):=\left\{(\alpha, \tau) \in \mathbb{R}^{2}: \alpha \in I_{\theta}^{x_{0}, r}, f_{\theta}^{x_{0}, r}(\alpha)-5 r^{2} \leq \tau \leq f_{\theta}^{x_{0}, r}(\alpha)+5 r^{2}\right\} \subseteq \mathbb{W}_{\theta}
$$

which, by Lemma 4.5 , contains the image of $B_{\mathbb{H}}\left(p_{0}, r\right)$ under $p_{\mathbb{W}_{\theta}}$, by rectangles $R\left(p_{j}, r^{2}\right), j \in\{1, \ldots, M\}$.

Let us assume that $f_{\theta}^{x_{0}, r}$ is defined on the entire real line. For given $\theta$ and $x_{0}$, we fix a particular point

$$
\alpha_{0}:=\left\{\begin{array}{cl}
-\frac{x_{0}}{\sin \theta}, & \text { if } \theta \in\left(0, \frac{\pi}{4}\right] \cup\left[\frac{3 \pi}{4}, \pi\right), \\
0, & \text { otherwise. }
\end{array}\right.
$$

In the case where $f_{\theta}^{x_{0}, r}$ is a quadratic function, it has an extremal point at $\alpha_{0}$. This is shown in the proof of Lemma 4.5 for the case $\theta \in(0, \pi / 4] \cup[3 \pi / 4, \pi)$. We write

$$
\left(I_{\theta}^{x_{0}, r}\right)_{k}:=\left[\alpha_{0}+k r^{2}, \alpha_{0}+(k+1) r^{2}\right) .
$$

It can be checked that the interval $I_{\theta}^{x_{0}, r}$ has length at most $4 r$ (this is done explicitly in the proof of Lemma 4.5 for the case $\theta \in(0, \pi / 4])$, whereas each interval of the form $\left(I_{\theta}^{x_{0}, r}\right)_{k}$ has length $r^{2}$. It follows that $I_{\theta}^{x_{0}, r}$ has nonempty intersection with at most

$$
N \leq \frac{6}{r}
$$

of the disjoint intervals $\left(I_{\theta}^{x_{0}, r}\right)_{k}$. Let $k$ be such that $I_{\theta}^{x_{0}, r} \cap\left(I_{\theta}^{x_{0}, r}\right)_{k} \neq \emptyset$. Consider now the portion of $S_{\theta}\left(x_{0}, r\right)$ which lies above the interval $\left(I_{\theta}^{x_{0}, r}\right)_{k}$, more precisely,

$$
\left\{(\alpha, \tau) \in S_{\theta}\left(x_{0}, r\right): \alpha \in\left(I_{\theta}^{x_{0}, r}\right)_{k}\right\} .
$$

In order to see how many rectangles $R\left(p_{j}, r^{2}\right)$ we need to cover this set, we have to estimate its vertical height. Direct computations for the several possible cases show that there exists a constant $c_{0}=c_{0}(R)$ such that for each $k \in \mathbb{Z}$ with $I_{\theta}^{x_{0}, r} \cap\left(I_{\theta}^{x_{0}, r}\right)_{k} \neq \emptyset$ there is an interval $\left(J_{\theta}^{x_{0}, r}\right)_{k}$ of length $c_{0} r^{2}$ with

$$
\left\{(\alpha, \tau) \in S_{\theta}\left(x_{0}, r\right): \alpha \in\left(I_{\theta}^{x_{0}, r}\right)_{k}\right\} \subseteq\left(I_{\theta}^{x_{0}, r}\right)_{k} \times\left(J_{\theta}^{x_{0}, r}\right)_{k} .
$$

Hence, because of (4.5) and (4.10), there exists an integer $N^{\prime} \in \mathbb{N}$ and points

$$
p_{k, l}=\left(\alpha_{k, l}, \tau_{k, l}\right), \quad l \in\left\{1, \ldots, N^{\prime}\right\}
$$

in the vertical plane $\mathbb{W}_{\theta}$ with

$$
N^{\prime} \leq \frac{c_{0}+1}{r^{2}}
$$


such that

$$
p_{\mathbb{W}_{\theta}}\left(B_{\mathbb{H}}\left(\left(x_{0}, 0\right), r\right)\right) \cap\left(\left(I_{\theta}^{x_{0}, r}\right)_{k} \times \mathbb{R}\right) \subseteq \bigcup_{l=1}^{N^{\prime}} R\left(p_{k, l}, r^{2}\right) .
$$

From (4.9) and (4.11) it follows that the image $p_{\mathbb{W}_{\theta}}\left(B_{\mathbb{H}}\left(\left(x_{0}, 0\right), r\right)\right)$ can be covered by

$$
M:=N \cdot N^{\prime} \leq \frac{6\left(c_{0}+1\right)}{r^{3}}
$$

rectangles $R\left(p_{j}, r^{2}\right)=R\left(p_{k, l}, r^{2}\right)$. Since each of these rectangles is contained in a ball $B_{\mathbb{W}}\left(p_{j}, c_{1} r^{2}\right)$, this concludes the proof of Lemma 4.6.

Proof of Proposition 4.3. We may without loss of generality assume that the set $A$ is bounded. We denote its Hausdorff dimension by $\operatorname{dim}_{\mathbb{H}} A=s \in[1,3]$. Then we have $\mathcal{H}_{\mathbb{H}}^{s+\varepsilon}(A)=0$ for all $\varepsilon>0$ and thus, for each $\varepsilon>0$,

$$
\left(\mathcal{H}_{\mathbb{H}}\right)_{\delta}^{s+\varepsilon}(A)=0 \text { for all } \delta>0 .
$$

Hence, for all $\varepsilon>0$ and $0<\delta<1$, there exists a countable collection of balls

$$
B_{\mathbb{H}}\left(p_{i}, r_{i}\right), \quad i \in \mathbb{N}, \quad r_{i} \leq \delta,
$$

with

$$
A \subseteq \bigcup_{i \in \mathbb{N}} B_{\mathbb{H}}\left(p_{i}, r_{i}\right), \quad \sum_{i=1}^{\infty} r_{i}^{s+2 \varepsilon}<\delta .
$$

We write $p_{i}=\left(z_{i}, t_{i}\right)=\left(\left|z_{i}\right| e^{\mathbf{i} \theta_{0, i}}, t_{i}\right)$. Since the set $A$ is bounded, we may assume that there exists $R>0$ such that $\left|z_{i}\right| \leq R$ for all $i \in \mathbb{N}$.

Fix now $\theta \in[0, \pi)$. It follows from Lemma 4.6 that there exist $c_{1}, c_{2}>0$ (independent of $p_{i}$ and $r_{i}$ ), constants $M_{i}$ with $M_{i} \leq c_{2} / r_{i}^{3}$, and points $p_{i, j}, i \in \mathbb{N}$ and $j \in\left\{1, \ldots, M_{i}\right\}$ such that

$$
p_{\mathbb{W}_{\theta}}(A) \subseteq \bigcup_{i \in \mathbb{N}} \bigcup_{j=1}^{M_{i}} B_{\mathbb{W}_{\theta}}\left(p_{i, j}, c_{1} r_{i}^{2}\right) .
$$

For $\sigma \geq 0$, notice that

$$
\begin{aligned}
\sum_{i, j} \operatorname{diam}\left(B_{\mathbb{W}_{\theta}}\left(p_{i, j}, c_{1} r_{i}^{2}\right)\right)^{\sigma+\varepsilon} & =\sum_{i \in \mathbb{N}} \sum_{j=1}^{M_{i}}\left(2 c_{1} r_{i}^{2}\right)^{\sigma+\varepsilon}=\sum_{i \in \mathbb{N}} M_{i}\left(2 c_{1}\right)^{\sigma+\varepsilon} r_{i}^{2 \sigma+2 \varepsilon} \\
& \leq\left(2 c_{1}\right)^{\sigma+\varepsilon} c_{2} \sum_{i \in \mathbb{N}} r_{i}^{(2 \sigma-3)+2 \varepsilon} .
\end{aligned}
$$

Now if $\sigma$ is chosen such that $2 \sigma-3=s$, i.e.,

$$
\sigma=\frac{1}{2}(s+3)=\frac{1}{2}\left(\operatorname{dim}_{\mathbb{H}} A+3\right),
$$

it follows

$$
\sum_{i, j} \operatorname{diam}\left(B_{\mathbb{W}_{\theta}}\left(p_{i, j}, c_{1} r_{i}^{2}\right)\right)^{\sigma+\varepsilon}<\left(2 c_{1}\right)^{\sigma+\varepsilon} c_{2} \delta
$$


From (4.13) and (4.14), we conclude that

$$
\left(\mathcal{H}_{\mathbb{H}}\right)_{2 c_{1} \delta^{2}}^{\sigma+\varepsilon}\left(p_{\mathbb{W}_{\theta}}(A)\right) \leq\left(2 c_{1}\right)^{\sigma+\varepsilon} c_{2} \delta .
$$

Letting $\delta$ tend to zero yields

$$
\mathcal{H}_{\mathbb{H}}^{\sigma+\varepsilon}\left(p_{\mathbb{W}_{\theta}}(A)\right)=0
$$

and thus,

$$
\operatorname{dim}_{\mathbb{H}} p_{\mathbb{W}_{\theta}}(A) \leq \sigma=\frac{1}{2}\left(\operatorname{dim}_{\mathbb{H}} A+3\right),
$$

as desired. This concludes the proof of Proposition 4.3.

Next, we discuss universal lower dimension bounds for vertical projections. We will prove two propositions. Proposition 4.7 is the vertical analog of Lemma 3.1. Observe that the failure of the vertical projection to be Lipschitz resurfaces in the proof of this result; see (4.15). Proposition 4.9 uses a slicing theorem for dimensions of intersections of sets with planes in Euclidean space together with the dimension comparison principle. Taken together, these two propositions establish the universal lower bounds in Theorem 1.3.

Proposition 4.7. Let $A \subset \mathbb{H}$ be Borel with $\operatorname{dim}_{\mathbb{H}} A \geq 1$. Then, for every $\theta$,

$$
\operatorname{dim}_{\mathbb{H}} p_{\mathbb{W}_{\theta}}(A) \geq \frac{1}{2}\left(\operatorname{dim}_{\mathbb{H}} A-1\right) .
$$

In the proof, we use the following elementary estimate whose proof we omit. Compare Lemma 4.4 in [10].

Lemma 4.8. There exists an absolute constant $C>0$ so that

$$
\left\|a^{-1} * b * a\right\|_{\mathbb{H}}^{4} \leq\|b\|_{\mathbb{H}}^{4}+C\|b\|_{\mathbb{H}}^{2}
$$

whenever $a$ and $b$ are points in $\mathbb{H}$ with $\|a\|_{\mathbb{H}} \leq 1$ and $\|b\|_{\mathbb{H}} \leq 1$.

Proof of Proposition 4.7. We may assume without loss of generality that $A$ is bounded. In fact, let us assume that $|z| \leq 1$ for all points $p=(z, t) \in A$.

Fix $\theta \in[0, \pi)$, let $s>\operatorname{dim}_{\mathbb{H}} p_{\mathbb{W}_{\theta}}(A)$, let $\varepsilon>0$, and cover the set $p_{\mathbb{W}_{\theta}}(A)$ with a family of Heisenberg balls $\left\{B_{\mathbb{H}}\left(\left(z_{i}, t_{i}\right), r_{i}\right)\right\}_{i}$ so that $\sum_{i} r_{i}^{s}<\varepsilon$.

Claim: We can choose $C_{0}>0$ and a number $N_{i} \leq C_{0} r_{i}^{-1 / 2}$ of values $\left(z_{i j}, t_{i j}\right)$ contained in the fiber $p_{\mathbb{W}_{\theta}}^{-1}\left(z_{i}, t_{i}\right)$ so that the family $\left\{B_{\mathbb{H}}\left(\left(z_{i j}, t_{i j}\right), C_{0} \sqrt{r_{i}}\right)\right\}_{j}$ covers the set $p_{\mathbb{W}_{\theta}}^{-1}\left(B_{\mathbb{H}}\left(\left(z_{i}, t_{i}\right), r_{i}\right)\right)$.

To prove the claim, it suffices to show that

$$
p_{\mathbb{W}_{\theta}}^{-1}\left(B_{\mathbb{H}}(q, r) \cap \mathbb{W}_{\theta}\right) \cap B_{\mathbb{H}}((0,0), 1) \subset N_{\mathbb{H}}\left(q * \mathbb{V}_{\theta}, C \sqrt{r}\right)
$$

for some constant $C>0$, whenever $q \in \mathbb{W}_{\theta}$ and $0<r \leq 1$. Here $N_{\mathbb{H}}(S, \delta)$ denotes the $\delta$-neighborhood of a set $S \subset \mathbb{H}$ in the metric $d_{\mathbb{H}}$, that is,

$$
N_{\mathbb{H}}(S, \delta)=\bigcup_{s \in S} B_{\mathbb{H}}(s, \delta) .
$$


The inclusion in (4.15) is a consequence of the following statement:

For all $q^{\prime} \in \mathbb{W}_{\theta}$ so that $d_{\mathbb{H}}\left(q, q^{\prime}\right) \leq r$ and for all $p^{\prime} \in \mathbb{V}_{\theta}$ so that $\left\|p^{\prime}\right\|_{\mathbb{H}} \leq 1$, there exists $p \in \mathbb{V}_{\theta}$ so that $d_{\mathbb{H}}\left(q^{\prime} * p^{\prime}, q * p\right) \leq C \sqrt{r}$.

To establish this statement, choose $p=p^{\prime}$. Then

$$
d_{\mathbb{H}}\left(q^{\prime} * p^{\prime}, q * p\right)^{4}=\left\|p^{-1} *\left(q^{-1} * q^{\prime}\right) * p\right\|_{\mathbb{H}}^{4} \leq d_{\mathbb{H}}\left(q, q^{\prime}\right)^{4}+C d_{\mathbb{H}}\left(q, q^{\prime}\right)^{2}
$$

by Lemma 4.8. Since $d_{\mathbb{H}}\left(q, q^{\prime}\right) \leq r$ by assumption and $r \leq 1$, we conclude that

$$
d_{\mathbb{H}}\left(q^{\prime} * p^{\prime}, q * p\right)^{4} \leq C r^{2}
$$

which finishes the proof of the claim.

With the claim in hand, the rest of the proof of Proposition 4.7 proceeds exactly as for its horizontal counterpart. The family $\left\{B_{\mathbb{H}}\left(\left(z_{i j}, t_{i j}\right), C_{0} \sqrt{r_{i}}\right)\right\}_{i, j}$ covers the set $A$ and we compute

$$
\sum_{i, j} \operatorname{rad}\left(B_{\mathbb{H}}\left(\left(z_{i j}, t_{i j}\right), C_{0} \sqrt{r_{i}}\right)\right)^{2 s+1}=\sum_{i} N_{i}\left(C_{0} \sqrt{r_{i}}\right)^{2 s+1} \leq C_{0}^{2 s+2} \sum_{i} r_{i}^{s} \leq C_{0}^{2 s+2} \varepsilon .
$$

Letting $\varepsilon \rightarrow 0$ gives $\mathcal{H}_{\mathbb{H}}^{2 s+1}(A)=0$, so $\operatorname{dim}_{\mathbb{H}} A \leq 2 s+1$. Letting $s$ tend to $\operatorname{dim}_{\mathbb{H}} p_{\mathbb{W}_{\theta}}(A)$ completes the proof.

Proposition 4.9. Let $A \subset \mathbb{H}$ be Borel with $\operatorname{dim}_{\mathbb{H}} A \geq 3$. Then

$$
\operatorname{dim}_{\mathbb{H}} p_{\mathbb{W}_{\theta}}(A) \geq 2 \operatorname{dim}_{\mathbb{H}} A-5 \quad \text { for every } \theta .
$$

Proof. It suffices to assume that $\operatorname{dim}_{\mathbb{H}} A>3$. By the dimension comparison principle,

$$
\operatorname{dim}_{E} A \geq \operatorname{dim}_{\mathbb{H}} A-1>2 .
$$

Let $0<\varepsilon<\operatorname{dim}_{\mathbb{H}} A-3$. According to the classical Euclidean intersection theorem (see Theorem 10.10 in [18]), there exists a plane $\Pi$ in $\mathbb{R}^{3}$ for which

$$
\operatorname{dim}_{E}(A \cap \Pi) \geq \operatorname{dim}_{E} A-1-\varepsilon \geq \operatorname{dim}_{\mathbb{H}} A-2-\varepsilon>1 .
$$

Furthermore, we may assume that $\Pi$ is not a vertical plane, i.e., $\Pi$ is a $t$-graph: the graph of a function $u: \mathbb{C} \rightarrow \mathbb{R}$. Let us write

$$
\Pi=\{(z, t): t=u(z):=2 \operatorname{Re}(\bar{a} z)+b\}
$$

for some $a \in \mathbb{C}$ and $b \in \mathbb{R}$. Consider the map $F: \mathbb{R}^{2} \rightarrow \mathbb{R}^{2}$ given as the composition of the graph map id $\otimes u$, the vertical projection $p_{\mathbb{W}_{\theta}}$, and the coordinate chart $\varphi_{\mathbb{W}_{\theta}}$ (see (1.3)). Written in complex notation,

$$
F(z)=\left(\operatorname{Im}\left(e^{-\mathbf{i} \theta} z\right), 2 \operatorname{Re}(\bar{a} z)+b-\operatorname{Im}\left(e^{-2 \mathbf{i} \theta} z^{2}\right)\right) .
$$

The Jacobian determinant of $F$ is given by

$$
\operatorname{det} D F(z)=2 \operatorname{Im}\left(e^{-\mathbf{i} \theta}(z-\mathbf{i} a)\right)
$$

and the restriction of $p_{\mathbb{W}_{\theta}}$ to $\{(z, u(z)) \in \Pi: \operatorname{det} D F(z) \neq 0\}$ is locally bi-Lipschitz. 
Observe that

$$
\gamma=\{(z, u(z)) \in \Pi: \operatorname{det} D F(z)=0\}
$$

is a line. Since $\operatorname{dim}_{E}(A \cap \Pi)>1, \operatorname{dim}_{E}(A \cap(\Pi \backslash \gamma))=\operatorname{dim}_{E}(A \cap \Pi)$, and it follows that

$$
\begin{aligned}
\operatorname{dim}_{E} p_{\mathbb{W}_{\theta}}(A) & \geq \operatorname{dim}_{E} p_{\mathbb{W}_{\theta}}(A \cap(\Pi \backslash \gamma)) \\
& =\operatorname{dim}_{E}(A \cap(\Pi \backslash \gamma))=\operatorname{dim}_{E}(A \cap \Pi) \geq \operatorname{dim}_{\mathbb{H}} A-2-\varepsilon
\end{aligned}
$$

To complete the proof, we use the dimension comparison principle again to switch back from the Euclidean dimension of the projected set to its Heisenberg dimension. Since $p_{\mathbb{W}_{\theta}}(A)$ is contained in $\mathbb{W}_{\theta}$, we can use the improved lower dimension comparison bound from Theorem 2.8. Using (4.16) we obtain

$$
\operatorname{dim}_{\mathbb{H}} p_{\mathbb{W}_{\theta}}(A) \geq \beta_{-}^{\mathbb{W}}\left(\operatorname{dim}_{E} p_{\mathbb{W}_{\theta}}(A)\right) \geq \beta_{-}^{\mathbb{W}}\left(\operatorname{dim}_{\mathbb{H}} A-2-\varepsilon\right),
$$

and thus, letting $\varepsilon$ tend to zero,

$$
\operatorname{dim}_{\mathbb{H}} p_{\mathbb{W}_{\theta}}(A) \geq 2 \operatorname{dim}_{\mathbb{H}} A-5,
$$

as asserted in the statement. The proof is complete.

The result of Theorem 1.3 follows by combining Propositions 4.2, 4.7 and 4.9.

We now turn to the proof of the sharpness statement of Theorem 1.3.

Proposition 4.10. In each of the following statements, the set $A$ is a compact subset of $\mathbb{H}$ :

(a) For all $0 \leq \beta \leq 1$ there exists $A$ so that

$$
\operatorname{dim}_{\mathbb{H}} A=\beta \quad \text { and } \quad \operatorname{dim}_{\mathbb{H}} p_{\mathbb{W}_{0}}(A)=0 .
$$

(b) For all $1 \leq \beta \leq 3$ there exists $A$ so that

$$
\operatorname{dim}_{\mathbb{H}} A=\beta \quad \text { and } \quad \operatorname{dim}_{\mathbb{H}} p_{\mathbb{W}_{0}}(A)=(\beta-1) / 2 .
$$

(c) For all $3 \leq \beta \leq 4$ there exists $A$ so that

$$
\operatorname{dim}_{\mathbb{H}} A=\beta \quad \text { and } \quad \operatorname{dim}_{\mathbb{H}} p_{\mathbb{W}_{0}}(A)=2 \beta-5 .
$$

(d) For all $0 \leq \beta \leq 1$ there exists $A$ so that

$$
\operatorname{dim}_{\mathbb{H}} A=\beta \quad \text { and } \quad \operatorname{dim}_{\mathbb{H}} p_{\mathbb{W}_{0}}(A)=2 \beta .
$$

(e) For all $1 \leq \beta \leq 3$ there exists $A$ so that

$$
\operatorname{dim}_{\mathbb{H}} A=\beta \quad \text { and } \quad \operatorname{dim}_{\mathbb{H}} p_{\mathbb{W}_{0}}(A)=\frac{1}{2}(\beta+3) .
$$

(f) For all $3 \leq \beta \leq 4$ there exists $A$ so that

$$
\operatorname{dim}_{\mathbb{H}} A=\beta \quad \text { and } \quad \operatorname{dim}_{\mathbb{H}} p_{\mathbb{W}_{0}}(A)=3 .
$$


Proof of Proposition 4.10. For a proof of statements (d), (e) and (f), i.e., for the sharpness of the upper dimension bounds, see the proof of Proposition 5.3, where sets are constructed for which the corresponding dimension values hold for all directions $\theta$, and not merely for $\theta=0$. In the following, we discuss the sharpness of the lower dimension bound, i.e., the cases (a), (b) and (c).

Assume that $0 \leq \beta \leq 1$. Let $A \subset \mathbb{V}_{0}$ be a compact $\beta$-set. Then the set $p_{\mathbb{W}_{0}}(A)=\{(0,0)\}$ is zero-dimensional. This gives an example of a set $A$ satisfy$\operatorname{ing}(\mathrm{a})$.

Examples for (b) and (c) are based on the following special case $(\beta=3)$, which we describe first. Let $B_{0}=\{(\mathbf{i} y, 0): y \in \mathbb{R}\}$ be the $y$ axis and let

$$
A_{0}=p_{\mathbb{W}_{0}}^{-1}\left(B_{0}\right)=\{(x+\mathbf{i} y, 2 x y): x, y \in \mathbb{R}\} .
$$

Then $\operatorname{dim}_{\mathbb{H}} B_{0}=1$, while $\operatorname{dim}_{\mathbb{H}} A_{0}=3$.

Next, assume that $1<\beta<3$; we construct a set $A$ satisfying (b). The desired set is constructed as a subset of the set $A_{0}$ defined in (4.17). Let $S \subset \mathbb{R}$ be a compact Ahlfors regular set of dimension $(\beta-1) / 2$ and consider the set

$$
A=p_{\mathbb{W}_{0}}^{-1}(\{(\mathbf{i} y, 0): y \in S\}) .
$$

Clearly $\operatorname{dim}_{\mathbb{H}} p_{\mathbb{W}_{0}}(A)=\operatorname{dim}_{E} p_{\mathbb{W}_{0}}(A)=(\beta-1) / 2$. By Theorem 1.3,

$$
\frac{1}{2}\left(\operatorname{dim}_{\mathbb{H}} A-1\right) \leq \operatorname{dim}_{\mathbb{H}} p_{\mathbb{W}_{0}}(A),
$$

so it suffices to verify that $\operatorname{dim}_{\mathbb{H}} A \geq \beta$. Again we will appeal to Theorem 2.4 ; the details are similar to those in the proof of Proposition $3.2(\mathrm{~d})$.

Define a set function $\mu$ on $A$ by

$$
\mu(E)=\int_{S} \mathcal{H}_{\mathbb{H}}^{1}\left(E \cap L_{y}\right) d \mathcal{H}_{E}^{(\beta-1) / 2}(y), \quad \text { for Borel sets } E \subseteq A,
$$

where $L_{y}:=p_{\mathbb{W}_{0}}^{-1}(\mathbf{i} y, 0)$. Let $B_{\mathbb{H}}(p, r)$ be a ball in $\left(\mathbb{H}, d_{\mathbb{H}}\right)$ centered at $p \in A$ with radius $r$. Write $p=\left(x_{0}+\mathbf{i} y_{0}, 2 x_{0} y_{0}\right)$ for some $y_{0} \in S$ and $x_{0} \in \mathbb{R}$.

Lemma 4.11. If $B_{\mathbb{H}}(p, r) \cap L_{y} \neq \emptyset$, then $\left|y-y_{0}\right| \leq r^{2}$ and

$$
\mathcal{H}_{\mathbb{H}}^{1}\left(B_{\mathbb{H}}(p, r) \cap L_{y}\right) \leq C r,
$$

for a constant $C$ independent of $p, r$ and $y$.

Assuming the lemma we complete the proof in this case:

$$
\begin{aligned}
\mu\left(B_{\mathbb{H}}(p, r)\right) & \leq C r \cdot \mathcal{H}_{E}^{(\beta-1) / 2}\left(\left\{y: B_{\mathbb{H}}(p, r) \cap L_{y} \neq \emptyset\right\}\right) \\
& \leq C r \cdot \mathcal{H}_{E}^{(\beta-1) / 2}\left(\left[y_{0}-r^{2}, y_{0}+r^{2}\right] \cap A\right) \leq C^{\prime} r^{\beta}
\end{aligned}
$$

for $C^{\prime}>0$ independent of $p$ and $r$. Hence $\mu$ satisfies the upper mass bound $(2.2)$ on $A$ with exponent $\beta$. By Theorem 2.4, $\operatorname{dim}_{\mathbb{H}} A \geq \beta$. 
It remains to prove the lemma. Suppose that $B_{\mathbb{H}}(p, r) \cap L_{y} \neq \emptyset$. Then $B_{\mathbb{H}}(p, r) \subset B_{\mathbb{H}}(q, 2 r)$ for some $q \in B_{\mathbb{H}}(p, r) \cap L_{y}$ and

$$
\mathcal{H}_{\mathbb{H}}^{1}\left(B_{\mathbb{H}}(p, r) \cap L_{y}\right) \leq \mathcal{H}_{\mathbb{H}}^{1}\left(B_{\mathbb{H}}(q, 2 r) \cap L_{y}\right) \leq C r
$$

since $L_{y}$ is a horizontal line. Furthermore, if $q=(x+\mathbf{i} y, 2 x y)$ then

$$
p^{-1} * q=\left(\left(x-x_{0}\right)+\mathbf{i}\left(y-y_{0}\right), 2\left(x+x_{0}\right)\left(y-y_{0}\right)\right) .
$$

Since $d_{\mathbb{H}}(p, q) \leq r$ we conclude

$$
\left|x-x_{0}\right| \leq r \quad \text { and } \quad\left|2\left(x+x_{0}\right)\left(y-y_{0}\right)\right| \leq r^{2} .
$$

We may restrict to the subset of $A$ consisting of points $(x+\mathbf{i} y, t)$ for which $|x| \geq 1$ and consider only radii $r<1$. Then

$$
\left|x+x_{0}\right| \geq 2|x|-\left|x-x_{0}\right| \geq 2-r>1
$$

and so

$$
\left|y-y_{0}\right| \leq C r^{2}
$$

This completes the proof of Lemma 4.11 and ends the construction when $\beta \in[1,3]$.

Finally, assume that $3<\beta \leq 4$. The desired set in this case is constructed as a union of a collection of vertical translates of the set $A_{0}$ defined in (4.17). Let $S \subset \mathbb{R}$ be a compact Ahlfors regular set of dimension $\beta-3$ and let

$$
A=\bigcup_{s \in S} \tau_{(0, s)}\left(A_{0}\right)
$$

where $\tau_{q}: \mathbb{H} \rightarrow \mathbb{H}, \tau_{q}(p)=q * p$, denotes left translation by $q \in \mathbb{H}$. Then $A \subset \mathbb{H}$ is compact and $p_{\mathbb{W}_{0}}(A) \supset\{(\mathbf{i} y, s): y \in[0,1], s \in S\}$, whence

$$
\operatorname{dim}_{\mathbb{H}} p_{\mathbb{W}_{0}}(A) \geq 1+2(\beta-3)=2 \beta-5 .
$$

By Theorem 1.3, $2 \operatorname{dim}_{\mathbb{H}} A-5 \leq \operatorname{dim}_{\mathbb{H}} p_{\mathbb{W}_{0}}(A)$, so it suffices to verify that $\operatorname{dim}_{\mathbb{H}} A \geq \beta$. Define a set function $\mu$ on $A$ by setting

$$
\mu(E)=\int_{S} \mathcal{H}_{\mathbb{H}}^{3}\left(E \cap \Sigma_{s}\right) d \mathcal{H}_{E}^{\beta-3}(s), \quad \text { for Borel sets } E \subseteq A
$$

where $\Sigma_{s}=\tau_{(0, s)}\left(A_{0}\right)$. Let $B_{\mathbb{H}}(p, r)$ be a ball in $\left(\mathbb{H}, d_{\mathbb{H}}\right)$ centered at $p \in A$ with radius $r$. Write $p=\left(x_{0}+\mathbf{i} y_{0}, 2 x_{0} y_{0}+s_{0}\right)$ for some $s_{0} \in S$ and $x_{0}, y_{0} \in \mathbb{R}$. Then $p \in \Sigma_{s}$.

Lemma 4.12. If $B_{\mathbb{H}}(p, r) \cap \Sigma_{s} \neq \emptyset$, then $\left|s-s_{0}\right| \leq C r$ and

$$
\mathcal{H}_{\mathbb{H}}^{3}\left(B_{\mathbb{H}}(p, r) \cap \Sigma_{s}\right) \lesssim C r^{3} .
$$


Assuming the lemma we complete the proof in this case:

$$
\begin{aligned}
\mu\left(B_{\mathbb{H}}(p, r)\right) & \leq C r^{3} \cdot \mathcal{H}_{E}^{\beta-3}\left(\left\{s: B_{\mathbb{H}}(p, r) \cap \Sigma_{s} \neq \emptyset\right\}\right) \\
& \leq C r^{3} \cdot \mathcal{H}_{E}^{\beta-3}\left(\left[s_{0}-r, s_{0}+r\right] \cap A\right) \leq C r^{\beta} .
\end{aligned}
$$

Hence $\mu$ satisfies the upper mass bound (2.2) on $A$ with exponent $\beta$. By Theorem 2.4, $\operatorname{dim}_{\mathbb{H}} A \geq \beta$.

It remains to prove the lemma. Suppose that $B_{\mathbb{H}}(p, r) \cap \Sigma_{s} \neq \emptyset$. Then $B_{\mathbb{H}}(p, r) \subset B_{\mathbb{H}}(q, 2 r)$ for some $q \in B_{\mathbb{H}}(p, r) \cap \Sigma_{s}$ and

$$
\mathcal{H}_{\mathbb{H}}^{3}\left(B_{\mathbb{H}}(p, r) \cap \Sigma_{s}\right) \leq \mathcal{H}_{\mathbb{H}}^{3}\left(B_{\mathbb{H}}(q, 2 r) \cap \Sigma_{s}\right) \leq C r^{3}
$$

since $\Sigma_{s}$ is a smooth submanifold. Furthermore, if $q=(x+\mathbf{i} y, 2 x y+s)$ then

$$
p^{-1} * q=\left(\left(x-x_{0}\right)+\mathbf{i}\left(y-y_{0}\right),\left(s-s_{0}\right)+2\left(x+x_{0}\right)\left(y-y_{0}\right)\right) .
$$

Since $d_{\mathbb{H}}(p, q) \leq r$ we conclude

$$
\left|y-y_{0}\right| \leq r \quad \text { and } \quad\left|\left(s-s_{0}\right)+2\left(x+x_{0}\right)\left(y-y_{0}\right)\right| \leq r^{2}
$$

and so

$$
\left|s-s_{0}\right| \leq r^{2}+C\left|y-y_{0}\right| \leq C r .
$$

This completes the proof of Lemma 4.12, and ends the construction in the case $\beta \in[3,4]$.

\section{Almost sure bounds for vertical projections}

The goal of this section is to prove an almost sure lower bound for vertical projections (Theorem 1.4) and to verify that the given universal upper bound is sharp even as an almost sure statement.

The arguments concerning the lower bound go along the lines of the proof of the corresponding Euclidean result. However, it is considerably more difficult to establish the integrability of certain functions given in terms of the Heisenberg distance between projected points and the proof works only for a restricted range of dimensions, namely, $\operatorname{dim}_{\mathbb{H}} A \leq 1$.

Here is the main proposition of this section.

Proposition 5.1. Let $A \subset \mathbb{H}$ be a Borel set with $\operatorname{dim}_{\mathbb{H}} A \leq 1$. Then, for a.e. $\theta$,

$$
\operatorname{dim}_{\mathbb{H}} p_{\mathbb{W}_{\theta}}(A) \geq \operatorname{dim}_{\mathbb{H}} A .
$$

Corollary 5.2. Let $A \subset \mathbb{H}$ be a Borel set. Then, for a.e. $\theta$,

$$
\operatorname{dim}_{\mathbb{H}} p_{\mathbb{W}_{\theta}}(A) \geq \min \left\{\operatorname{dim}_{\mathbb{H}} A, 1\right\} .
$$

To prove the corollary, let $A$ be a Borel subset of $\mathbb{H}$ with $\operatorname{dim}_{\mathbb{H}} A>1$ and choose a subset $B \subset A$ with $\operatorname{dim}_{\mathbb{H}} B=1$. For almost every parameter $\theta$, we have $\operatorname{dim}_{\mathbb{H}} p_{\mathbb{W}_{\theta}}(A) \geq \operatorname{dim}_{\mathbb{H}} p_{\mathbb{W}_{\theta}}(B) \geq \operatorname{dim}_{\mathbb{H}} B=1$. 
Proof of Proposition 5.1. Fix $0<\sigma<\operatorname{dim}_{\mathbb{H}} A$. By Theorem 2.6, there exists $\mu \in \mathcal{M}(A)$ with

$$
I_{\sigma}(\mu)=\int_{A} \int_{A} d_{\mathbb{H}}(p, q)^{-\sigma} \mathrm{d} \mu(p) \mathrm{d} \mu(q)<\infty .
$$

Using this measure, we will define a family of measures $\left\{\mu_{\theta}\right\}_{\theta \in[0, \pi)}$ so that $\mu_{\theta} \in$ $\mathcal{M}\left(p_{\mathbb{W}_{\theta}}(A)\right)$ and

$$
\int_{0}^{\pi} I_{\sigma}\left(\mu_{\theta}\right) \mathrm{d} \theta<\infty
$$

Once this done, the proof is finished by another appeal to Theorem 2.6 (since the integrand of (5.1) must be finite for almost every $\theta$ ) and by taking the limit as $\sigma$ increases to $\operatorname{dim}_{\mathbb{H}} A$.

It remains to construct the measures $\mu_{\theta}$ and verify (5.1). Consider the pushforward measure $\mu_{\theta}:=\left(p_{\mathbb{W}_{\theta}}\right)_{\sharp} \mu$ defined by

$$
\left(p_{\mathbb{W}_{\theta}}\right)_{\sharp} \mu(E)=\mu\left(p_{\mathbb{W}_{\theta}}^{-1}(E)\right) .
$$

It is not hard to see that $\mu_{\theta}$ is in $\mathcal{M}\left(p_{\mathbb{W}_{\theta}}(A)\right)$. By Fubini's theorem and the definition of the pushforward measure, the integral in (5.1) is equal to

$$
\int_{A} \int_{A} \int_{0}^{\pi} d_{\mathbb{H}}\left(p_{\mathbb{W}_{\theta}}(p), p_{\mathbb{W}_{\theta}}(q)\right)^{-\sigma} \mathrm{d} \theta \mathrm{d} \mu(p) \mathrm{d} \mu(q) .
$$

We claim that the quantity in (5.2) is bounded above by an absolute constant multiple of $I_{\sigma}(\mu)$, i.e.,

$$
\begin{aligned}
& \int_{A} \int_{A} \int_{0}^{\pi} d_{\mathbb{H}}\left(p_{\mathbb{W}_{\theta}}(p),\right.\left.p_{\mathbb{W}_{\theta}}(q)\right)^{-\sigma} \mathrm{d} \theta \mathrm{d} \mu(p) \mathrm{d} \mu(q) \\
& \leq C \int_{A} \int_{A} d_{\mathbb{H}}(p, q)^{-\sigma} \mathrm{d} \mu(p) \mathrm{d} \mu(q) .
\end{aligned}
$$

Unlike the Euclidean case, the distance $d_{\mathbb{H}}\left(p_{\mathbb{W}_{\theta}}(p), p_{\mathbb{W}_{\theta}}(q)\right)$ is not related to the distance $d_{\mathbb{H}}(p, q)$ in any simple way. This means that we are not able to prove (5.3) by bounding the inner integral pointwise by $d_{\mathbb{H}}(p, q)^{-\sigma}$ as in the Euclidean case. The main technical difficulties in the proof lie in the verification of (5.3).

In order to prove (5.3), we split the domain of integration $A \times A$ into two pieces, according to the two terms which appear in the formula (2.8) for the Heisenberg distance. Let

$$
A_{1}:=\left\{(p, q) \in A \times A:|z-\zeta|^{2} \geq|t-\tau+| z^{2}-\zeta^{2}\left|\sin \left(\varphi_{1}-\varphi_{2}\right)\right|\right\}
$$

and

$$
A_{2}:=\left\{(p, q) \in A \times A:|z-\zeta|^{2}<|t-\tau+| z^{2}-\zeta^{2}\left|\sin \left(\varphi_{1}-\varphi_{2}\right)\right|\right\} .
$$

First, suppose that $(p, q) \in A_{1}$. We observe the following distance estimates in this case:

$$
d_{\mathbb{H}}(p, q)^{4} \leq 2|z-\zeta|^{4} \quad \text { and } \quad d_{\mathbb{H}}\left(p_{\mathbb{W}_{\theta}}(p), p_{\mathbb{W}_{\theta}}(q)\right)^{4} \geq|z-\zeta|^{4} \sin ^{4}\left(\varphi_{1}-\theta\right) .
$$


Then

$$
\int_{0}^{\pi} d_{\mathbb{H}}\left(p_{\mathbb{W}_{\theta}}(p), p_{\mathbb{W}_{\theta}}(q)\right)^{-\sigma} \mathrm{d} \theta \leq|z-\zeta|^{-\sigma} \int_{0}^{\pi} \frac{\mathrm{d} \theta}{\left|\sin \left(\varphi_{1}-\theta\right)\right|^{\sigma}} \leq C_{1} d_{\mathbb{H}}(p, q)^{-\sigma},
$$

where $C_{1}=2^{\sigma / 4} \int_{0}^{\pi}|\sin \theta|^{-\sigma} \mathrm{d} \theta<\infty$. Note that in this case we use the assumption $\sigma<1$, and also that the constant $C_{1}$ is independent of $p$ and $q$.

Next, suppose that $(p, q) \in A_{2}$. Let us introduce the abbreviated notation

$$
a:=\left|z^{2}-\zeta^{2}\right|, \quad b:=t-\tau, \quad \text { and } \quad \varphi_{0}:=\varphi_{2}-\varphi_{1} .
$$

Observe that the condition $(p, q) \in A_{2}$ implies that either $b$ is nonzero, or that both $a$ and $\sin \varphi_{0}$ are nonzero. We also have

$$
d_{\mathbb{H}}(p, q)^{4} \leq 2\left(b+a \sin \varphi_{0}\right)^{2}
$$

and

$$
d_{\mathbb{H}}\left(p_{\mathbb{W}_{\theta}}(p), p_{\mathbb{W}_{\theta}}(q)\right)^{4} \geq\left(b-a \sin \left(\varphi_{0}+2 \varphi_{1}-2 \theta\right)\right)^{2}
$$

Hence,

$$
\begin{aligned}
\int_{0}^{\pi} d_{\mathbb{H}}\left(p_{\mathbb{W}_{\theta}}(p), p_{\mathbb{W}_{\theta}}(q)\right)^{-\sigma} \mathrm{d} \theta & \leq \int_{0}^{\pi}\left|b-a \sin \left(\varphi_{0}+2 \varphi_{1}-2 \theta\right)\right|^{-\sigma / 2} \mathrm{~d} \theta \\
& =\frac{1}{2} \int_{0}^{2 \pi}|b+a \sin \theta|^{-\sigma / 2} \mathrm{~d} \theta
\end{aligned}
$$

and

$$
d_{\mathbb{H}}(p, q)^{-\sigma} \geq 2^{-\sigma / 4}\left|b+a \sin \varphi_{0}\right|^{-\sigma / 2},
$$

so it suffices to find a constant $C_{2}$ independent of $a, b$ and $\varphi_{0}$ for which

$$
\int_{0}^{2 \pi} \frac{\mathrm{d} \theta}{|b+a \sin \theta|^{\sigma / 2}} \leq \frac{C_{2}}{\left|b+a \sin \varphi_{0}\right|^{\sigma / 2}}
$$

whenever either $b \neq 0$ or $a \sin \varphi_{0} \neq 0$.

We finish the proof by verifying (5.4) for some explicit constant $C_{2}$. If $a=0$, then (5.4) is satisfied for $C_{2}=2 \pi$, so assume $a \neq 0$. Then (5.4) is equivalent to

$$
\int_{0}^{2 \pi} \frac{\mathrm{d} \theta}{|r+\sin \theta|^{\sigma / 2}} \leq \frac{C_{2}}{\left|r+\sin \varphi_{0}\right|^{\sigma / 2}}
$$

where $r=b / a$. By a change of variables, we may assume without loss of generality that $r \geq 0$. Observe that (5.5) is implied by

$$
\int_{0}^{2 \pi} \frac{\mathrm{d} \theta}{|r+\sin \theta|^{\sigma / 2}} \leq \frac{C_{2}}{(1+r)^{\sigma / 2}}
$$

so we are reduced to verifying (5.6). 
Consider the function

$$
g(r):=(1+r)^{\sigma / 2} \int_{0}^{2 \pi} \frac{\mathrm{d} \theta}{|r+\sin \theta|^{\sigma / 2}} .
$$

A straightforward computation shows that $g$ is monotone decreasing on the interval $[1, \infty)$, with $g(1)=2^{\sigma / 2} \int_{0}^{2 \pi}(1+\sin \theta)^{-\sigma / 2} \mathrm{~d} \theta$, which is finite since $\sigma<1$. Suppose that $0 \leq r<1$ and write $r=\sin \psi$ for an appropriate $\psi$. To obtain a bound which is uniform in $\psi$, we use a trigonometric identity, the Cauchy-Schwarz inequality and change of variables to obtain

$$
\begin{aligned}
g(\sin \psi) & =\int_{0}^{2 \pi}\left(\frac{1+\sin \psi}{|\sin \psi+\sin \theta|}\right)^{\sigma / 2} \mathrm{~d} \theta=\int_{0}^{2 \pi}\left(\frac{1+\sin \psi}{\left|2 \sin \left(\frac{\psi+\theta}{2}\right) \cos \left(\frac{\psi-\theta}{2}\right)\right|}\right)^{\sigma / 2} \mathrm{~d} \theta \\
& \leq \int_{0}^{2 \pi}\left|\sin \left(\frac{\psi+\theta}{2}\right)\right|^{-\sigma / 2}\left|\cos \left(\frac{\psi-\theta}{2}\right)\right|^{-\sigma / 2} \mathrm{~d} \theta \\
& \leq\left(\int_{0}^{2 \pi}\left|\sin \left(\frac{\psi+\theta}{2}\right)\right|^{-\sigma} \mathrm{d} \theta\right)^{1 / 2}\left(\int_{0}^{2 \pi}\left|\cos \left(\frac{\psi-\theta}{2}\right)\right|^{-\sigma} \mathrm{d} \theta\right)^{1 / 2} \\
& =2 \int_{0}^{\pi}|\sin \theta|^{-\sigma} \mathrm{d} \theta .
\end{aligned}
$$

The latter integral is finite since $\sigma<1$. This completes the proof.

The lower bound in Theorem 1.4 follows by combining Proposition 4.7, Proposition 4.9 and Corollary 5.2. The almost sure upper bound for the vertical projections is the same as the universal upper bound which was proved in Proposition 4.2, and this bound is sharp.

Proposition 5.3. In each of the following statements, the set $A$ is a compact subset of $\mathbb{H}$.

(a) For all $0 \leq \beta \leq 1$ there exists $A$ such that

$$
\operatorname{dim}_{\mathbb{H}} A=\beta \text { and } \quad \operatorname{dim}_{\mathbb{H}} p_{\mathbb{W}_{\theta}}(A)=2 \beta \text { for all } \theta .
$$

(b) For all $1<\beta<3$ there exists $A$ such that

$$
\operatorname{dim}_{\mathbb{H}} A=\beta \quad \text { and } \quad \operatorname{dim}_{\mathbb{H}} p_{\mathbb{W}_{\theta}}(A)=\frac{1}{2}(\beta+3) \text { for all } \theta \text {. }
$$

(c) For all $3 \leq \beta \leq 4$ there exists $A$ such that

$$
\operatorname{dim}_{\mathbb{H}} A=\beta \text { and } \operatorname{dim}_{\mathbb{H}} p_{\mathbb{W}_{\theta}}(A)=3 \text { for all } \theta \text {. }
$$

Proof. First, we construct a set $A$ satisfying (a) for every $\theta$. Let $A \subset \mathbb{V}_{0}$ be a compact $\beta$-set. Then $p_{\mathbb{W}_{\theta}}(A)$ has Heisenberg Hausdorff dimension $2 \beta$ for every $\theta \neq 0$. Compare Example 4.1. To construct an example which works for every value of $\theta$, let $A$ be the union of two compact $\beta$-sets, one contained in $\mathbb{V}_{0}$ and one contained in $\mathbb{V}_{\pi / 2}$. 
It is not hard to find a set $A$ with

$$
\operatorname{dim}_{\mathbb{H}} A=\operatorname{dim}_{\mathbb{H}} p_{\mathbb{W}_{\theta}} A=3, \quad \text { for every } \theta \in[0, \pi)
$$

Take for instance

$$
A=\{(z, 0):|z| \leq 1\}
$$

The case (c) becomes then quite simple. Indeed, given $\beta \in[3,4]$, let $C \subset \mathbb{H}$ be any compact $\beta$-set which contains the set $A$. Then $p_{\mathbb{W}_{\theta}}(C) \supset p_{\mathbb{W}_{\theta}}(A)$ has dimension 3 for every $\theta$.

It remains to discuss the case (b). For a fixed number $\beta \in(1,3)$, we choose a Cantor set $C$ of Euclidean dimension $(\beta-1) / 2$ on the interval $[0,2 \pi)$ and set

$$
A:=\left\{\left(r e^{\mathrm{i} \varphi}, 0\right): r \in\left[\frac{1}{2}, 1\right], \varphi \in C\right\} .
$$

We will prove that $\operatorname{dim}_{\mathbb{H}} A=\beta$. The set $A$ is made up of horizontal curves, more precisely, radial segments inside the plane $t=0$. For almost every direction, the projection onto a vertical plane will be a non-horizontal parabola. This leads to the desired increase in dimension.

To define the set $C$, we employ the similarity maps $S_{1}(x)=\lambda x$ and $S_{2}(x)=$ $\lambda x+1-\lambda$ on $\mathbb{R}$ with

$$
\lambda:=4^{\frac{1}{1-\beta}} \in\left(0, \frac{1}{2}\right) .
$$

The resulting invariant set $C(\lambda)=S_{1}(C(\lambda)) \cup S_{2}(C(\lambda))$ is a compact subset of $[0,1]$ with $0<\mathcal{H}_{E}^{(\beta-1) / 2}(C(\lambda))<\infty$ and thus $\operatorname{dim}_{E} C(\lambda)=\frac{\log 2}{-\log \lambda}=\frac{\beta-1}{2}$. We set

$$
C:=\bigcup_{i=1}^{8} f_{i}(C(\lambda))
$$

where $f_{i}(\varphi)=\frac{\pi}{8} \varphi+(i-1) \frac{\pi}{4}$, and denote further

$$
A_{i}:=\left\{\left(r e^{\mathbf{i} \varphi}, 0\right): r \in\left[\frac{1}{2}, 1\right], \varphi \in f_{i}(C(\lambda))\right\} \quad \text { for } i \in\{1, \ldots, 8\} .
$$

Each set $A_{i}$ consists of radial segments of length $1 / 2$, emanating from a Cantor set on the unit circle.

The statement given in (b) then follows from the two subsequent lemmas:

Lemma 5.4. The set $A$ has dimension $\operatorname{dim}_{\mathbb{H}}(A)=\beta$.

Lemma 5.5. For an arbitrary $\theta \in[0, \pi)$, the set $p_{\mathbb{W}_{\theta}}(A)$ has dimension

$$
\operatorname{dim}_{\mathbb{H}}\left(p_{\mathbb{W}_{\theta}}(A)\right)=\frac{\beta+3}{2} .
$$

This finishes the proof of Proposition 5.3. 
Proof of Lemma 5.4. Proof of the upper bound. Fix $i \in\{1, \ldots, 8\}$. From $0<$ $\mathcal{H}_{E}^{(\beta-1) / 2}\left(f_{i}(C(\lambda))\right)<\infty$ it follows that

$$
0<\mathcal{H}_{E}^{\frac{\beta-1}{2}}\left(B_{i}\right)<\infty
$$

where $B_{i}:=\left\{\left(e^{\mathbf{i} \varphi}, 0\right): \varphi \in f_{i}(C(\lambda))\right\}$. Hence, for all $\varepsilon>0$ and $0 \leq \delta<1$, there exists a countable family of balls

$$
B_{E}\left(p_{n}, r_{n}\right), \quad n \in \mathbb{N} \text { with } r_{n} \leq \delta
$$

such that

$$
B_{i} \subseteq \bigcup_{n \in \mathbb{N}} B_{E}\left(p_{n}, r_{n}\right) \text { and } \sum_{n \in \mathbb{N}} r_{n}^{\frac{\beta-1}{2}+\frac{\varepsilon}{2}}<\delta .
$$

We may without loss of generality assume that the center $p_{n}=\left(e^{\mathbf{i} \varphi_{n}}, 0\right)$ lies on the unit circle in the plane. We will cover the segments

$$
\ell_{n}:=\left\{\left(r e^{\mathbf{i} \varphi_{n}}, 0\right): r \in\left[0, \frac{1}{2}\right]\right\}
$$

in an efficient way by small sets.

Let $p=\left(r e^{\mathbf{i} \varphi_{n}}, 0\right)$ be a point on $\ell_{n}$. Consider first the rectangle

$$
Q\left((r, 0), \sqrt{2 r_{n}}\right):=\left\{(z, 0)=(x+\mathbf{i} y, 0):|x-r| \leq \sqrt{2 r_{n}},|y| \leq 2 r_{n}\right\}
$$

in the plane centered at the point $(r, 0)$ on the $x$-axis. Rotate it to the point $p$ on $\ell_{n}$, i.e.,

$$
Q\left(p, \sqrt{2 r_{n}}\right)=\left\{\left(e^{\mathbf{i} \varphi_{n}} z, 0\right):(z, 0) \in Q\left((r, 0), \sqrt{2 r_{n}}\right)\right\}
$$

This set is contained in the Heisenberg ball $B_{\mathbb{H}}\left(p, c_{0} \sqrt{r_{n}}\right)$ for a constant $c_{0}>0$ which does not depend on $p$ or $r$.

We will cover the set $A_{i}$ by sets of the form $Q\left(p, \sqrt{2 r_{n}}\right)$ with $p \in \ell_{n}$. Recall that the line segment $\ell_{n}$ has length $1 / 2$ and each rectangle $Q\left(p, \sqrt{2 r_{n}}\right)$ centered on $\ell_{n}$ has length $\sqrt{2 r_{n}}$ in direction of $\ell_{n}$. It follows that there exist points $p_{n, 1}, \ldots, p_{n, N_{n}}$ on $\ell_{n}$ such that

$$
N_{n} \leq \frac{2}{\sqrt{r_{n}}} \quad \text { and } \quad \ell_{n} \subset \bigcup_{j=1}^{N_{n}} Q\left(p_{n, j}, \sqrt{2 r_{n}}\right) .
$$

We claim that

$$
\left\{Q\left(p_{n, j}, \sqrt{2 r_{n}}\right)\right\}_{j \in\left\{1, \ldots, N_{n}\right\}, n \in \mathbb{N}}
$$

covers the set $A_{i}$. To see this, let $p=\left(r e^{\mathbf{i} \varphi}, 0\right)$ be a point in $A_{i}$. Assume that $\varphi$ is different from all $\varphi_{n}, n \in \mathbb{N}$. The point $\left(e^{\mathbf{i} \varphi}, 0\right)$ lies in one of the balls $B_{E}\left(p_{n}, r_{n}\right)$ because they build a cover for the set $B_{i}$ on the unit circle. Hence, $p$ has distance at most $r_{n}$ from the line segment $\ell_{n}$ which is attached at the point $\left(e^{\mathbf{i} \varphi_{n}}, 0\right)$, thus it lies in one of the rectangles

$$
\left\{Q\left(p_{n, j}, \sqrt{2 r_{n}}\right)\right\}_{j \in\left\{1, \ldots, N_{j}\right\}}
$$


Since each of these rectangles is contained in a Heisenberg ball with the same center and radius $c_{0} \sqrt{r_{n}}$, it follows

$$
A_{i} \subseteq \bigcup_{n \in \mathbb{N}} \bigcup_{j=1}^{N_{n}} B_{\mathbb{H}}\left(p_{n, j}, c_{0} \sqrt{r_{n}}\right)
$$

with

$$
\begin{aligned}
\left(\mathcal{H}_{\mathbb{H}}\right)_{2 c_{0} \sqrt{\delta}}^{\beta+\varepsilon}\left(A_{i}\right) & \leq \sum_{n, j} \operatorname{diam}\left(B_{\mathbb{H}}\left(p_{n, j}, c_{0} \sqrt{r_{n}}\right)\right)^{\beta+\varepsilon}=\sum_{n \in \mathbb{N}} N_{n}\left(2 c_{0} \sqrt{r_{n}}\right)^{\beta+\varepsilon} \\
& \leq \sum_{n \in \mathbb{N}} \frac{2}{\sqrt{r_{n}}}\left(2 c_{0}\right)^{\beta+\varepsilon} r_{n}^{\frac{\beta+\varepsilon}{2}}=2\left(2 c_{0}\right)^{\beta+\varepsilon} \sum_{n \in \mathbb{N}} r_{n}^{\frac{\beta-1}{2}+\frac{\varepsilon}{2}}<\delta .
\end{aligned}
$$

Letting $\delta$ tend to zero, we see that $\mathcal{H}_{\mathbb{H}}^{\beta+\varepsilon}\left(A_{i}\right)=0$ for all $\varepsilon>0$ and thus

$$
\operatorname{dim}_{\mathbb{H}} A_{i} \leq \beta,
$$

which concludes the proof of the upper dimension bound in Lemma 5.4.

Proof of the lower bound. A lower bound for $\operatorname{dim}_{\mathbb{H}}(A)$ can be obtained from the mass distribution principle (Frostman's lemma). We have to find $r_{0}>0$ and a positive and finite measure $\mu$ on $A$ such that

$$
\mu\left(B_{\mathbb{H}}(p, r) \cap A\right) \leq r^{\beta} \quad \text { for all } p \in A, 0<r<r_{0} .
$$

For a Borel subset $E \subseteq A$, we define

$$
\mu(E):=\int_{C} \mathcal{H}_{E}^{1}\left(\ell_{\varphi} \cap E\right) \mathrm{d} \nu(\varphi),
$$

where $\nu$ is a Frostman measure on the $(\beta-1) / 2$-dimensional set $C$, that is, a positive and finite measure on $C$ with

$$
\nu((\varphi-r, \varphi+r) \cap C) \leq r^{\frac{\beta-1}{2}} \text { for all } \varphi \in C, r>0 .
$$

The set function $\mu$ is by definition positive and finite on $A$.

Since

$$
B_{\mathbb{H}}(p, r) \cap A \subseteq\left\{\left(\rho e^{\mathbf{i} \varphi}, 0\right):\left|\rho-\rho_{0}\right|<r,\left|\varphi-\varphi_{0}\right|<\pi r^{2}\right\}, \quad \text { for } p=\left(\rho_{0} e^{\mathbf{i} \varphi_{0}}, 0\right),
$$

it follows

$$
\mu\left(B_{\mathbb{H}}(p, r) \cap A\right) \leq r \cdot\left(\pi r^{2}\right)^{\frac{\beta-1}{2}}=\pi^{\frac{\beta-1}{2}} r^{\beta}
$$

for all $0<r<r_{0}$. An appropriate normalization of $\mu$ yields the desired Frostman measure to establish the lower bound for $\operatorname{dim}_{\mathbb{H}} A$.

In the subsequent discussion on $\operatorname{dim}_{\mathbb{H}} p_{\mathbb{W}_{\theta}}(A)$ we will again identify vertical planes in $\mathbb{H}$ with $\mathbb{R}^{2}$, so that a point $\left(\alpha \mathbf{i} e^{\mathbf{i} \theta}, \tau\right)$ in $\mathbb{W}_{\theta}$ is denoted by $(\alpha, \tau)$. 
Proof of Lemma 5.5. It is enough to prove

$$
\operatorname{dim}_{\mathbb{H}} p_{\mathbb{W}_{\theta}}(A) \geq(\beta+3) / 2 .
$$

This is again done by the mass distribution principle. Let us first explain how the set $p_{\mathbb{W}_{\theta}}(A)$ looks. For almost every direction $\theta \in[0, \pi)$, the line segment

$$
\ell_{\varphi}=\left\{\left(r e^{\mathbf{i} \varphi}, 0\right): r \in\left[\frac{1}{2}, 1\right]\right\}, \quad \varphi \in C,
$$

is mapped onto a parabola. Let us fix $\theta \in[0, \pi)$. We will prove that

$$
\operatorname{dim}_{\mathbb{H}} p_{\mathbb{W}_{\theta}}\left(A_{i}\right) \geq \frac{\beta+3}{2}
$$

for one of the subsets $A_{1}, \ldots, A_{8}$. This implies the lower bound for $\operatorname{dim}_{\mathbb{H}} p_{\mathbb{W}_{\theta}}(A)$. The reason why we work only with a subset of $A$ is that we can then ensure that there exists $\varepsilon>0$ such that

$$
\varphi-\theta \in\left[\varepsilon, \frac{\pi}{2}-\varepsilon\right] \text { for all } \varphi \in f_{i}(C(\lambda)),
$$

for an appropriate choice of $i \in\{1, \ldots, 8\}$, hence we can control the argument $\varphi-\theta$. This will be useful in the sequel. A direct computation shows that the set $p_{\mathbb{W}_{\theta}}\left(\ell_{\varphi}\right)$ coincides with the graph of the quadratic function

$$
f_{\varphi}: I_{\varphi} \rightarrow \mathbb{R}, \quad f_{\varphi}(\alpha)=-2 \cot (\varphi-\theta) \alpha^{2}
$$

with $I_{\varphi}=\left[\frac{1}{2} \sin (\varphi-\theta), \sin (\varphi-\theta)\right]$. Recall that we have chosen $i$ such that $\sin (\varphi-\theta)$ is positive and bounded away from 0 and 1 for all $\varphi \in f_{i}(C(\lambda))$.

We define a Frostman measure $\mu$ on $p_{\mathbb{W}_{\theta}}\left(A_{i}\right)$ as follows:

$$
\mu(E)=\int_{f_{i}(C(\lambda))} \mathcal{H}_{E}^{1}\left(p_{\mathbb{W}_{\theta}}\left(\ell_{\varphi}\right) \cap E\right) \mathrm{d} \nu(\varphi), \quad \text { for Borel sets } E \subseteq p_{\mathbb{W}_{\theta}}\left(A_{i}\right),
$$

where $\nu$ is a Frostman measure on $f_{i}(C(\lambda))$ which satisfies an upper mass bound with exponent $(\beta-1) / 2$. It is not hard to see that $\mu$ is positive and finite on $p_{\mathbb{W}_{\theta}}\left(A_{i}\right)$. To conclude the proof, it suffices to show that there exists $r_{0}>0$ such that

$$
\mu\left(B_{\mathbb{H}}(p, r) \cap p_{\mathbb{W}_{\theta}}\left(A_{i}\right)\right) \leq r^{\frac{\beta+3}{2}} \quad \text { for all } p \in p_{\mathbb{W}_{\theta}}\left(A_{i}\right) \text { and } 0<r<r_{0} \text {. }
$$

Let $p=(\alpha, \tau) \in p_{\mathbb{W}_{\theta}}\left(A_{i}\right)$. Since $B_{\mathbb{H}}(p, r) \cap p_{\mathbb{W}_{\theta}}\left(A_{i}\right)$ is a subset of $\mathbb{W}_{\theta}$, it is comparable to the rectangle

$$
R(p, r)=\left\{\left(\alpha^{\prime}, \tau^{\prime}\right):\left|\alpha-\alpha^{\prime}\right|<r,\left|\tau-\tau^{\prime}\right|<r^{2}\right\} .
$$

It is therefore enough to prove that

$$
\mu\left(R(p, r) \cap p_{\mathbb{W}_{\theta}}\left(A_{i}\right)\right) \leq r^{\frac{\beta+3}{2}} \quad \text { for all } p \in p_{\mathbb{W}_{\theta}}\left(A_{i}\right) \text { and } 0<r<r_{0} .
$$


To this end we estimate the measure $\mathcal{H}_{E}^{1}\left(p_{\mathbb{W}_{\theta}}\left(\ell_{\varphi}\right) \cap R(p, r)\right)$ for all $\varphi \in f_{i}(C(\lambda))$. Recall that $p_{\mathbb{W}_{\theta}}\left(\ell_{\varphi}\right)$ is the graph of the function $f_{\varphi}$ and therefore, if $p_{\mathbb{W}_{\theta}}\left(\ell_{\varphi}\right) \cap$ $R(p, r) \neq \emptyset$, we have

$$
\mathcal{H}_{E}^{1}\left(p_{\mathbb{W}_{\theta}}\left(\ell_{\varphi}\right) \cap R(p, r)\right)=\int_{\alpha_{1}}^{\alpha_{2}} \sqrt{1+\left(f_{\varphi}^{\prime}(\alpha)\right)^{2}} \mathrm{~d} \alpha \leq \sqrt{1+16 \cot ^{2}(\varepsilon)}\left(\alpha_{2}-\alpha_{1}\right),
$$

where $0<\alpha_{1}<\alpha_{2}<1$ are such that $f_{\varphi}\left(\alpha_{1}\right)=\tau+r^{2} / 2$ and $f_{\varphi}\left(\alpha_{2}\right)=\tau-r^{2} / 2$. We observe that

$$
r^{2}=2 \cot (\varphi-\theta)\left(\alpha_{2}^{2}-\alpha_{1}^{2}\right)
$$

and thus,

$$
\alpha_{2}-\alpha_{1}=\frac{r^{2}}{2 \cot (\varphi-\theta)\left(\alpha_{1}+\alpha_{2}\right)} \leq \frac{r^{2}}{2 \cot \left(\frac{\pi}{2}-\varepsilon\right) \sin (\varepsilon)} .
$$

Together, (5.7) and (5.8) imply that there exists a constant $c_{0}>0$, independent of $p, r$, and $\varphi$, such that

$$
\mathcal{H}_{E}^{1}\left(p_{\mathbb{W}_{\theta}}\left(\ell_{\varphi}\right) \cap R(p, r)\right) \leq c_{0} r^{2}
$$

Hence,

$$
\mu\left(R(p, r) \cap p_{\mathbb{W}_{\theta}}\left(A_{i}\right)\right) \leq c_{0} r^{2} \cdot \nu\left(\left\{\varphi \in f_{i}(C(\lambda)): p_{\mathbb{W}_{\theta}}\left(\ell_{\varphi}\right) \cap R(p, r) \neq \emptyset\right\}\right) .
$$

The parabolas $p_{\mathbb{W}_{\theta}}\left(\ell_{\varphi}\right)$ are graphs of functions of the type $g_{c}(\alpha)=c \alpha^{2}, \alpha \in \mathbb{R}$. A rectangle $R(p, r)$ in $\mathbb{R}^{2}$ with center $p=(\alpha, \tau)$ intersects the graph of $g_{c}$ only for particular values of $c$. If $p \in p_{\mathbb{W}_{\theta}}\left(A_{i}\right)$, it follows from the choice of $i$ that

$$
\alpha \geq \frac{1}{2} \sin (\varphi-\theta) \geq \frac{1}{2} \sin (\varepsilon)
$$

and

$$
\tau=-2 \cot (\varphi-\theta) \alpha^{2} \leq-\frac{1}{2} \cot \left(\frac{\pi}{2}-\varepsilon\right) \sin ^{2}(\varepsilon) .
$$

We choose now

$$
r_{0}:=\sqrt{\cot \left(\frac{\pi}{2}-\varepsilon\right) \sin ^{2}(\varepsilon)} .
$$

This ensures that $\tau+r^{2} / 2<0$ for $0<r<r_{0}$. The graph of $g_{c}$ crosses the rectangle $R(p, r)$ only if

$$
\frac{\tau-\frac{r^{2}}{2}}{\left(\alpha-\frac{r}{2}\right)^{2}} \leq c \leq \frac{\tau+\frac{r^{2}}{2}}{\left(\alpha+\frac{r}{2}\right)^{2}} .
$$

Here, we consider $g_{c}$ for $c=-2 \cot (\varphi-\theta)$ since the set $p_{\mathbb{W}_{\theta}}\left(\ell_{\varphi}\right)$ is the graph of $f_{\varphi}$. Hence, the parabola $p_{\mathbb{W}_{\theta}}\left(\ell_{\varphi}\right)$ intersects the rectangle $R(p, r)$ only if

$$
\varphi_{-}:=\cot ^{-1}\left(\frac{-\frac{\tau}{2}-\frac{r^{2}}{4}}{\left(\alpha+\frac{r}{2}\right)^{2}}\right)+\theta \leq \varphi \leq \cot ^{-1}\left(\frac{-\frac{\tau}{2}+\frac{r^{2}}{4}}{\left(\alpha-\frac{r}{2}\right)^{2}}\right)+\theta=: \varphi_{+} .
$$

It follows from the Mean Value Theorem that

$$
0 \leq \varphi_{+}-\varphi_{-} \leq \frac{1}{2} \frac{1}{\left(\alpha-\frac{r}{2}\right)^{2}\left(\alpha+\frac{r}{2}\right)^{2}}\left|-2 \alpha \tau r+\alpha^{2} r^{2}+\frac{r^{4}}{4}\right| .
$$


Notice that

$$
\alpha-\frac{r}{2} \geq \frac{1}{2} \sin (\varepsilon)-\frac{1}{2} \sqrt{\cot \left(\frac{\pi}{2}-\varepsilon\right) \sin ^{2}(\varepsilon)}=\frac{1}{2} \sin (\varepsilon)\left(1-\sqrt{\cot \left(\frac{\pi}{2}-\varepsilon\right)}\right) .
$$

We can choose $\varepsilon$ small enough such that the right-hand side is positive. Hence, there exists a constant $c_{1}>0$ such that

$$
\left|\varphi_{+}-\varphi_{-}\right| \leq c_{1} r
$$

Since

$$
\left\{\varphi \in f_{i}(C(\lambda)): p_{\mathbb{W}_{\theta}}\left(\ell_{\varphi}\right) \cap R(p, r) \neq \emptyset\right\} \subseteq\left(\varphi_{-}, \varphi_{+}\right)
$$

and $\nu$ is a measure on $f_{i}(C(\lambda))$ which satisfies an upper mass bound with exponent $(\beta-1) / 2$, we conclude

$$
\nu\left(\left\{\varphi \in f_{i}(C(\lambda)): p_{\mathbb{W}_{\theta}}\left(\ell_{\varphi}\right) \cap R(p, r) \neq \emptyset\right\}\right) \leq\left(\frac{c_{1}}{2}\right)^{\frac{\beta-1}{2}} r^{\frac{\beta-1}{2}}
$$

and thus, by (5.9), there follows

$$
\mu\left(R(p, r) \cap p_{\mathbb{W}_{\theta}}\left(A_{i}\right)\right) \leq c_{0} r^{2}\left(\frac{c_{1}}{2}\right)^{\frac{\beta-1}{2}} r^{\frac{\beta-1}{2}}=: c_{3} r^{\frac{\beta+3}{2}}
$$

for all $p \in p_{\mathbb{W}_{\theta}}\left(A_{i}\right)$ and $0<r<r_{0}$. This concludes the proof of Lemma 5.5.

\section{Projections of submanifolds}

In this section, we discuss first vertical projections of sets that possess a certain amount of regularity to substantiate the conjecture formulated in the introduction. In Proposition 6.1 we provide evidence for this conjecture. Note that sets satisfying the assumptions of Proposition 6.1 necessarily have positive Euclidean Hausdorff 2-measure. By the dimension comparison principle, sets $A \subset \mathbb{H}$ with $\operatorname{dim}_{\mathbb{H}} A>3$ also have positive Euclidean Hausdorff 2-measure. The conclusion in Proposition 6.1 is weaker than we would like. We do not know whether the projection must coincide with (or even contain) a continuous curve for at least one $\theta$.

Proposition 6.1. Let $A \subset \mathbb{H}$ be such that $\pi(A)=\Omega$ is a domain. If $p_{\mathbb{W}_{\theta}}(A)$ is the t-graph of a continuous function for a single value $\theta=\theta_{0}$, then $\mathcal{H}_{\mathbb{H}}^{3}\left(p_{\mathbb{W}_{\theta}}(A)\right)>0$ for every $\theta \neq \theta_{0}$.

Proof. As before, we begin with the representation (4.1) for the vertical projection. From the assumptions it follows that $A$ is the $t$-graph of a function $u$ over $\Omega$. Note that we do not assume that $u$ is continuous (although we will shortly see that in fact, $u$ must be continuous).

By performing a rotation if needed, we may assume that $\theta_{0}=0$. We compute

$$
\varphi_{\mathbb{W}_{0}} \circ p_{\mathbb{W}_{0}} \circ(\mathrm{id} \otimes u)(z)=\left(\operatorname{Im} z, u(z)-\operatorname{Im}\left(z^{2}\right)\right) .
$$


By assumption, this coincides with the graph map of a continuous function $h$. Thus

$$
u(z)=h(\operatorname{Im} z)+\operatorname{Im}\left(z^{2}\right)
$$

and so $u$ is in fact continuous. Furthermore, for any $\theta$,

$$
F_{\theta}(z):=\varphi_{\mathbb{W}_{\theta}} \circ p_{\mathbb{W}_{\theta}} \circ(\mathrm{id} \otimes u)(z)=\left(\operatorname{Im}\left(e^{-\mathbf{i} \theta} z\right), h(\operatorname{Im} z)+\operatorname{Im}\left(z^{2}\right)-\operatorname{Im}\left(e^{-2 \mathbf{i} \theta} z^{2}\right)\right) .
$$

We claim that $F_{\theta}(\Omega)$ has positive area for any $\theta \neq 0$. By Fubini's theorem,

$$
\mathcal{H}_{E}^{2}\left(F_{\theta}(\Omega)\right)=\int \mathcal{H}_{E}^{1}\left((\{a\} \times \mathbb{R}) \cap F_{\theta}(\Omega)\right) d a .
$$

Let us observe that a point $(a, t)$ lies in $F_{\theta}(\Omega)$ if and only if the following conditions hold:

$$
a=\operatorname{Im}\left(e^{-\mathbf{i} \theta} z\right) \quad \text { and } \quad t=h(\operatorname{Im} z)+\operatorname{Im}\left(z^{2}\right)-\operatorname{Im}\left(e^{-2 \mathbf{i} \theta} z^{2}\right)
$$

for some $z \in \Omega$.

Assume that $0<\theta<\pi$ and write $z=x+\mathbf{i} y$. Then

$$
a=y \cos \theta-x \sin \theta
$$

and

$$
t=h(y)+2 x y-2(y \cos \theta-x \sin \theta)(x \cos \theta+y \sin \theta) .
$$

Substituting (6.2) into (6.3) yields

$$
t=h(y)+(2 \cot \theta) y^{2}-(4 \csc \theta) a y+(2 \cot \theta) a^{2}=: h(y)+Q_{\theta, a}(y) .
$$

For a positive $\mathcal{H}_{E}^{1}$ measure set of values of the variable $a$, the integrand in (6.1) is equal to the $\mathcal{H}_{E}^{1}$ measure of the image of

$$
\left\{y:\left(\frac{y \cos \theta-a}{\sin \theta}, y\right) \in \Omega\right\}
$$

under the map $h+Q_{\theta, a}$. The set in (6.4) is a union of open intervals, and the map $h+Q_{\theta, a}$ is continuous, hence the integrand in (6.1) is strictly positive for a positive $\mathcal{H}_{E}^{1}$ measure set of values of $a$. Consequently,

$$
\mathcal{H}_{E}^{2}\left(F_{\theta}(\Omega)\right)>0
$$

This completes the proof.

Similarly as in Proposition 6.1, we consider in the following the effect of vertical projections on subsets with additional regularity assumptions. This allows a more precise statement than in the general case of arbitrary Borel subset.

Theorem 6.2. For any $C^{1}$ curve $\gamma$ in $\mathbb{H}$, the value of $\operatorname{dim}_{\mathbb{H}} p_{\mathbb{W}_{\theta}} \gamma$ can be equal to 0 or 1 for at most two values of $\theta$, and is equal to 2 for all other values of $\theta$. 
Theorem 6.3. For any $C^{1}$ surface $\Sigma$ in $\mathbb{H}$, the value of $\operatorname{dim}_{\mathbb{H}} p_{\mathbb{W}_{\theta}} \Sigma$ can be equal to 1 or 2 for at most one value of $\theta$, and is equal to 3 for all other values of $\theta$.

Note that $\operatorname{dim}_{\mathbb{H}} \gamma$ can be equal to either 1 or 2 for a $C^{1}$ curve $\gamma$, depending on whether or not $\gamma$ is a horizontal curve. However, $\operatorname{dim}_{\mathbb{H}} \Sigma$ is equal to 3 for all $C^{1}$ surfaces $\Sigma$. See, for example, Section 0.6.C in [11].

Recall also that the restriction of the Heisenberg metric to $\mathbb{W}_{\theta}$ is comparable with the heat metric; see (1.4).

A $C^{1}$ curve $\gamma$ which lies in $\mathbb{W}_{\theta}$ is horizontal (as a curve in $\mathbb{H}$ ) if and only if it is contained in a horizontal line. In other words, if $\gamma \subset \mathbb{W}_{\theta}$ for some $\theta$, then $\gamma^{\prime} \in H_{\gamma} \mathbb{H}$ if and only if $\gamma \subset\{t=c\}$ for some $c$.

For $\theta \in[0, \pi)$ and $c \in \mathbb{R}$, let us define

$$
\Sigma_{\theta, c}:=p_{\mathbb{W}_{\theta}}^{-1}\left(\{t=c\} \cap \mathbb{W}_{\theta}\right) .
$$

Note that $\Sigma_{\theta, c}$ consists of points in $\mathbb{H}$ of the form $\left((r+\mathbf{i} a) e^{\mathbf{i} \theta}, c+2 a r\right)$, where $a, r \in \mathbb{R}$. Equivalently, $\Sigma_{\theta, c}$ is the graph of the function $t=\varphi_{\theta, c}(x, y)$ given by

$$
\varphi_{\theta, c}(x, y)=2(x \cos \theta+y \sin \theta)(y \cos \theta-x \sin \theta)+c .
$$

From the preceding remarks we observe:

Proposition 6.4. (1) Let $\gamma$ be a $C^{1}$ curve in $\mathbb{H}$. Then $p_{\mathbb{W}_{\theta}}(\gamma)$ is horizontal if and only if there exists $c$ so that $\gamma \subset \Sigma_{\theta, c}$.

(2) Let $\Sigma$ be a $C^{1}$ surface in $\mathbb{H}$. Then $p_{\mathbb{W}_{\theta}}(\Sigma)$ is horizontal if and only if there exists $c$ so that $\Sigma \subset \Sigma_{\theta, c}$.

We also need a lemma on the intersection properties of the surfaces $\Sigma_{\theta, c}$.

Lemma 6.5. (1) Let $\left(\theta_{1}, c_{1}\right)$ and $\left(\theta_{2}, c_{2}\right)$ be distinct, $c_{1}, c_{2} \in \mathbb{R}$. If $\theta_{1} \neq \theta_{2}$, then $\Sigma_{\theta_{1}, c_{1}} \cap \Sigma_{\theta_{2}, c_{2}}$ is a $C^{1}$ curve. If $\theta_{1}=\theta_{2}$ and $c_{1} \neq c_{2}$, then $\Sigma_{\theta_{1}, c_{1}} \cap \Sigma_{\theta_{2}, c_{2}}$ is empty.

(2) Let $\left(\theta_{1}, c_{1}\right),\left(\theta_{2}, c_{2}\right)$ and $\left(\theta_{3}, c_{3}\right)$ be pairwise distinct. If the $\theta_{i}$ 's are all pairwise distinct, then $\Sigma_{\theta_{1}, c_{1}} \cap \Sigma_{\theta_{2}, c_{2}} \cap \Sigma_{\theta_{3}, c_{3}}$ is a point. If exactly two of the $\theta_{i}$ 's are equal, then $\Sigma_{\theta_{1}, c_{1}} \cap \Sigma_{\theta_{2}, c_{2}} \cap \Sigma_{\theta_{3}, c_{3}}$ is a $C^{1}$ curve. If $\theta_{1}=\theta_{2}=\theta_{3}$, then $\Sigma_{\theta_{1}, c_{1}} \cap \Sigma_{\theta_{2}, c_{2}} \cap \Sigma_{\theta_{3}, c_{3}}$ is empty.

Proof of Theorems 6.2 and 6.3. If $\gamma$ is a $C^{1}$ curve in $\mathbb{H}$, then $p_{\mathbb{W}_{\theta}} \gamma$ is either a $C^{1}$ curve or a point in $\mathbb{W}_{\theta}$. If it is a curve, then its dimension is either equal to 1 or equal to 2, depending on whether or not the curve is horizontal. By the proposition, this curve is horizontal if and only if $\gamma$ is contained in $\Sigma_{\theta, c}$ for some $c$. By the lemma, at most two distinct surfaces of this type can intersect along a $C^{1}$ curve. This completes the proof of Theorem 6.2.

Now suppose that $\Sigma$ is a $C^{1}$ surface in $\mathbb{H}$. Then $p_{\mathbb{W}_{\theta}}(\Sigma)$ is either a $C^{1}$ surface, a $C^{1}$ curve or a point in $\mathbb{W}_{\theta}$. The rest of the argument is similar to the one in the previous paragraph. 


\section{Projections of subsets of horizontal or vertical planes}

In this section, we discuss methods to improve the lower dimensional bounds for vertical projections. Energy integrals can be used to obtain better lower bounds for sets of dimension at most two lying inside a horizontal plane, or for sets of dimension at least one lying inside a vertical plane.

Recall that in order to obtain lower bounds for the dimension of projections, the goal was to ascertain that the integral

$$
\int_{G(n, m)} I_{s}\left(\left(P_{V}\right)_{\sharp} \mu\right) d \gamma_{n, m}(V),
$$

respectively $\int_{0}^{\pi} I_{s}\left(\left(p_{\mathbb{W}_{\theta}}\right)_{\sharp} \mu\right) \mathrm{d} \theta$ in the Heisenberg case, was finite for any given $s<\operatorname{dim} A$ and $\mu \in \mathcal{M}(A)$ with $I_{s}(\mu)<\infty$. To obtain the finiteness of an integral as in (7.1) in the Euclidean case, one shows that

$$
\int_{G(n, m)} d\left(P_{V}(p), P_{V}(q)\right)^{-s} d \gamma_{n, m}(V) \leq c d(p, q)^{-s},
$$

and uses the finiteness of $I_{s}(\mu)$.

In this section, we establish estimates of the type (7.2) for Heisenberg vertical projections and apply them to get dimension bounds for vertical projections of subsets of horizontal or vertical planes. In Subsection 7.1 such a result is proved for points in a horizontal plane when $s<2$. Moreover, we show that this pointwise bound does not hold in general for larger $s$. In Subsection 7.2 we establish a similar result for vertical planes with different exponents on the two sides of the inequality (7.2).

It is not hard to see that one cannot in general get a pointwise estimate of the form (7.2). But one could hope that the set of points $(p, q)$ where this bound does not hold is small with respect to the measure $\mu$. In that case, one could anticipate proving the finiteness of an integral of the type (7.1). In Subsection 7.3 we show that this hope is vain. We give examples where the integral is infinite, even in case the projections are known to be of dimension at least $s$.

We use the following notation. For a pair of functions $f, g: A \rightarrow[0, \infty]$ we write

$$
f(p) \simeq g(p)
$$

if there exist constants $c_{0}, c_{1}>0$ such that

$$
c_{0} f(p) \leq g(p) \leq c_{1} f(p) \text { for all } p \in A
$$

If only one of the two inequalities hold, we write accordingly

$$
f(p) \lesssim g(p) \text { or } \quad f(p) \gtrsim g(p) .
$$

We denote by $p=(z, t)$ and $q=(\zeta, \tau)$ points in $\mathbb{H}$ and use the following abbreviated notation:

$$
\varphi_{1}=\arg (z-\zeta), \quad \varphi_{2}=\arg (z+\zeta)
$$


In the proofs of the dimension theorems, one works with integrals of the form

$$
\begin{aligned}
& J_{s}(p, q)=\int_{0}^{\pi} d_{\mathbb{H}}\left(p_{\mathbb{W}_{\theta}}(p), p_{\mathbb{W}_{\theta}}(q)\right)^{-s} \mathrm{~d} \theta \\
& (7.6)=\int_{0}^{\pi}\left(|z-\zeta|^{4} \sin ^{4}\left(\varphi_{1}-\theta\right)+\left(t-\tau-\left|z^{2}-\zeta^{2}\right| \sin \left(\varphi_{1}+\varphi_{2}-2 \theta\right)\right)^{2}\right)^{-s / 4} \mathrm{~d} \theta .
\end{aligned}
$$

(The arguments $\varphi_{1}$ and $\varphi_{2}$ are not well defined for $z-\zeta=0$ or $z+\zeta=0$, but in this case also $|z-\zeta|=0$ or $\left|z^{2}-\zeta^{2}\right|=0$, which will ensure that the respective terms vanish.)

The following result will be applied several times. We skip the easy proof.

Lemma 7.1. Let $p_{0}=\left(z_{0}, t_{0}\right)$ be a point in $\mathbb{H}$ with $\left|z_{0}\right|=R>0$. Then, for all $0<r<R / 2$ and all points $p=(z, t)$ and $q=(\zeta, \tau)$ in $B_{\mathbb{H}}\left(p_{0}, r\right)$, the distance $|z-\zeta|$ is comparable to $\left|z^{2}-\zeta^{2}\right|$.

\subsection{Dimension estimates for sets lying in a horizontal plane}

In this section, the dimension parameter $s$ will be fixed. All implicit constants in relations of type (7.3) or (7.4) are allowed to depend on $s$, but are independent of all other parameters or variables.

Proposition 7.2. Assume that $0<s<2$. Let $z_{0} \in \mathbb{C}, z_{0} \neq 0$, and let $t_{0} \in \mathbb{R}$. Then

$$
J_{s}(p, q) \lesssim d_{\mathbb{H}}(p, q)^{-s}
$$

for points $p$ and $q$ in $\left\{p=(z, t) \in \mathbb{H}: t=t_{0}\right\} \cap B_{\mathbb{H}}\left(\left(z_{0}, t_{0}\right), \frac{1}{20}\left|z_{0}\right|\right)$.

Proof. By applying a preliminary dilation, we may assume without loss of generality that $\left|z_{0}\right|=1$. Let $p=\left(z, t_{0}\right)$ and $q=\left(\zeta, t_{0}\right)$ be distinct points in $B_{\mathbb{H}}\left(p_{0}, \frac{1}{20}\right)$, where $p_{0}=\left(z_{0}, t_{0}\right)$. By Lemma 7.1, we have

$$
\left|z^{2}-\zeta^{2}\right|=|z-\zeta||z+\zeta| \simeq|z-\zeta|=: a .
$$

Note that

$$
0<a=|z-\zeta| \leq\left|z-z_{0}\right|+\left|z_{0}-\zeta\right| \leq d_{\mathbb{H}}\left(p, p_{0}\right)+d_{\mathbb{H}}\left(p_{0}, q\right)<\frac{1}{10} .
$$

By Lemma 7.1 and substituting $\psi=2 \varphi_{1}-2 \theta$, we have

$$
J_{s}(p, q) \simeq \int_{0}^{2 \pi}\left(a^{4} \sin ^{4}\left(\frac{\psi}{2}\right)+a^{2} \sin ^{2}(\psi-\alpha)\right)^{-s / 4} \mathrm{~d} \psi,
$$

with $\alpha=\varphi_{1}-\varphi_{2}+k \pi$, where $k \in \mathbb{N}$ is chosen so that $\alpha$ lies in $[0, \pi)$.

Using some elementary estimates for the sine function, we conclude that

$$
\int_{0}^{2 \pi}\left(a^{4} \sin ^{4} \frac{\psi}{2}+a^{2} \sin ^{2}(\psi-\alpha)\right)^{-s / 4} \mathrm{~d} \psi \simeq a^{-s} \int_{0}^{2 \pi} h(\psi)^{-s / 4} \mathrm{~d} \psi
$$


for $\alpha \in[0, \pi / 2)$, where

$$
h(\psi)=\min \{\psi, 2 \pi-\psi\}^{4}+\left(\frac{\min \{|\psi-\alpha|,|\psi-\alpha-\pi|,|\psi-\alpha-2 \pi|\}}{a}\right)^{2} .
$$

We split the integral on the right-hand side of (7.9) into four terms, integrating over the intervals $[0, \pi / 2+\alpha],[\pi / 2+\alpha, \pi],[\pi, 3 \pi / 2+\alpha]$ and $[3 \pi / 2+\alpha, 2 \pi]$ in turn. In each of the resulting integrals, we perform a linear change of variables to rewrite the integral as an integral over the interval $[0,1]$. For instance, the substitution $x=c^{-1} \psi$ with $c=\alpha+\pi / 2 \in[\pi / 2, \pi)$ in the first term yields

$$
a^{-s} \int_{0}^{\alpha+\pi / 2}\left(\psi^{4}+\left(\frac{\psi-\alpha}{a}\right)^{2}\right)^{-s / 4} \mathrm{~d} \psi \simeq a^{-s} \int_{0}^{1}\left(x^{4}+\left(\frac{x-\beta}{a}\right)^{2}\right)^{-s / 4} \mathrm{~d} x,
$$

with $\beta=\frac{\alpha}{\alpha+\pi / 2} \in[0,1 / 2]$. Each of the remaining three integrals is dominated by the integral on the right hand side of (7.10). This is easily seen by evaluating separately each integral.

If $\alpha \in[\pi / 2, \pi]$, we see in the same way that

$$
\begin{aligned}
J_{s}(p, q) & \simeq a^{-s} \int_{\alpha+\frac{\pi}{2}}^{2 \pi}\left((\psi-2 \pi)^{4}+\left(\frac{\psi-\alpha-\pi}{a}\right)^{2}\right)^{-s / 4} \mathrm{~d} \psi \\
& \simeq a^{-s} \int_{0}^{1}\left(x^{4}+\left(\frac{x-\beta}{a}\right)^{2}\right)^{-s / 4} \mathrm{~d} x,
\end{aligned}
$$

with $\beta=\frac{\pi-\alpha}{(3 \pi / 2)-\alpha} \in[0,1 / 2]$.

Let us consider integrals of the type $\int_{0}^{1}\left(x^{4}+\left(\frac{x-\beta}{a}\right)^{2}\right)^{-s / 4} \mathrm{~d} x$ with $\beta \in[0,1 / 2]$ and $a \in(0,1)$.

Lemma 7.3. For $0<s<2, \beta \in[0,1 / 2]$ and $a \in(0,1)$, we have

$$
\int_{0}^{1}\left(x^{4}+\left(\frac{x-\beta}{a}\right)^{2}\right)^{-s / 4} \mathrm{~d} x \simeq a^{s / 2} .
$$

Proof of Lemma 7.3. First, assume that $\beta>0$. We integrate over intervals where one of the summands is dominating. Fix $\delta=a \beta^{2}$ and note that $\delta \leq \beta / 2$. Let $I$ denote the integral in (7.12). We split the region of integration into three subregions, integrating over $[0, \beta-\delta],[\beta-\delta, \beta+\delta]$ and $[\beta+\delta, 1]$, respectively. By some elementary calculations we find

$$
\begin{aligned}
I & \simeq \int_{0}^{\beta-\delta}\left(\frac{\beta-x}{a}\right)^{-s / 2} \mathrm{~d} x+\int_{\beta-\delta}^{\beta+\delta} x^{-s} \mathrm{~d} x+\int_{\beta+\delta}^{1}\left(\frac{x-\beta}{a}\right)^{-s / 2} \mathrm{~d} x \\
& \simeq a^{s / 2}\left(\beta^{1-s / 2}-\delta^{1-s / 2}\right)+\int_{\beta-\delta}^{\beta+\delta} x^{-s} \mathrm{~d} x+a^{s / 2}\left((1-\beta)^{1-s / 2}-\delta^{1-s / 2}\right) .
\end{aligned}
$$

By the Mean Value Theorem, $\int_{\beta-\delta}^{\beta+\delta} x^{-s} \mathrm{~d} x=2 \delta(1-s) \xi^{-s}$ for some $\xi$ in $[\beta-\delta, \beta+\delta]$. Further calculations yield

$$
I \simeq a^{s / 2}\left(\beta^{1-s / 2}+(1-\beta)^{1-s / 2}\right)+\delta \beta^{-s}=a^{s / 2}\left(\beta^{1-s / 2}+(1-\beta)^{1-s / 2}\right)+a \beta^{2-s} .
$$

Since $s<2$ and $a, \beta<1$ we conclude that $I \simeq a^{s / 2}$ as desired. 
The case $\beta=0$ is even simpler. In this case,

$$
I=\int_{0}^{1}\left(x^{4}+\frac{x^{2}}{a^{2}}\right)^{-s / 4} \mathrm{~d} x \simeq a^{s / 2} \int_{0}^{1} x^{-s / 2} \mathrm{~d} x \simeq a^{s / 2} .
$$

This completes the proof of the lemma.

In view of Lemma 7.3, (7.11) implies that

$$
J_{s}(p, q) \simeq a^{-s / 2}=|z-\zeta|^{-s / 2} .
$$

Our next goal is to compare this last expression to the distance between the two points $p$ and $q$. To this end, note that

$$
d_{\mathbb{H}}(p, q)^{4} \simeq a^{4}+a^{2} \sin ^{2}\left(\varphi_{1}-\varphi_{2}\right) \leq 2 a^{2} .
$$

and therefore

$$
a^{-s / 2} \lesssim d_{\mathbb{H}}(p, q)^{-s}
$$

Combining (7.15) and (7.16) completes the proof of Proposition 7.2.

Remark 7.4. A statement analogous to Proposition 7.2 does not hold for $s>2$. Let us observe that the estimate in (7.14) holds for all $s>0$ and $\beta>0$; the implicit constants depend on $s$ but not on $\beta$. Keeping only the final term yields

$$
I \gtrsim a \beta^{2-s} \text {. }
$$

Recall that $\beta \simeq \alpha$ if $0 \leq \alpha \leq \pi / 2$ while $\beta \simeq \pi-\alpha$ if $\pi / 2 \leq \alpha \leq \pi$. Put simply, $\beta \simeq \sin \alpha$ and consequently,

$$
J_{s}(p, q)=a^{-s} I \gtrsim a^{1-s}(\sin \alpha)^{2-s} .
$$

If (7.7) were true, it would then imply

$$
a^{1-s}(\sin \alpha)^{2-s} \lesssim\left(a^{4}+a^{2} \sin ^{2} \alpha\right)^{-s / 4}
$$

The latter statement is true if and only if

$$
a^{2}+\sin ^{2} \alpha \lesssim a^{2(s-2) / s}(\sin \alpha)^{4(s-2) / s},
$$

however, it is clear that (7.17) is impossible if $a$ is either much smaller or much larger than $\sin \alpha$. This shows that the estimate

$$
J_{s}(p, q) \lesssim d_{\mathbb{H}}(p, q)^{-s}
$$

cannot hold on $B_{\mathbb{H}}\left(1, \frac{1}{20}\right) \cap\left\{(z, t) \in \mathbb{H}: t=t_{0}\right\}$ if $s>2$ for any $t_{0} \in \mathbb{R}$.

Proposition 7.2 can be applied to obtain an almost sure lower dimension bound for subsets in a horizontal plane of Hausdorff dimension at most 2.

Proposition 7.5. Let $A$ be a Borel set lying inside a horizontal plane $\{(z, t) \in \mathbb{H}$ : $\left.t=t_{0}\right\}$. If $\operatorname{dim}_{\mathbb{H}} A \leq 2$, then $\operatorname{dim}_{\mathbb{H}} p_{\mathbb{W}_{\theta}}(A) \geq \operatorname{dim}_{\mathbb{H}} A$ for almost every $\theta \in[0, \pi)$. 
Proof. In view of Theorem 1.4, we may assume without loss of generality that $\operatorname{dim}_{\mathbb{H}} A>1$. We may also assume that $A \subset B_{\mathbb{H}}\left(p_{0}, r\right)$ for some $p_{0}=\left(z_{0}, t_{0}\right)$ with $r<\frac{1}{20}\left|z_{0}\right|$, since $\mathbb{H} \backslash\{z=0\}$ can be covered with countably many such balls. Let $s<\operatorname{dim}_{\mathbb{H}} A$. By the energy version of Frostman's lemma, there exists $\mu \in \mathcal{M}(A)$ with $I_{s}(\mu)<\infty$. Then

$$
\begin{aligned}
\int_{0}^{\pi} I_{s}\left(\left(p_{\mathbb{W}_{\theta}}\right)_{\sharp} \mu\right) \mathrm{d} \theta & =\int_{A} \int_{A} \int_{0}^{\pi} \frac{1}{d_{\mathbb{H}}\left(p_{\mathbb{W}_{\theta}}(p), p_{\mathbb{W}_{\theta}}(q)\right)^{s}} \mathrm{~d} \theta \mathrm{d} \mu(p) \mathrm{d} \mu(q) \\
& =\int_{A} \int_{A} J_{s}(p, q) \mathrm{d} \mu(p) \mathrm{d} \mu(q),
\end{aligned}
$$

where $J_{s}(p, q)$ is as in (7.6). Since $A$ is a subset of $B_{\mathbb{H}}\left(p_{0}, r\right)$ inside the horizontal plane $\left\{t=t_{0}\right\}$, we can apply Proposition 7.2 to obtain

$$
J_{s}(p, q) \lesssim d_{\mathbb{H}}(p, q)^{-s}
$$

Then

$$
\int_{0}^{\pi} I_{s}\left(\left(p_{\mathbb{W}_{\theta}}\right)_{\sharp} \mu\right) \mathrm{d} \theta \lesssim \int_{A} \int_{A} d_{\mathbb{H}}(p, q)^{-s} \mathrm{~d} \mu(p) \mathrm{d} \mu(q)=I_{s}(\mu)
$$

is finite, which implies that $I_{s}\left(\left(p_{\mathbb{W}_{\theta}}\right)_{\sharp} \mu\right)<\infty$ and thus $\operatorname{dim}_{\mathbb{H}} p_{\mathbb{W}_{\theta}}(A) \geq s$ for almost every $\theta \in[0, \pi)$. Letting $s$ tend to $\operatorname{dim}_{\mathbb{H}} A$ gives the desired conclusion.

Remark 7.6. An analogous proof can be used to show that a set $A$ inside a horizontal plane $\left\{(z, t) \in \mathbb{H}: t=t_{0}\right\}$ with $\operatorname{dim}_{E} A \leq 1$ has

$$
\operatorname{dim}_{\mathbb{H}} p_{\mathbb{W}_{\theta}}(A) \geq 2 \operatorname{dim}_{E} A \text { for almost every } \theta \in[0, \pi) .
$$

One applies the intermediate result (7.15) instead of the final result of Proposition 7.2. In view of the upper bound (4.3), we conclude from (7.19) that

$$
\operatorname{dim}_{\mathbb{H}} p_{\mathbb{W}_{\theta}}(A)=2 \operatorname{dim}_{E} A \text { for almost every } \theta \in[0, \pi)
$$

whenever $A$ is a subset of a horizontal plane with $\operatorname{dim}_{E} A \leq 1$.

Let us briefly indicate why (7.19) holds. To avoid confusion, we denote by $I_{s}^{E}(\mu)$ the $s$-energy of a measure $\mu$ computed with respect to the Euclidean metric and by $I_{s}^{\mathbb{H}}(\mu)$ the $s$-energy of $\mu$ computed with respect to the Heisenberg metric. Let $A$ be a subset of a horizontal plane with $\operatorname{dim}_{E} A \leq 1$. We may assume without loss of generality that $A$ is contained inside an appropriate ball so that the conclusion of Proposition 7.2 is valid for points $p=(z, t)$ and $q=(\zeta, \tau)$ in $A$.

Let $s<2 \operatorname{dim}_{E} A$ be arbitrary. Then $s<2$ and so (7.15) is valid for this value of $s$. Choose a measure $\mu$ on $A$ so that $I_{s / 2}^{E}(\mu)$ is finite. We conclude that

$$
\begin{aligned}
\int_{0}^{\pi} I_{s}^{\mathbb{H}}\left(\left(p_{\mathbb{W}_{\theta}}\right)_{\sharp} \mu\right) \mathrm{d} \theta & =\int_{A} \int_{A} \int_{0}^{\pi} d_{\mathbb{H}}\left(p_{\mathbb{W}_{\theta}}(p), p_{\mathbb{W}_{\theta}}(q)\right)^{-s} \mathrm{~d} \theta d \mu(p) \mathrm{d} \mu(q) \\
& =\int_{A} \int_{A} J_{s}(p, q) d \mu(p) \mathrm{d} \mu(q) \simeq \int_{A} \int_{A}|z-\zeta|^{-s / 2} d \mu(p) \mathrm{d} \mu(q) \\
& =\int_{A} \int_{A} d_{E}(p, q)^{-s / 2} d \mu(p) \mathrm{d} \mu(q)=I_{s / 2}^{E}(\mu)
\end{aligned}
$$


is finite. Here we used the fact that $d_{E}(p, q)=|z-\zeta|$ for points $p=(z, t)$ and $q=(\zeta, \tau)$ lying in a horizontal plane. Thus $I_{s}^{\mathbb{H}}\left(\left(p_{\mathbb{W}_{\theta}}\right)_{\sharp} \mu\right)$ is finite for almost every $\theta$ and so $\operatorname{dim}_{\mathbb{H}}\left(p_{\mathbb{W}_{\theta}}(A)\right) \geq s$ for almost every $\theta$. Letting $s$ increase to $2 \operatorname{dim}_{\mathbb{H}} A$ completes the proof.

\subsection{Dimension estimates for sets lying in a vertical plane}

In this section we consider sets which lie inside a vertical plane $\mathbb{W}_{\theta_{0}}$. We will study integrals $J_{s}(p, q)$ for distinct points $p, q \in W_{\theta_{0}}$ and $s \in(1,2)$. Let us assume further that $p$ and $q$ are contained in a ball of the form

$$
B_{\mathbb{H}}\left(\left(\mathbf{i} e^{\mathbf{i} \theta_{0}}, 0\right), \frac{1}{20}\right) .
$$

As before, this is not a restrictive assumption.

We will prove:

Proposition 7.7. Assume that $1<s<2$ and let

$$
\sigma=2 s-1
$$

Let $\theta_{0} \in[0,2 \pi]$. Then

$$
J_{s}(p, q) \lesssim d_{\mathbb{H}}(p, q)^{-\sigma}
$$

for points $p, q \in W_{\theta_{0}} \cap B_{\mathbb{H}}\left(\left(\mathbf{i} e^{\mathbf{i} \theta_{0}}, 0\right), \frac{1}{20}\right)$.

Note that $1<s<\sigma<3$.

Proof. Let $p=(z, t)$ and $q=(\zeta, \tau)$ as before. From

$$
\left(|z-\zeta|^{4}+(t-\tau)^{2}\right)^{1 / 4}=d_{\mathbb{H}}(p, q) \leq d_{\mathbb{H}}\left(p,\left(\mathbf{i} e^{\mathbf{i} \theta_{0}}, 0\right)\right)+d_{\mathbb{H}}\left(\left(\mathbf{i} e^{\mathbf{i} \theta_{0}}, 0\right), q\right)<\frac{1}{10}
$$

it follows that

$$
|z-\zeta|<\frac{1}{10} \quad \text { and } \quad|t-\tau|<\frac{1}{100}
$$

By reordering the points $p$ and $q$ if necessary, we may without loss of generality assume that $t-\tau \geq 0$. We denote

$$
A:=\left|z^{2}-\zeta^{2}\right|=|z-\zeta||z+\zeta| \text { and } B:=t-\tau,
$$

and observe that $A \simeq|z-\zeta|$, see Lemma 7.1. The statement for $A=0$ is trivial, we will therefore from now on assume that $A \neq 0$.

We note that the two angles $\varphi_{1}=\arg (z-\zeta)=\arg \left((|z|-|\zeta|) \mathbf{i} e^{\mathbf{i} \theta_{0}}\right)$ and $\varphi_{2}=$ $\arg (z+\zeta)=\arg \left((|z|+|\zeta|) \mathbf{i} e^{\mathbf{i} \theta_{0}}\right)$ coincide if $|z| \geq|\zeta|$ and differ by $\pi$ if $|z|<|\zeta|$. Then for all $\theta \in[0, \pi)$ we find

$$
\begin{aligned}
d_{\mathbb{H}}\left(p_{\mathbb{W}_{\theta}}(p), p_{\mathbb{W}_{\theta}}(q)\right)^{4} & =|z-\zeta|^{4} \sin ^{4}\left(\varphi_{1}-\theta\right)+\left(B \pm A \sin \left(2\left(\varphi_{1}-\theta\right)\right)\right)^{2} \\
& \simeq A^{4} \sin ^{4}\left(\varphi_{1}-\theta\right)+\left(B \pm A \sin \left(2\left(\varphi_{1}-\theta\right)\right)\right)^{2}
\end{aligned}
$$


where the choice of the sign coincides with the sign of $|\zeta|-|z|$. Substituting $\psi=2\left(\varphi_{1}-\theta\right)$ or $\psi=-2\left(\varphi_{1}-\theta\right)$ as appropriate, we conclude in either case that

$$
J_{s}(p, q) \simeq \int_{0}^{2 \pi}\left(A^{4} \sin ^{4}\left(\frac{\psi}{2}\right)+(B-A \sin \psi)^{2}\right)^{-s / 4} \mathrm{~d} \psi .
$$

For some $\alpha_{0} \in\{0, \pi / 4, \ldots, 7 \pi / 4\}$ we have

$$
\begin{aligned}
J_{s}(p, q) & \simeq \int_{\alpha_{0}}^{\alpha_{0}+\pi / 4}\left(A^{4} \sin ^{4}\left(\frac{\psi}{2}\right)+(B-A \sin \psi)^{2}\right)^{-s / 4} \mathrm{~d} \psi \\
& \lesssim \int_{\alpha_{0}}^{\alpha_{0}+\pi / 4}\left(A^{4} \sin ^{4} \psi+(B-A \sin \psi)^{2}\right)^{-s / 4} \mathrm{~d} \psi .
\end{aligned}
$$

Substituting $x=\sin \psi$ yields

$$
J_{s}(p, q) \lesssim \int_{x_{0}}^{x_{1}}\left(A^{4} x^{4}+(B-A x)^{2}\right)^{-s / 4} \frac{\mathrm{d} x}{\sqrt{1-x^{2}}}
$$

for some $x_{0}, x_{1} \in[0,1]$ satisfying

$$
\left|x_{0}-x_{1}\right| \leq \frac{\pi}{4}<1
$$

In view of (7.25) we conclude that either $x_{0} \geq \pi / 32$ or $x_{1} \leq 1-\pi / 32$ must be true. If $x_{0} \geq \pi / 32$, then

$$
J_{s}(p, q) \lesssim J_{1}:=\int_{0}^{1}\left(A^{4}+(B-A x)^{2}\right)^{-s / 4} \frac{\mathrm{d} x}{\sqrt{1-x}},
$$

while if $x_{1} \leq 1-\pi / 32$, then

$$
J_{s}(p, q) \lesssim J_{2}:=\int_{0}^{1}\left(A^{4} x^{4}+(B-A x)^{2}\right)^{-s / 4} \mathrm{~d} x .
$$

Our goal is to bound $J_{1}$ and $J_{2}$ from above by a multiple of $\left(A^{4}+B^{2}\right)^{-\sigma / 4}$. We accomplish this by considering various possibilities for the parameters $A$ and $B$. Throughout what follows, we make repeated use of the following elementary estimates. We omit the easy proofs, which proceed by splitting the integrals into pieces on which each respective factor in the integrand is dominating.

Lemma 7.8. Let $1<s<2$ and $\alpha, \delta \in[0,1]$. Then,

(i) if $\alpha+\delta \leq 1$, then $\int_{\alpha+\delta}^{1}(y-\alpha)^{-s / 2} \frac{\mathrm{d} y}{\sqrt{y}} \lesssim \max \left\{\delta, \frac{\alpha}{2}\right\}^{(1-s) / 2}$;

(ii) if $\alpha \leq \delta$ and $\delta-\alpha<1$, then $\int_{\delta-\alpha}^{1}(y+\alpha)^{-s / 2} \frac{\mathrm{d} y}{\sqrt{y}} \lesssim \delta^{(1-s) / 2}$;

(iii) if $\delta \leq \alpha$, then $\int_{0}^{\alpha-\delta}(\alpha-y)^{-s / 2} \frac{\mathrm{d} y}{\sqrt{y}} \lesssim \alpha^{-s / 2}(\alpha-\delta)^{1 / 2}$. 
Estimation of $J_{1}$. In this subsection, we discuss the integral

$$
J_{1}:=\int_{0}^{1}\left(A^{4}+(B-A x)^{2}\right)^{-s / 4} \frac{\mathrm{d} x}{\sqrt{1-x}} .
$$

We consider several possible cases depending on the relative sizes of the parameters $A$ and $B$.

If $2 A \leq B$ then the conclusion of Proposition 7.7 is trivially established. Indeed, in that case

$$
\begin{aligned}
J_{1} & \leq \int_{0}^{1}|B-A x|^{-s / 2} \frac{\mathrm{d} x}{\sqrt{1-x}} \lesssim B^{-s / 2} \int_{0}^{1} \frac{\mathrm{d} x}{\sqrt{1-x}} \\
& \lesssim B^{-s / 2} \lesssim\left(A^{4}+B^{2}\right)^{-s / 4} \lesssim d_{\mathbb{H}}(p, q)^{-\sigma}
\end{aligned}
$$

where we used the facts that $A^{4}+B^{2} \simeq B^{2}$ and $s<\sigma$.

For the remainder of this subsection, we assume that $B \leq 2 A$. We consider two cases.

Case 1: $B \leq A$.

Assume first that $2 A \leq 1-B / A$. We integrate over domains where each of the terms $A^{4}$ and $(B-A x)^{2}$ is dominating. Note that $|B-A x|=A^{2}$ if and only if $x=B / A \pm A$. In the present case, $B / A+A \leq 1$, however, $B / A-A$ can have any sign. We find that $J_{1}$ is comparable to

$$
\begin{aligned}
\int_{0}^{\max \left\{0, \frac{B}{A}-A\right\}}(B-A x)^{-s / 2} \frac{\mathrm{d} x}{\sqrt{1-x}} & +\int_{\max \left\{0, \frac{B}{A}-A\right\}}^{\frac{B}{A}+A} A^{-s} \frac{\mathrm{d} x}{\sqrt{1-x}} \\
& +\int_{\frac{B}{A}+A}^{1}(A x-B)^{-s / 2} \frac{\mathrm{d} x}{\sqrt{1-x}} .
\end{aligned}
$$

The second integral is $\lesssim A^{1-s}(1-B / A)^{-1 / 2}$. The first and third integrals can be estimated with the help of Lemma 7.8. Using parts (i) and (iii) of that lemma with $\alpha=1-B / A$ and $\delta=A$, we find that each of the remaining integrals is $\lesssim A^{-s / 2}(1-B / A)^{(1-s) / 2}$. Altogether, this yields

$$
J_{1} \lesssim A^{-s / 2}\left(1-\frac{B}{A}\right)^{(1-s) / 2}+A^{1-s}\left(1-\frac{B}{A}\right)^{-1 / 2} .
$$

Since $1-B / A \gtrsim A$, we conclude that

$$
J_{1} \lesssim A^{1 / 2-s}
$$

Now, suppose that $2 A \geq 1-B / A$. From the fact that $A<1 / 3$ it follows that $B / A-A \geq 0$, however, $B / A+A$ can be either greater or smaller than 1 . Similarly as in the previous case, we find that $J_{1}$ is comparable to

$$
\begin{aligned}
\int_{0}^{\frac{B}{A}-A}(B-A x)^{-s / 2} \frac{\mathrm{d} x}{\sqrt{1-x}} & +\int_{\frac{B}{A}-A}^{\min \left\{\frac{B}{A}+A, 1\right\}} A^{-s} \frac{\mathrm{d} x}{\sqrt{1-x}} \\
& +\int_{\min \left\{\frac{B}{A}+A, 1\right\}}^{1}(A x-B)^{-s / 2} \frac{\mathrm{d} x}{\sqrt{1-x}}
\end{aligned}
$$


The second integral is $\lesssim A^{-s}(1-B / A+A)^{1 / 2} \lesssim A^{1 / 2-s}$. Again, the first and third integrals can be estimated with the help of Lemma 7.8. Using parts (i) and (iii) of that lemma with $\alpha=1-B / A$ and $\delta=A$, we find that each of the remaining integrals is $\lesssim A^{1 / 2-s}$. Altogether, we conclude that

$$
J_{1} \lesssim A^{1 / 2-s} \text {. }
$$

Case 2: $A \leq B$.

First, suppose that $B / A-A \leq 1$. Note also that $B / A+A>1$ in this case. Similar computations as in the previous case yield

$$
\begin{aligned}
J_{1} & \simeq \int_{0}^{\frac{B}{A}-A}(B-A x)^{-s / 2} \frac{\mathrm{d} x}{\sqrt{1-x}}+\int_{\frac{B}{A}-A}^{1} A^{-s} \frac{\mathrm{d} x}{\sqrt{1-x}} \\
& \lesssim A^{1 / 2-s}+A^{-s}\left(1-\frac{B}{A}+A\right)^{1 / 2} .
\end{aligned}
$$

Here we have applied Lemma 7.8 (ii) with $\alpha=B / A-1$ and $\delta=A$ to the first integral. Since $B \geq A$ it follows that $1-B / A+A \leq A$ and hence

$$
J_{1} \lesssim A^{1 / 2-s}
$$

Finally, suppose that $B / A-A \geq 1$. Analogously to what was done as before, using Lemma 7.8 (ii) with $\alpha=\delta=B / A-1$, we obtain

$$
\begin{aligned}
J_{1} & \simeq \int_{0}^{1}(B-A x)^{-s / 2} \frac{\mathrm{d} x}{\sqrt{1-x}} \\
& =A^{-s / 2} \int_{0}^{1}\left(\frac{B}{A}-1+y\right)^{-s / 2} \frac{\mathrm{d} y}{\sqrt{y}} \lesssim A^{-s / 2}\left(\frac{B}{A}-1\right)^{(1-s) / 2} .
\end{aligned}
$$

Note that $B / A-1 \leq 1$ since $B \leq 2 A$. To complete the proof, we note that $B / A-1 \geq A$ in this case, whence

$$
J_{1} \lesssim A^{1 / 2-s} .
$$

By comparing (7.26), (7.27), (7.28), and (7.29) we see that in all cases $J_{1} \lesssim$ $A^{1 / 2-s}=A^{-\sigma / 2}$. Since we assumed $B \leq 2 A$ we have $A^{4}+B^{2} \lesssim A^{2}$ and so

$$
J_{1} \lesssim A^{-\sigma / 2} \lesssim\left(A^{4}+B^{2}\right)^{-\sigma / 4} \simeq d_{\mathbb{H}}(p, q)^{-\sigma}
$$

Estimation of $J_{2}$. We continue by examining the integral

$$
J_{2}=\int_{0}^{1}\left(A^{4} x^{4}+(B-A x)^{2}\right)^{-s / 4} \mathrm{~d} x .
$$

Again the idea is to integrate over intervals where one of the summands $A^{4} x^{4}$ and $(B-A x)^{2}$ is dominating. In order to see how these intervals should be chosen, we introduce the function

$$
f(x)=A^{2} x^{2}-|B-A x|
$$

and note that, for $x \in[0,1]$, we have $f(x)=0$ if and only if

$$
x=(2 A)^{-1}(\sqrt{1+4 B}-1) \quad \text { or } \quad x=(2 A)^{-1}(1-\sqrt{1-4 B}) .
$$


Since $B \leq 1 / 100$, we have that $B / A-2 B^{2} / A \leq(2 A)^{-1}(\sqrt{1+4 B}-1) \leq B / A$ and $B / A \leq(2 A)^{-1}(1-\sqrt{1-4 B}) \leq B / A+2 B^{2} / A$. If $x \in\left[0, B / A-2 B^{2} / \bar{A}\right]$ then $f(x)<0$ and so

$$
\left(A^{4} x^{4}+(B-A x)^{2}\right)^{-s / 4} \simeq(B-A x)^{-s / 2} .
$$

Next, suppose that $x \in\left[B / A-2 B^{2} / A, B / A+2 B^{2} / A\right]$. Since $B$ is small, we see that

$$
A^{2} x^{2} \geq B^{2}(1-2 B)^{2} \gtrsim B^{2} \gtrsim|B-A x|
$$

and so

$$
\left(A^{4} x^{4}+(B-A x)^{2}\right)^{-s / 4} \simeq A^{-s} x^{-s} .
$$

Finally, if $B / A+2 B^{2} / A<1$ and $x \in\left[B / A+2 B^{2} / A, 1\right]$ then again $f(x)<0$ and so

$$
\left(A^{4} x^{4}+(B-A x)^{2}\right)^{-s / 4} \simeq(A x-B)^{-s / 2} .
$$

As in the estimation of $J_{1}$, we may assume without loss of generality that $B \leq 2 A$.

Case 1: $B / A-2 B^{2} / A<1$.

Combining (7.30), (7.31) and (7.32), we see that

$$
\begin{aligned}
J_{2} \simeq \int_{0}^{\frac{B}{A}-\frac{2 B^{2}}{A}}(B-A x)^{-s / 2} \mathrm{~d} x & +\int_{\frac{B}{A}-\frac{2 B^{2}}{A}}^{\min \left\{\frac{B}{A}+\frac{2 B^{2}}{A}, 1\right\}} A^{-s} x^{-s} \mathrm{~d} x \\
& +\int_{\min \left\{\frac{B}{A}+\frac{2 B^{2}}{A}, 1\right\}}^{1}(A x-B)^{-s / 2} \mathrm{~d} x .
\end{aligned}
$$

These integrals can be evaluated exactly. We obtain

$$
\begin{aligned}
J_{2} \simeq & \frac{B^{1-s / 2}-\left(2 B^{2}\right)^{1-s / 2}}{A}+\frac{\left(\frac{B}{A}-\frac{2 B^{2}}{A}\right)^{1-s}-\left(\min \left\{\frac{B}{A}+\frac{2 B^{2}}{A}, 1\right\}\right)^{1-s}}{A^{s}} \\
& +\frac{(A-B)^{1-s / 2}-\left(2 B^{2}\right)^{1-s / 2}}{A} \cdot H\left(A-B-2 B^{2}\right),
\end{aligned}
$$

where $H(x)=1$ if $x>0$ and $H(x)=0$ if $x \leq 0$ is the Heaviside step function.

Applying the Mean Value Theorem to the second term on the right hand side of (7.33) and making a trivial estimate in the first and last terms gives

$$
\begin{aligned}
J_{2} & \lesssim \frac{B^{1-s / 2}}{A}+\frac{\left(\min \left\{\frac{B}{A}+\frac{2 B^{2}}{A}, 1\right\}-\left(\frac{B}{A}-\frac{2 B^{2}}{A}\right)\right) \xi^{-s}}{A^{s}}+\frac{(A-B)^{1-s / 2}}{A} \\
& \text { for some } \xi \in\left[\frac{B}{A}-\frac{2 B^{2}}{A}, \min \left\{\frac{B}{A}+\frac{2 B^{2}}{A}, 1\right\}\right] \\
& \lesssim \frac{B^{1-s / 2}}{A}+A^{-s} \frac{B^{2}}{A}\left(\frac{B}{A}-\frac{2 B^{2}}{A}\right)^{-s}+A^{-s / 2} .
\end{aligned}
$$

Since $B-2 B^{2}<A$ and $B$ is small, we have $B \lesssim A$ and so

$$
J_{2} \lesssim A^{-s / 2}+A^{-s} \frac{B^{2}}{A}\left(\frac{B}{A}-\frac{2 B^{2}}{A}\right)^{-s} \lesssim A^{-s / 2}+\frac{B^{2-s}}{A} \lesssim A^{-s / 2}+A^{1-s} \lesssim A^{-\sigma / 2}
$$


by the definition of $\sigma$ (see (7.21)). Since $B \leq 2 A$ we have $A^{4}+B^{2} \lesssim A^{2}$ and so

$$
J_{2} \lesssim\left(A^{4}+B^{2}\right)^{-\sigma / 4} \lesssim d_{\mathbb{H}}(p, q)^{-\sigma}
$$

as desired.

Case 2: $B / A-2 B^{2} / A \geq 1$.

In this case,

$$
J_{2} \simeq \int_{0}^{1}(B-A x)^{-s / 2} \mathrm{~d} x .
$$

Again we split the integral. For $x \in[0,1 / 2]$, we have $B-A x \geq B x$ (since $A \leq B$ in this case). For $x \in[1 / 2,1]$ we find that $B-A x \geq B(1-x)$. Hence

$$
\begin{aligned}
J_{2} & \simeq \int_{0}^{\frac{1}{2}} B^{-s / 2} x^{-s / 2} \mathrm{~d} x+\int_{\frac{1}{2}}^{1} B^{-s / 2}(1-x)^{-s / 2} \mathrm{~d} x \\
& \simeq B^{-s / 2} \leq B^{-\sigma / 2} \lesssim\left(A^{4}+B^{2}\right)^{-\sigma / 4} \lesssim d_{\mathbb{H}}(p, q)^{-\sigma} .
\end{aligned}
$$

With all the previous computations in hand, and recalling that $J_{s}(p, q)$ is bounded above by the maximum of $J_{1}$ and $J_{2}$, the proof of Proposition 7.7 is complete.

Remark 7.9. The result of Proposition 7.7 carries over without difficulty to arbitrary balls $B_{\mathbb{H}}\left(p_{0}, r\right)$ with $p_{0}=\left(z_{0}, t_{0}\right)$ and $r<\frac{1}{20}\left|z_{0}\right|$.

We use Proposition 7.7 to prove the following theorem on the almost sure dimensions of vertical projections of subsets of vertical planes.

Theorem 7.10. Let $A$ be a Borel set of dimension $\operatorname{dim}_{\mathbb{H}} A>1$ lying inside a vertical plane $\mathbb{W}_{\theta_{0}}$. Then, for almost every $\theta \in[0, \pi)$,

$$
\operatorname{dim}_{\mathbb{H}} p_{\mathbb{W}_{\theta}}(A) \geq \frac{\operatorname{dim}_{\mathbb{H}} A}{2}+\frac{1}{2} .
$$

Proof. As $A$ lies inside a vertical plane, its Hausdorff dimension with respect to $d_{\mathbb{H}}$ can be at most 3 . In Proposition 7.7 an estimate for $J_{s}(p, q)$ was established for points $p$ and $q$ in the intersection of a particular ball with $\mathbb{W}_{\theta_{0}}$, cf. Remark 7.9. One can argue similarly as in the proof of Proposition 7.5 and assume without loss of generality that $A$ lies inside such a ball.

Since $\operatorname{dim}_{\mathbb{H}} A>1$, there exists $\sigma \in\left(1, \operatorname{dim}_{\mathbb{H}} A\right)$ and $\mu \in \mathcal{M}(A)$ so that $I_{\sigma}(\mu)<\infty$. Let $s:=\sigma / 2+1 / 2$. Then $1<s<2$. By Proposition 7.7, we see that $J_{s}(p, q)$ is bounded above by a multiple of $d_{\mathbb{H}}(p, q)^{-\sigma}$, whence

$$
\begin{aligned}
\int_{0}^{\pi} I_{s}\left(\left(p_{\mathbb{W}_{\theta}}\right)_{\sharp}(\mu)\right) \mathrm{d} \theta & =\int_{A} \int_{A} J_{s}(p, q) \mathrm{d} \mu(p) \mathrm{d} \mu(q) \\
& \lesssim \int_{A} \int_{A} d_{\mathbb{H}}(p, q)^{-\sigma} \mathrm{d} \mu(p) \mathrm{d} \mu(q)=I_{\sigma}(\mu)
\end{aligned}
$$


which is finite. Thus $\operatorname{dim}_{\mathbb{H}} p_{\mathbb{W}_{\theta}}(A) \geq s$ for almost every $\theta$. Letting $\sigma$ increase to $\operatorname{dim}_{\mathbb{H}} A$ yields

$$
\operatorname{dim}_{\mathbb{H}} p_{\mathbb{W}_{\theta}}(A) \geq \frac{\operatorname{dim}_{\mathbb{H}} A}{2}+\frac{1}{2} \text { for almost every } \theta .
$$

This completes the proof of the theorem.

\subsection{Further examples}

We give some additional examples related to the theorems about projection onto vertical subspaces $\mathbb{W}_{\theta}$.

Example 7.11. We give an example of a set where the lower bound for the projections is known but cannot be derived by the energy method in the usual way. Let

$$
A=\left\{\left(x, x^{2}\right): x \in[0,2]\right\} .
$$

We will prove the following: for any $s<\operatorname{dim}_{\mathbb{H}} A=2$ and $\mu \in \mathcal{M}(A)$ with $I_{s}(\mu)<\infty$

$$
\int_{0}^{\pi} I_{3 / 2}\left(\left(p_{\mathbb{W}_{\theta}}\right)_{\sharp} \mu\right) \mathrm{d} \theta=\infty .
$$

However,

$$
\operatorname{dim}_{\mathbb{H}} p_{\mathbb{W}_{\theta}}(A)=2 \text { for all but one } \theta \in[0, \pi) .
$$

To see why (7.36) holds, observe that

$$
p_{\mathbb{W}_{\theta}}\left(x, x^{2}\right)=\left\{\left(-x \sin \theta \mathbf{i} e^{\mathbf{i} \theta},(1+\sin 2 \theta) x^{2}\right): x \in[1,2]\right\} .
$$

Hence, $p_{\mathbb{W}_{\theta}}(A)$ is a parabola for all $\theta \in[0, \pi)$ except $\theta=3 \pi / 4$, in which case it is a horizontal line segment. One can easily verify that the sets $p_{\mathbb{W}_{\theta}}(A)$ for $\theta \neq 3 \pi / 4$ are non horizontal and hence have dimension two.

Now let $s<\operatorname{dim}_{\mathbb{H}} A$ and take $\mu \in \mathcal{M}(A)$ with $I_{s}(\mu)<\infty$. Such a measure exists by the energy version of Frostman's lemma. By the Fubini-Tonelli theorem and the integration formula for pushforward measures, we find

$$
\int_{0}^{\pi} I_{3 / 2}\left(\left(p_{\mathbb{W}_{\theta}}\right)_{\sharp} \mu\right) \mathrm{d} \theta=\int_{A} \int_{A} \int_{0}^{\pi} d_{\mathbb{H}}\left(p_{\mathbb{W}_{\theta}}(p), p_{\mathbb{W}_{\theta}}(q)\right)^{-3 / 2} \mathrm{~d} \theta \mathrm{d} \mu(p) \mathrm{d} \mu(q) .
$$

We will now estimate the integral $K=\int_{0}^{\pi} d_{\mathbb{H}}\left(p_{\mathbb{W}_{\theta}}(p), p_{\mathbb{W}_{\theta}}(q)\right)^{-3 / 2} \mathrm{~d} \theta$ for arbitrary points $p=\left(x, x^{2}\right)$ and $q=\left(y, y^{2}\right)$ in $A$. For such points, we find

$$
d_{\mathbb{H}}\left(p_{\mathbb{W}_{\theta}}(p), p_{\mathbb{W}_{\theta}}(q)\right)=\left((y-x)^{4} \sin ^{4} \theta+(1+\sin 2 \theta)^{2}\left(x^{2}-y^{2}\right)^{2}\right)^{1 / 4}
$$

Since $x, y \in[1,2]$, we have that $\left|x^{2}-y^{2}\right| \leq 4|x-y|$ and therefore,

$$
d_{\mathbb{H}}\left(p_{\mathbb{W}_{\theta}}(p), p_{\mathbb{W}_{\theta}}(q)\right) \leq 2\left(a^{4} \sin ^{4} \theta+a^{2}(1+\sin (2 \theta))^{2}\right)^{1 / 4},
$$

where $a=|x-y|$. 
Then,

$$
\begin{aligned}
K & \gtrsim \int_{0}^{\pi}\left(a^{4} \sin ^{4} \theta+a^{2}(1+\sin (2 \theta))^{2}\right)^{-3 / 8} \mathrm{~d} \theta \\
& =\int_{0}^{2 \pi}\left(a^{4} \sin ^{4}\left(\frac{\psi}{2}\right)+a^{2}(1-\sin \psi)^{2}\right)^{-3 / 8} \mathrm{~d} \psi \\
& \geq \int_{0}^{\pi / 2}\left(a^{4} \sin ^{4}\left(\frac{\psi}{2}\right)+a^{2}(1-\sin \psi)^{2}\right)^{-3 / 8} \mathrm{~d} \psi \\
& \gtrsim \int_{0}^{\pi / 2}\left(a^{4} \sin ^{4} \psi+a^{2}(1-\sin \psi)^{2}\right)^{-3 / 8} \mathrm{~d} \psi,
\end{aligned}
$$

where we used the fact that $0 \leq \sin (\psi / 2) \leq \sin \psi$ for $\psi \in[0, \pi / 2]$. Substituting $\sin \psi=x$ yields

$$
K \gtrsim \int_{0}^{1}\left(a^{4} x^{4}+a^{2}(1-x)^{2}\right)^{-3 / 8} \frac{\mathrm{d} x}{\sqrt{1-x}} .
$$

Further elementary computations yield

$$
K \gtrsim \int_{1-a}^{1}\left(a^{4}+a^{2}(1-x)^{2}\right)^{-3 / 8} \frac{\mathrm{d} x}{\sqrt{1-x}} \gtrsim a^{-3 / 2} \int_{1-a}^{1} \frac{\mathrm{d} x}{\sqrt{1-x}} \gtrsim a^{-1} .
$$

In order to compare this to the distance between $p$ and $q$, we observe that

$$
d_{\mathbb{H}}(p, q)=\left((x-y)^{4}+\left(x^{2}-y^{2}\right)^{2}\right)^{1 / 4} \gtrsim a^{1 / 2}
$$

and thus

$$
K \gtrsim d_{\mathbb{H}}(p, q)^{-2}
$$

Therefore,

$$
\int_{0}^{\pi} I_{3 / 2}\left(\left(p_{\mathbb{W}_{\theta}}\right)_{\sharp} \mu\right) \mathrm{d} \theta \gtrsim I_{2}(\mu) .
$$

Since $A$ has finite Hausdorff 2-measure, $I_{2}(\mu)$ is infinite for all $\mu \in \mathcal{M}(A)$. Hence (7.35) holds.

Remark 7.12. More generally, take a subset $A$ of the parabola in (7.34) with $\operatorname{dim}_{\mathbb{H}} A>0$ and $0<s<\operatorname{dim}_{\mathbb{H}} A$. There exists $\mu \in \mathcal{M}(A)$ such that $I_{s}(\mu)<\infty$, but $I_{u}(\mu)=\infty$ for all $u>s$.

Using Proposition 7.7, it can be proved that $\int_{0}^{\pi} I_{\sigma}\left(\left(p_{\mathbb{W}_{\theta}}\right)_{\sharp} \mu\right) \mathrm{d} \theta$ is finite provided that $\sigma \leq s / 2+1 / 2$. One could hope that even if we cannot get a pointwise estimate as in Proposition 7.7 for $\sigma>s / 2+1 / 2$, nevertheless the set of points where this bound does not hold might be small with respect to $\mu$ so that we could still apply the energy method as usual to derive $\frac{1}{2}\left(\operatorname{dim}_{\mathbb{H}} A+1\right)$ as an almost sure lower bound for the vertical projections.

However, a reasoning analogous to that in the preceding example yields that for all $\sigma>s / 2+1 / 2$,

$$
\int_{0}^{\pi} I_{\sigma}\left(\left(p_{\mathbb{W}_{\theta}}\right)_{\sharp} \mu\right) \mathrm{d} \theta \gtrsim I_{2 \sigma-1}(\mu)=\infty .
$$

This shows that the energy method cannot be applied in the usual way to improve the lower bound which was obtained in Theorem 7.10. 
As we have shown earlier, the estimate

$$
J_{s}(p, q) \lesssim d_{\mathbb{H}}(p, q)^{-s}
$$

holds for $p$ and $q$ in an arbitrary set $A \subset \mathbb{H}$ when $s<1$. In Proposition 7.2 an analogous estimate was obtained for larger $s$ under the additional assumption that $A$ is contained in a horizontal plane. The following example shows that one cannot hope to obtain (7.37) for an arbitrary set and $s \geq 1$.

Example 7.13. There exist points $p, q \in B_{\mathbb{H}}\left((1,0), \frac{1}{20}\right)$ so that $J_{s}(p, q)=\infty$ for all $s>1$.

Choose $p=(z, t)$ and $q=(\zeta, \tau)$ with $\left|z^{2}-\zeta^{2}\right|=t-\tau>0$ and $\varphi_{2}-\varphi_{1}=\pi / 2$, where $\varphi_{1}$ and $\varphi_{2}$ are defined in (7.5). Denoting $a=\left|z^{2}-\zeta^{2}\right|=t-\tau$, we find that

$$
\begin{aligned}
J_{s}(p, q) & \gtrsim \int_{0}^{\pi}\left(a^{4} \sin ^{4}\left(\varphi_{1}-\theta\right)+a^{2}\left(1-\sin \left(\varphi_{1}+\varphi_{2}-2 \theta\right)\right)^{2}\right)^{-s / 4} \mathrm{~d} \theta \\
& \simeq \int_{0}^{\pi}\left(a^{4} \sin ^{4}\left(\frac{\psi}{2}\right)+a^{2}(1-\cos \psi)^{2}\right)^{-s / 4} \mathrm{~d} \psi \\
& \gtrsim \int_{0}^{\pi / 2}\left(a^{4} \sin ^{4}\left(\frac{\psi}{2}\right)+a^{2}(1-\cos (\psi))^{2}\right)^{-s / 4} \mathrm{~d} \psi \\
& \simeq \int_{0}^{\pi / 2}\left(a^{4} \sin ^{4} \psi+a^{2}(1-\cos (\psi))^{2}\right)^{-s / 4} \mathrm{~d} \psi
\end{aligned}
$$

The substitution $x=\sin \psi$ leads to

$$
J_{s}(p, q) \gtrsim \int_{0}^{1}\left(a^{4} x^{4}+a^{2}\left(1-\sqrt{1-x^{2}}\right)^{2}\right)^{-s / 4} \mathrm{~d} x .
$$

For $x \in[0,1]$ one has the estimate $1-\sqrt{1-x^{2}} \lesssim x^{2}$, whence

$$
J_{s}(p, q) \gtrsim\left(a^{4}+a^{2}\right)^{-s / 4} \int_{0}^{1} x^{-s} \mathrm{~d} x=\infty \quad \text { whenever } s>1 .
$$

\section{Final remarks}

In this final section, we present concluding remarks and suggestions for future work.

\subsection{Sharpness of the lower bound for vertical projections}

While we know that our upper dimension bound for vertical projections is sharp, this need not be the case for the lower bound. It could be a subject of further research to study this lower bound. Our belief that the current lower bound might not be sharp is motivated by the partial results which we obtain under additional assumptions on the regularity or the shape of the set $A$. If the lower bound given in Theorem 1.4 is still optimal, examples to prove the sharpness should be sought among less regular, fractal-type sets which are not entirely contained in a horizontal or vertical plane. 


\subsection{Projection of horizontal sets}

In order to better understand the behavior of the vertical projections, it might be enlightening to study the effect of these mappings on the dimension of so-called horizontal subsets $A$ of $\mathbb{H}$ which realize equality in the dimension comparison statement $(2.3)$. In the low codimensional case $\left(\operatorname{dim}_{\mathbb{H}} A \geq 2\right)$ horizontal sets have been constructed as invariant sets for self-similar iterated function systems. An example of such a set is the so-called Heisenberg square A, a self-similar subset of $\mathbb{H}$ first considered by Strichartz. According to [22] and [3], the Hausdorff dimension of $A$ is equal to two, in fact,

$$
0<\mathcal{H}_{\mathbb{H}}^{2}(A)<\infty
$$

and, by Theorem 1.14 in [3], every $\pi$-section of $A$ coincides with the graph of a special function of bounded variation $(\mathrm{SBV})$ on $Q=[0,1]^{2}$. Let us fix an angle $\theta \in[0, \pi)$. In order to better understand the effect of the vertical projection $p_{\mathbb{W}_{\theta}}$ on the Heisenberg square, we slice the set $A$ with planes that are orthogonal (in the Euclidean sense) to $\mathbb{W}_{\theta}$ and study the images of these slices under the projection.

To this end, we identify the horizontal subgroup $\mathbb{V}_{\theta}$ with a linear subspace of $\mathbb{R}^{2}$. We denote by $Q_{\theta}$ the orthogonal projection of $Q$ to the orthocomplement $\mathbb{W}_{\theta} \cap\{t=0\}$ of $\mathbb{V}_{\theta}$ and we denote by $Q_{a}^{\theta}$ the set of points $(r+\mathbf{i} a) e^{\mathbf{i} \theta}$ in $Q$ which project to $\mathbf{i} a e^{\mathbf{i} \theta}$ in the orthocomplement. Note that $Q_{a}^{\theta}$ is either empty, or a singleton, or corresponds to a one-dimensional interval via the identification $(r+\mathbf{i} a) e^{\mathbf{i} \theta} \leftrightarrow r$. We write $u_{a}^{\theta}$ for the function

$$
u_{a}^{\theta}(r)=u\left((r+\mathbf{i} a) e^{\mathbf{i} \theta}\right)
$$

defined on $Q_{a}^{\theta}$. By Theorems 3.107 and 3.108 of [1],

$$
u_{a}^{\theta} \in \mathrm{SBV} \text { for } \mathcal{H}^{1} \text {-a.e. } a \text {. }
$$

Let $G_{a}^{\theta}$ denote the graph of the restriction of $u$ to $Q_{a}^{\theta}$. Using (4.1) we find that

$$
p_{\mathbb{W}_{\theta}}\left(G_{a}^{\theta}\right)=\left\{\left(\mathbf{i} a e^{\mathbf{i} \theta}, u_{a}^{\theta}(r)-2 a r\right):(r+\mathbf{i} a) e^{\mathbf{i} \theta} \in Q_{a}^{\theta}\right\} .
$$

It might be interesting to study the size of the set $p_{\mathbb{W}_{\theta}}\left(G_{a}^{\theta}\right)$, or, equivalently, of

$$
\left\{u_{a}^{\theta}(r)-2 a r: r \in Q_{a}^{\theta}\right\},
$$

considered as a subset of a vertical line. This might be useful to estimate or compute the Hausdorff dimension of $p_{\mathbb{W}_{\theta}}(A)$, similarly to what was done in the proof of Proposition 6.1.

\subsection{Exceptional sets}

Associated to every almost sure dimension theorem is a corresponding problem concerning exceptional sets. Theorem 2.3 asserts that, for a Borel set $A \subset \mathbb{R}^{n}$, the exceptional set

$$
\left\{V \in G(n, m): \operatorname{dim} P_{V}(A)<\min \{\operatorname{dim} A, m\}\right\}
$$


has zero $\gamma_{n, m}$-measure. It is natural to ask whether the size of this exceptional set can be controlled in a quantitative fashion. Namely, when $\sigma<\min \{\operatorname{dim} A, m\}$, how large can the Hausdorff dimension of the exceptional set $\{V \in G(n, m)$ : $\left.\operatorname{dim} P_{V}(A) \leq \sigma\right\}$ be? This question was answered by Kaufman and Mattila [13], [14], [17]; see also Falconer [7] and Peres-Schlag [21]. For simplicity we restrict to the case $n=2, m=1$. Let $A \subset \mathbb{R}^{2}$ be Borel. Kaufman [13] showed that the set of lines $L \in G(2,1)$ for which $\operatorname{dim} P_{L}(A)<s=\operatorname{dim} A$ has $\mathcal{H}^{s}$ measure zero when $s<1$. Falconer [7] gave complementary results for $\operatorname{dim} A>1$, one of which asserts that the set of lines $L \in G(2,1)$ for which $\operatorname{dim} P_{L}(A)<\sigma \leq \operatorname{dim} A$ has dimension bounded above by $1+\sigma-\operatorname{dim} A$. Coupling this with Lemma 3.1 gives preliminary information about the size of exceptional sets for the dimensions of horizontal projections in the Heisenberg group.

Proposition 8.1. Let $A \subset \mathbb{H}$ be a Borel set with $3<\operatorname{dim}_{\mathbb{H}} A \leq 4$. For each $2<\sigma \leq \operatorname{dim}_{\mathbb{H}} A$,

$$
\operatorname{dim}\left(\left\{\theta: \operatorname{dim} p_{\mathbb{V}_{\theta}}(A)<\sigma-2\right\}\right) \leq 1+\sigma-\operatorname{dim}_{\mathbb{H}} A .
$$

The proof is quite easy. By Lemma 3.1, $\operatorname{dim}_{E} \pi(A) \geq \operatorname{dim}_{\mathbb{H}} A-2$. Applying the aforementioned result of Falconer yields

$$
\begin{aligned}
\operatorname{dim}\left(\left\{\theta: \operatorname{dim} p_{\mathbb{V}_{\theta}}(A)<\sigma-2\right\}\right) & \leq 1+(\sigma-2)-\operatorname{dim}_{E} \pi(A) \\
& \leq 1+(\sigma-2)-\left(\operatorname{dim}_{\mathbb{H}} A-2\right)=1+\sigma-\operatorname{dim}_{\mathbb{H}} A,
\end{aligned}
$$

as desired.

Other problems related to exceptional sets could be posed. In particular, it would be interesting to obtain some estimates on the size of exceptional sets associated to the almost sure dimension statements in Theorem 1.4.

\subsection{Higher dimensional Heisenberg groups}

We anticipate that the results of this paper extend to the Heisenberg groups $\mathbb{H}^{n}=$ $\mathbb{C}^{n} \times \mathbb{R}=\mathbb{R}^{2 n+1}$ of arbitrary dimension. We expect similar results to hold for the semidirect splitting of $\mathbb{H}^{n}$ into any horizontal subgroup of dimension $m, 1 \leq m \leq n$, and its complementary vertical subgroup. The horizontal subgroup can be identitified with a subspace of the base space $\mathbb{R}^{2 n}$. Note however that not every member of the Grassmanian $G(2 n, m)$ generates a horizontal subgroup of $\mathbb{H}^{n}$. The correct class of subspaces to consider are the isotropic subspaces of dimension $m$ inside $\mathbb{R}^{2 n}$, equipped with its standard symplectic structure. The resulting isotropic Grassmanian is a submanifold of $G(2 n, m)$ whose dimension is $m(2 n-m)-\left(\begin{array}{c}m \\ 2\end{array}\right)$. It can be equipped with a natural measure, either as the appropriate Hausdorff measure in this dimension for the metric obtained by restricting the metric from $G(2 n, m)$, or by a direct construction similar to that of the measures $\gamma_{n, m}$ on $G(n, m)$. This construction provides a natural framework to investigate dimensions of projections in the Heisenberg groups of arbitrary dimension. We plan to return to this topic in a later paper. ${ }^{1}$

\footnotetext{
${ }^{1}$ Note added in print: some of the results of the present paper have recently been generalized in [2] to higher dimensional Heisenberg groups.
} 


\section{References}

[1] Ambrosio, L., Fusco, N. And Pallara, D.: Functions of bounded variation and free discontinuity problems. Oxford Mathematical Monographs, The Clarendon Press Oxford University Press, New York, 2000.

[2] Balogh, Z. M., Fässler, K., Mattila, P. And Tyson, J. T.: Projection and slicing theorems in Heisenberg groups. Adv. Math. 231 (2012), no. 2, 569-604.

[3] Balogh, Z. M., Höfer-Isenegger, R. and Tyson, J. T.: Lifts of Lipschitz maps and horizontal fractals in the Heisenberg group. Ergod. Theory Dynam. Syst. 26 (2006), no. 3, 621-651.

[4] Balogh, Z. M., Rickly, M. and Serra-Cassano, F.: Comparison of Hausdorff measures with respect to the Euclidean and Heisenberg metric. Publ. Mat. 47 (2003), no. 1, 237-259.

[5] Balogh, Z. M. And Tyson, J. T.: Hausdorff dimensions of self-similar and selfaffine fractals in the Heisenberg group. Proc. London Math. Soc. (3) 91 (2005), no. $1,153-183$.

[6] Balogh, Z. M., Tyson, J. T. and Warhurst, B.: Sub-Riemannian vs. Euclidean dimension comparison and fractal geometry on Carnot groups. Adv. Math. 220 (2009), no. 2, 560-619.

[7] FAlconer, K. J.: Hausdorff dimension and the exceptional set of projections. Mathematika 29 (1982), no. 1, 109-115.

[8] Falconer, K. J.: Fractal geometry. Mathematical Foundations and Applications, John Wiley \& Sons, Chichester, 1990.

[9] Franchi, B., Serapioni, R. and Serra-Cassano, F.: Intrinsic Lipschitz graphs in Heisenberg groups. J. Nonlinear Convex Anal. 7 (2006), no. 3, 423-441.

[10] Franchi, B., Serapioni, R. and Serra-Cassano, F.: Differentiability of intrinsic Lipschitz functions within Heisenberg groups. J. Geom. Anal. 21 (2011), no. 4, $1050-6926$.

[11] Gromov, M.: Carnot-Carathéodory spaces seen from within. In Sub-Riemannian Geometry, 79-323. Progress in Mathematics 144, Birkhäuser, Basel, 1996.

[12] Howroyd, J. D.: On dimension and on the existence of sets of finite positive Hausdorff measure. Proc. London Math. Soc. (3) 70 (1995), no. 3, 581-604.

[13] Kaufman, R.: On Hausdorff dimension of projections. Mathematika 15 (1968), 153-155.

[14] Kaufman, R. And Mattila, P.: Hausdorff dimension and exceptional sets of linear transformations. Ann. Acad. Sci. Fenn. Ser. A I Math. 1 (1975), no. 2, 387-392.

[15] Magnani, V.: Contact equations, Lipschitz extensions and isoperimetric inequalities. Calc. Var. Partial Differential Equations 39 (2010), no. 1-2, 233-271.

[16] Marstrand, J. M.: Some fundamental geometrical properties of plane sets of fractional dimensions. Proc. London Math. Soc. (3) 4 (1954), 257-302.

[17] Mattila, P.: Hausdorff dimension, orthogonal projections and intersections with planes. Ann. Acad. Sci. Fenn. Ser. A I Math. 1 (1975), no. 2, 227-244.

[18] Mattila, P.: Geometry of sets and measures in Euclidean spaces. Cambridge Studies in Advanced Mathematics 44, Cambridge University Press, Cambridge, 1995.

[19] Mattila, P.: Hausdorff dimension, projections, and the Fourier transform. Publ. Mat. 48 (2004), no. 1, 3-48. 
[20] Mattila, P., Serapioni, R. and Serra-Cassano, F.: Characterizations of intrinsic rectifiability in Heisenberg groups. Ann. Sc. Norm. Super. Pisa Cl. Sci. (5) 9 (2010), no. 4, 687-723.

[21] Peres, Y. and Schlag, W.: Smoothness of projections, Bernoulli convolutions, and the dimension of exceptions. Duke Math. J. 102 (2000), no. 2, 193-251.

[22] Strichartz, R. S.: Self-similarity on nilpotent Lie groups. In Geometric analysis (Philadelphia, PA, 1991), 123-157. Contemp. Math. 140, Amer. Math. Soc., Providence, RI, 1992.

Received May 25, 2011.

Zoltán M. BAlogh: Mathematisches Institut, Universität Bern, Sidlerstrasse 5, 3012 Bern, Switzerland.

E-mail: zoltan.balogh@math.unibe.ch

Estibalitz Durand-Cartagena: Departamento de Matemática Aplicada, UnED, C/ Juan del Rosal 12, 28040-Madrid, Spain.

E-mail: edurand@ind.uned.es

KATRIN Fässler: Matematiikan ja tilastotieteen laitos, Gustaf Hällströmin katu 2b, 00014 Helsingin Yliopisto, Finland.

E-mail: katrin.fassler@helsinki.fi

Pertti Mattila: Matematiikan ja tilastotieteen laitos, Gustaf Hällströmin katu 2b, 00014 Helsingin Yliopisto, Finland.

E-mail: pertti.mattila@helsinki.fi

Jeremy T. Tyson: Department of Mathematics, University of Illinois at UrbanaChampaign, 1409 West Green St., Urbana, IL 61801, USA.

E-mail: tyson@math.uiuc.edu

ZMB and KF supported by the Swiss National Science Foundation, European Science Foundation Project HCAA and the FP7 EU Commission Project CG-DICE. EDC supported in part by DGES (Spain) Project MTM2009-07848 and by Grant AP2006-00620. PM supported by Academy of Finland grants 135463 and 1141137. JTT supported by NSF Grant DMS-0901620. 